\title{
Direct numerical simulation of turbulent flow in a circular pipe subjected to radial system rotation
}

by

\section{Zhao-Ping Zhang}

\author{
A thesis \\ presented to the University of Manitoba \\ in fulfilment of the \\ thesis requirement for the degree of \\ Master of Science \\ in \\ Mechanical Engineering
}

Winnipeg, Manitoba, Canada 2019

(C)Zhao-Ping Zhang 2019 


\begin{abstract}
In this thesis, direct numerical simulations have been preformed with a high-order spectral element method computer code to investigate the Coriolis force effect on a fully-developed turbulent flow confined within a circular pipe subjected to radial system rotations. In order to study the radially rotating effects on the flow, a wide range of rotation numbers $\left(R o_{\tau}\right)$ have been tested. In response to the system rotation imposed, large-scale secondary flows appear as streamwise counter-rotating vortices, which highly interact with the boundary layer and have a significant impact on the turbulent flow structures and dynamics. A quasi Taylor-Proudman region occurs at low rotation numbers, where the mean axial velocity is invariant along the rotating axis. As the rotation number increases, laminarization occurs near the bottom wall of the pipe, and the flow become fully laminarized when the rotation number approaches $R o_{\tau}=1.0$. The characteristics of the flow field are investigated in both physical and spectral spaces, which include the analyses of the first- and second-order statistical moments, pre-multiplied spectra of velocity fluctuations, budget balance of the transport equation of Reynolds stresses, and coherent flow structures.
\end{abstract}




\section{Acknowledgements}

I would like to express my great gratitude to my supervisor Prof. Bing-Chen Wang for his encouragement, support and guidance, and also for his experience and suggestions in social communication which was very helpful to me on how to conduct myself during my M.Sc. study in Canada.

I would also like to express my appreciation to Dr. Xing-Jun Fang for his generous help and guidance on my M.Sc. study and project during his PhD program.

I am so grateful to all my lab mates for their academic advices and help during my M.Sc. study, and also for the friendly working and studying environment they created in the lab.

My special thanks goes to Prof. M. F. Tachie and Prof. S. J. Ormiston for their help and suggestions during my course study.

I am also thankful to my friends that I made in Canada, and I enjoyed the time and moment that spent with them during my M.Sc. program.

Last but not the least, I would like to express my sincere gratitude to my parents, Shan-Gui Zhang and Xiao-Ning Liu for their unconditional love, support and encouragement. 


\section{Contents}

Abstract $\quad$ i

$\begin{array}{ll}\text { Acknowledgements } & \text { ii }\end{array}$

List of Figures $\quad$ vi

Nomenclature $\quad x$

1 Introduction $\quad 1$

1.1 Background ........................... 1

1.2 Literature review . . . . . . . . . . . . . . . . . . 1

1.2.1 Turbulent 2D-plane channel and square duct flows subjected to spanwise system rotation . . . . . . . . . . . . 2

1.2.2 Turbulent circular pipe flows subjected to radial system rotation 3

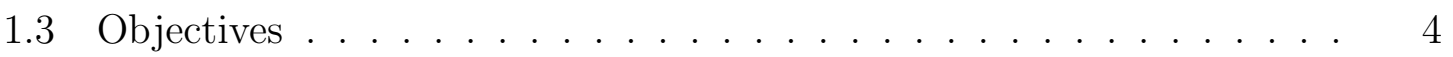

1.4 Thesis Outline ......................... 4

2 Test Case and Numerical Algorithm 5 
3 Results analysis $\quad 9$

3.1 Test of the Axial Domain Size . . . . . . . . . . . . . . . . . . 9

3.2 Instantaneous flow . . . . . . . . . . . . . . . . . 15

3.3 Mean flow . . . . . . . . . . . . . . . . . . . . . . . . 17

3.4 Turbulence statistics _. . . . . . . . . . . . . . . . . . 22

3.5 Turbulence structures . . . . . . . . . . . . . . . . . . . 34

4 Conclusions and future studies $\quad 39$

4.1 Conclusions on DNS of turbulent flow in a circular pipe subjected to radial system rotation . . . . . . . . . . . . . . . 39

4.2 Future studies . . . . . . . . . . . . . . . . . . . . . . . . 42

$\begin{array}{ll}\text { References } & 48\end{array}$

A Spectral-Element Method $\quad 49$

A.1 Time-splitting Algorithm . . . . . . . . . . . . . . 50

A.2 Spatial discretization . . . . . . . . . . . . . . 52

A.2.1 Weighted residual method . . . . . . . . . . . . 52

A.2.2 Discretization in a $1 \mathrm{D}$ quadrilateral element . . . . . . . 54

A.2.3 Discretization in a 2D quadrilateral element . . . . . . . . 56

A.2.4 Global operations . . . . . . . . . . . . . . . 59

A.3 Linear solver. . . . . . . . . . . . . . . . . . . . . . . 60

A.3.1 Iterative solver for the viscous substep . . . . . . . . . . . 61

A.3.2 Direct solver for the pressure substep . . . . . . . . . . 62 
B Transport equations of Reynolds stresses 


\section{List of Figures}

2.1 Configuration of a circular pipe under radial system rotation in both cylindrical and rectangular coordinate systems. The angular speed of radial system rotation of the pipe is $\Omega$. The three Coriolis force components are denoted using $C_{r}, C_{\theta}$ and $C_{z}$ (in the radial, azimuthal and axial directions, respectively), with the arrows indicating their directions. . . . . . . . . . . . . . . . . . . .

2.2 Cross-sectional view of the mesh. A spectral-element method is used to discretize the cross section of the pipe with 352 quadrilateral-structural elements with each element constructed using an 8th-order GaussLobatto-Legendre (GLL) Lagrange polynomial. In the axial direction (not shown), Fourier expansion of 960 modes is used for spatial dis-

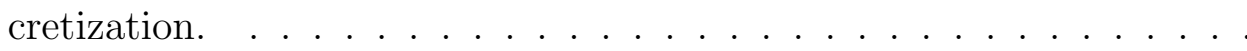

3.1 Comparison of turbulence intensities as a function of $y^{+}$in the central vertical plane $\left(\theta=90^{\circ}\right)$ and two-point auto-correlation coefficients along the axial direction of the pipe at the wall-normal position of $y^{+}=14.24$ (on the pressure side of the pipe, for $\theta=90^{\circ}$ ) for $L_{z}=2 \pi R$, $5 \pi R, 8 \pi R, 12 \pi R$ and $20 \pi R$. The comparison is made with the rotation number kept at $R o_{\tau}=0.2 \ldots \ldots \ldots \ldots \ldots \ldots$ 
3.2 Pre-multiplied energy spectra for different computational domain sizes $L_{z}$ at $R o_{\tau}=0.2$. The vertical dashed lines in panel (a) demarcate the computational domain sizes of $L_{z}=2 \pi R, 5 \pi R, 8 \pi R, 12 \pi R$, and $20 \pi R$, respectively. The isopleths in panel (b) are plotted with respect to wavelength $\lambda_{z}^{+}$and wall coordinate $y^{+}$. The cross symbol "+" in panel (b) indicates the location $\left(y^{+}=12.99, \lambda_{z}^{+}=1407.83\right)$ of the peak value of $k_{z} \phi_{z z}$. The value of the inner and outer isopleths is 1.92 and 0.82 (corresponding to $70 \%$ and $30 \%$ of the peak value of $k_{z} \phi_{z z}$, respectively). The pre-multiplied energy spectra have been non-

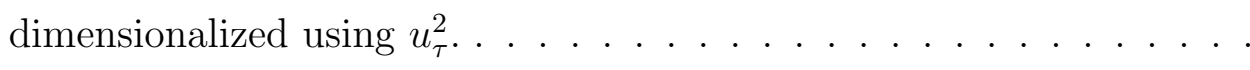

3.3 Contours of the instantaneous axial velocity $u_{z}^{+}$in a cross-stream plane at four different rotation numbers. . . . . . . . . . . . . .

3.4 Contours of the mean axial velocity $\left\langle u_{z}\right\rangle^{+}$superimposed with mean streamlines in the cross-stream plane at different rotation numbers. .

3.5 Comparison of bulk mean velocity $U_{b}^{+}$, volume-averaged TKE $k_{m}^{+}$, and skin friction coefficient $C_{f}$ as a function of the rotation number. The TKE value has been non-dimensionalized using $u_{\tau}^{2}$. . . . . . . . . .

3.6 Profiles of mean axial velocity $\left\langle u_{z}\right\rangle^{+}$at six different rotation numbers. Arrow points to the direction of monotonic variation of the rotation

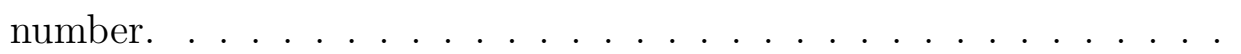

3.7 Contours of TKE $k^{+}$in a cross-stream plane at four different rotation numbers. The contour legends are kept identical in panels (a) and (b) in order to facilitate a direct comparison of TKE levels at four rotation numbers. Furthermore, contours corresponding to low TKE levels (for $\left.k^{+} \leq 0.7\right)$ are clipped to highlight turbulent regions. The TKE value has been non-dimensionalized using $u_{\tau}^{2}$. . . . . . . . . . . . . . 
3.8 Profiles of Reynolds stresses in the central vertical plane $\left(\theta=90^{\circ}\right)$ of the pipe at different rotation numbers. Arrow points to the direction of an increasing rotation number. . . . . . . . . . . . . . .

3.9 Effect of radial system rotation on the pre-multiplied velocity spectra calculated along the axial line of the pipe at wall-normal position $y^{+}=$ 14.24 on the pressure side $\left(\theta=90^{\circ}\right)$ of the pipe. Arrow points to the direction of an increasing rotation number. The pre-multiplied energy spectra $k_{z} \phi_{i i}$ (no summation convention implied here) have been nondimensionalized using $u_{\tau}^{2}$. . . . . . . . . . . . . . . . . . . . . . . .

3.10 Profiles of the budget terms of $\left\langle u_{z}^{\prime} u_{z}^{\prime}\right\rangle$ in the central vertical plane $\left(\theta=90^{\circ}\right)$ of the pipe for different rotation numbers. All budget terms have been non-dimensionalized using $u_{\tau}^{3} / R$. . . . . . . . . . . .

3.11 Profiles of the budget terms of $\left\langle u_{r}^{\prime} u_{r}^{\prime}\right\rangle$ in the central vertical plane $\left(\theta=90^{\circ}\right)$ of the pipe for different rotation numbers. All budget terms have been non-dimensionalized using $u_{\tau}^{3} / R \ldots \ldots \ldots$

3.12 Profiles of the budget terms of $\left\langle u_{z}^{\prime} u_{r}^{\prime}\right\rangle$ in the central vertical plane $\left(\theta=90^{\circ}\right)$ of the pipe for different rotation numbers. All budget terms have been non-dimensionalized using $u_{\tau}^{3} / R$. . . . . . . . . . .

3.13 Pre-multiplied spectra of the budget terms of $\left\langle u_{z}^{\prime} u_{z}^{\prime}\right\rangle$ with respect to wavelength $\lambda_{z}^{+}$calculated along the axial line of the pipe at wall-normal position $y^{+}=14.24$ on the pressure side $\left(\theta=90^{\circ}\right)$ for $R_{\tau}=0.0,0.2$ and 0.8. All budget terms have been non-dimensionalized using $u_{\tau}^{3} / R$.

3.14 Contours of instantaneous axial vorticity fluctuation $\omega_{z}^{\prime+}$ in a cross-

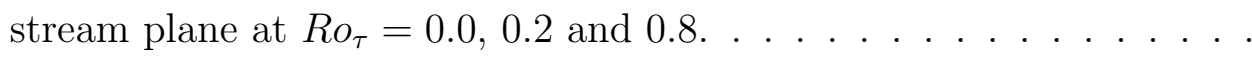


3.15 Contours of vortical structures for the non-rotating $\left(R o_{\tau}=0.0\right)$ and rotating $\left(R o_{\tau}=0.2\right.$ and 0.8$)$ cases. Only one-fourth axial and onehalf cross-stream domain (iso-surfaces are plotted with $\lambda_{c i}=0.8$ ) is displayed. The contours are colored with non-dimensionalized radius

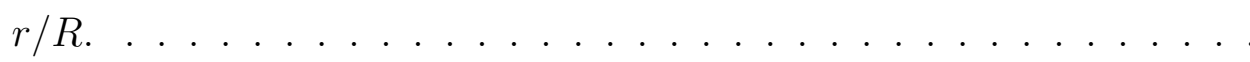

3.16 Contours of streamwise-elongated Taylor-Görtler vortical structures for the rotating pipe flow $\left(R o_{\tau}=0.8\right)$, based on time-averaged axial vorticity fluctuations ${\overline{\omega_{z}^{\prime}}}^{+}$. Only one-fourth of pipe length (iso-surfaces are plotted with $\left.\left|{\overline{\omega_{z}^{\prime}}}^{+}\right|=0.7\right)$ is displayed. Red and blue colours represent negative and positive values of ${\overline{\omega_{z}^{\prime}}}^{+}$, respectively. The two partially enlarged panels are extracted to demonstrate the characteristic instantaneous turbulent flow structures in the cross-stream direction, superimposed with time-averaged velocity vectors. . . . . . . . . 


\section{Nomenclature}

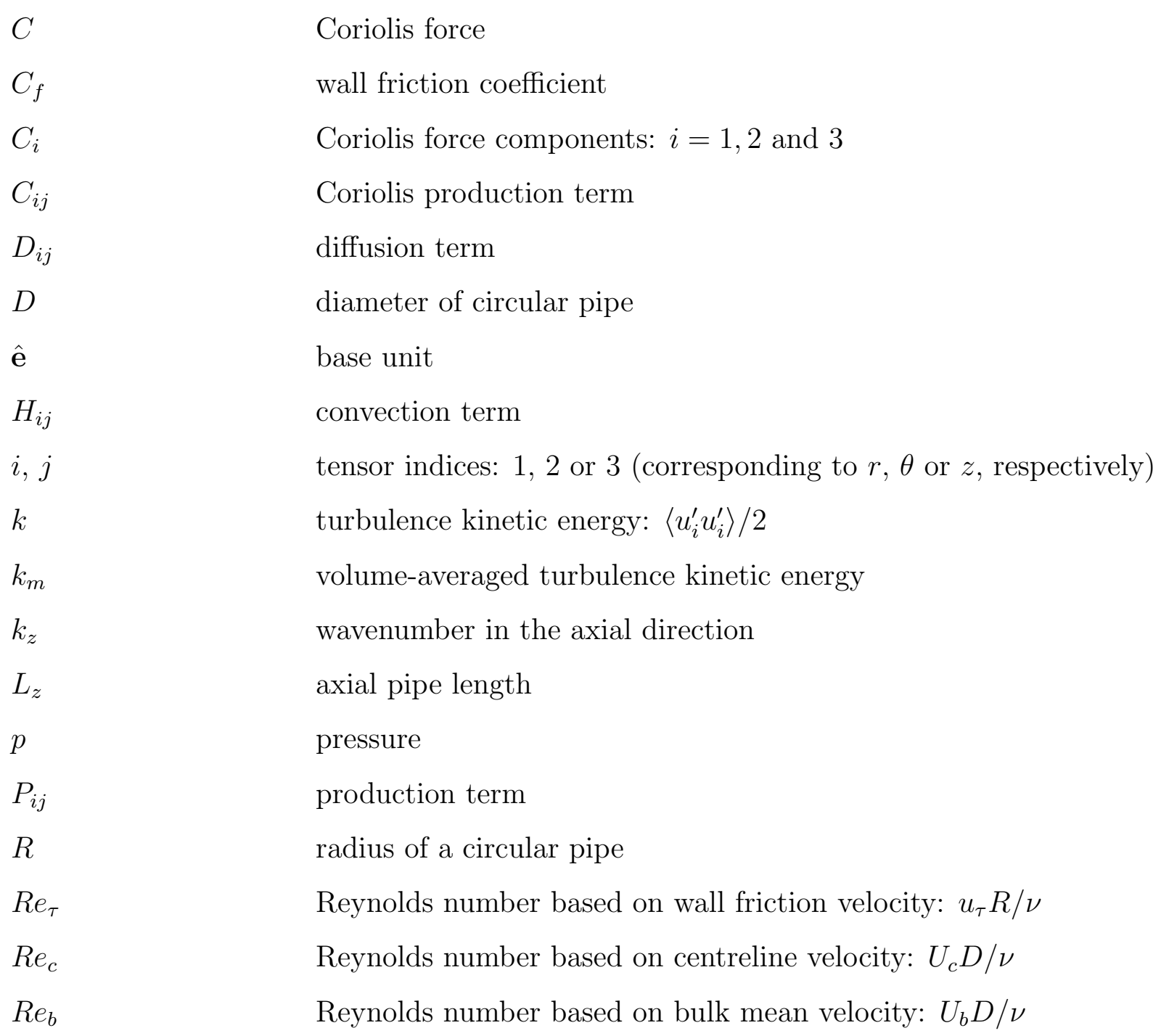




$\begin{array}{ll}R o_{\tau} & \text { rotation number: } 2 \Omega R / u_{\tau} \\ R_{i i} & \text { two-point auto-correlation coefficients for velocity } \\ & \text { fluctuations } u_{i}^{\prime}: i=1,2 \text { and } 3 \\ t & \text { time } \\ u & \text { velocity } \\ u_{i} & \text { velocity components: } i=1,2 \text { and } 3 \\ u_{\tau} & \text { wall friction velocity } \\ U_{b} & \text { bulk mean velocity } \\ U_{c} & \text { centreline velocity } \\ r, \theta, z & \text { radial, azimuthal and axial coordinates, respectively } \\ x, y, z & \text { horizontal, vertical and axial coordinates, respectively }\end{array}$

\section{Greek Letters}

$\begin{array}{ll}\varepsilon_{i j} & \text { dissipation term } \\ \lambda_{c i} & \text { swirling strength } \\ \nu & \text { kinematic viscosity } \\ \Omega & \text { angular velocity of the pipe } \\ \omega_{z} & \text { axial vorticity: }\left[\partial\left(r u_{\theta}\right) / \partial r-\partial u_{r} / \partial \theta\right] / 2 r \\ \Pi & \text { mean streamwise pressure gradient } \\ \Pi_{i j} & \text { pressure-strain term } \\ \rho & \text { density } \\ \tau_{w} & \text { wall shear stress } \\ \phi_{i j} & \text { velocity spectra } \\ \nabla & \text { del operator }\end{array}$




\section{Subscripts}
$(\cdot)_{r},(\cdot)_{\theta},(\cdot)_{z}$
radial, azimuthal and axial components, respectively
$(\cdot)_{i j}$
second-order tensors: $i, j=1,2$ and 3

\section{Superscripts}
$(\cdot)^{t}$
value at the top wall
$(\cdot)^{b}$
value at the bottom wall
$(\cdot)^{s}$
value at the lateral side wall
$\overline{(\cdot)}$
$(\overrightarrow{\cdot)}$
time-averaging
vector
$(\cdot)^{+}$
wall coordinates
$\langle\cdot\rangle$
time- and streamwise-averaging

\section{Miscellaneous}

$\begin{array}{ll}\text { DNS } & \text { direct numerical simulation } \\ \text { DOF } & \text { degree of freedom } \\ \text { FD } & \text { finite difference } \\ \text { FV } & \text { finite volume } \\ \text { GLL } & \text { Gauss-Lobatto-Legendre } \\ \text { IBP } & \text { integration by part } \\ \text { LDA } & \text { laser Doppler anemometer } \\ \text { LES } & \text { large-eddy simulation }\end{array}$




$\begin{array}{ll}\text { LETOT } & \text { large-eddy turnover time: } R / u_{\tau} \\ \text { MPI } & \text { message passing interface } \\ \text { PCG } & \text { preconditioned conjugate gradient } \\ \text { RANS } & \text { Reynolds-averaged Navier-Stokes } \\ \text { RMS } & \text { root-mean-square } \\ \text { SEM } & \text { spectral element method } \\ \text { TFI } & \text { Transfinite Interpolation } \\ \text { TGL } & \text { Taylor-Görtler-like } \\ \text { TKE } & \text { turbulence kinetic energy } \\ \text { TP } & \text { Taylor-Proudman } \\ \text { TS } & \text { Tollmien-Schlichting } \\ \text { VLSM } & \text { very large-scale motions }\end{array}$




\section{Chapter 1}

\section{Introduction}

\subsection{Background}

Turbulent flows confined within a circular pipe subjected to radial system rotation (the rotating direction being perpendicular to the axial center of the pipe) represent an important subject with engineering applications in rotary machinery, rotating heat exchangers and centrifugal separators. In response to the radial system rotation, Coriolis forces act on the fluid flows as body forces, inducing large-scale secondary flows in the cross-stream direction. The appearance of large-scale secondary flows has a significant impact on the flow physics, statistical moments, and coherent structures.

\subsection{Literature review}

In this section, previous studies of the rotating effect on the turbulent boundary flows are reviewed, which include the turbulent spanwise-rotating 2D-plane channel and square duct flows, and turbulent radially-rotating circular pipe flows. 


\subsubsection{Turbulent 2D-plane channel and square duct flows sub- jected to spanwise system rotation}

In the current literature, numerical and experimental studies of rotating flows have primarily focused on spanwise-rotating flows confined within either plane channels or square ducts. Belhoucine et al. [1] conducted Reynolds averaged Navier-Stokes (RANS) simulations of spanwise-rotating duct flows based on several explicit algebraic Reynolds stress models. They observed that the cross-stream flows consisted of two large and two small counter-rotating vortices at a sufficiently high rotation number. Kristoffersen and Andersson [2] conducted direct numerical simulations (DNS) to investigate spanwise-rotating plane channel flows. They observed Taylor-Görtler-like vortices in the pattern of counter-rotating streamwise roll cells in the cross-stream direction, and laminarization of the flow on the suction side of the plane channel at high rotation numbers. Grundestam et al. [3] investigated the occurrence of laminarization in a spanwise-rotating plane channel, and indicated that the large-scale longitudinal vortices in a spanwise-rotating channel are analogous to the TaylorGörtler vortices induced by surface curvature. Wallin et al. [4] also performed DNS of spanwise-rotating channel flows using a pseudo-spectral method code. Through a linear stability analysis, they showed that full laminarization is dominated by the cycle of Tollmien-Schlichting (TS) waves at sufficiently high rotation numbers. Recently, Xia et al. [5] conducted DNS to investigate the spanwise-rotating effects on the first- and second-order statistical moments of the velocity field. Pallares and Davidson [6] conducted large-eddy simulation (LES) to study the turbulent flow confined within a square duct at different rotation numbers. They investigated the effects of system rotation on the secondary flows and the interaction of flow structures with the four sidewalls. They demonstrated that system rotation significantly affects the secondary flows which appear as counter-rotating streamwise roll cells near the cor- 
ners of duct, and descends the turbulence level associated with flow stabilization and the development of a Taylor-Proudman (TP) region at high rotation numbers. Dai et al. [7] conducted DNS to investigate the effects of spanwise system rotation on the secondary flow pattern confined within a square duct. More specifically, they studied the influence of the Coriolis force on the secondary flow of Prandtl's second kind and formation of vortical flow structures. Recently, Fang et al. [8] systematically studied the spanwise-rotating square duct flows using DNS, and observed that complete laminarization is almost reached near the top and side walls of the square duct at sufficiently high rotation numbers.

\subsubsection{Turbulent circular pipe flows subjected to radial sys- tem rotation}

Turbulent flow through a stationary (non-rotating) circular pipe is a classical subject and has been well studied using DNS (Eggels et al. [9], Wu and Moin [10], Chin et al. [11], and $\mathrm{Wu}$ et al. [12]). However, based on a thorough literature review, it is noticed that detailed studies of radially-rotating circular pipe are still lacking, and the underlying flow physics of the impact of the Coriolis force on the generation of both mean and turbulent secondary flow structures are not yet well understood. In his pioneering work, Barua [13] studied radially-rotating laminar pipe flows using an analytical approach, and obtained a semi-empirical equation to estimate the skin friction coefficient. Ishigaki $[14,15]$ conducted DNS to analyze the similarity between curved pipe flows and radially-rotating pipe flows. Both laminar and turbulent flow patterns were investigated, and Ishgaki $[14,15]$ was able to demonstrate a similarity between these two types of flows with respect to the skin friction coefficient, mean Nusselt number and secondary flow structures. Besides a limited number of numerical studies of radially-rotating pipe flow, the number of experimental studies is also very 
limited in the current literature. Lei et al. [16] performed a series of laser Doppler anemometry (LDA) experiments of fully-developed laminar pipe flows under radial system rotation. They studied the effects of Coriolis force on the mean and secondary flows under different Reynolds numbers and rotation numbers.

\section{$1.3 \quad$ Objectives}

The objective of this thesis is to conduct a systematic DNS study of turbulent circular pipe flows under radial system rotation for a wide range of rotation numbers. In total, 11 rotation numbers are tested, ranging from $R o_{\tau}=0.0$ to 1.0. The characteristics of the flow field are compared against those of a non-rotating pipe flow, and the effects of the Coriolis force on the mean and turbulent flow structures are examined in both physical and spectral spaces. Furthermore, through a budget analysis of Reynolds stresses transport equations, the rotating effect on the turbulence energy transfer between velocity fluctuating components are investigated.

\subsection{Thesis Outline}

The remainder of this thesis is organized as follows: in Chapter 2, the test case and numerical algorithm for solving the governing equations will be introduced. In Chapter 3, the DNS results will be analyzed to understand the first- and secondorder statistical moments of the velocity field, spectra of velocity fluctuations, and budget balance of the transport equation of Reynolds shear stresses. In Chapter 4, major findings of this research and future studies will be summarized. Finally, in Appendix A, the algorithm of the spectral-element code is documented. 


\section{Chapter 2}

\section{Test Case and Numerical}

\section{Algorithm}

Figure 2.1 describes the circular pipe configuration with respect to both cylindrical and rectangular coordinate systems. For the cylindrical coordinate system, $r, \theta$ and $z$ represent the radial, azimuthal and axial coordinates, and the three corresponding velocity components are denoted using $u_{r}, u_{\theta}$ and $u_{z}$, respectively. The axial-length of the circular pipe is set to $L_{z}=20 \pi R$, where $R$ is the pipe radius. In order to study the rotating effects, a wide range of rotation numbers $\left(R o_{\tau}=2 \Omega R / u_{\tau}\right)$ have been tested, varying from $R o_{\tau}=0.0$ (corresponding to the non-rotating case) to 1.0 (corresponding to the full laminarization case), with the Reynolds number fixed at $R e_{\tau}=u_{\tau} R / \nu=180$. Here, $\nu$ is the kinematic viscosity of the fluid. The flow is fully developed, with a periodic boundary condition applied to the axial direction and a no-slip condition enforced on the curved pipe surface. The equations that govern an 


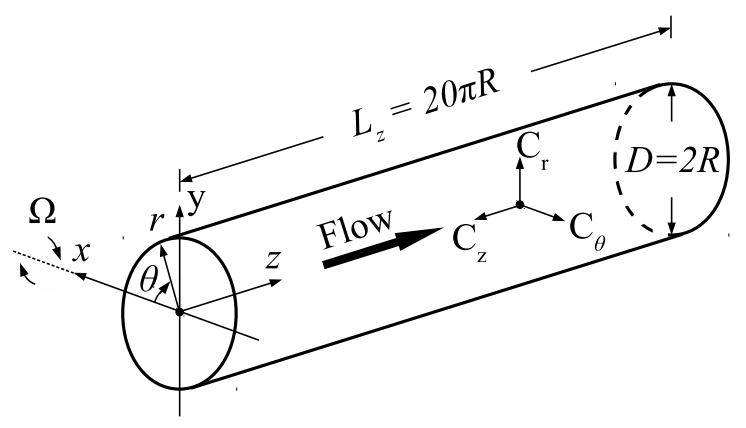

Figure 2.1: Configuration of a circular pipe under radial system rotation in both cylindrical and rectangular coordinate systems. The angular speed of radial system rotation of the pipe is $\Omega$. The three Coriolis force components are denoted using $C_{r}, C_{\theta}$ and $C_{z}$ (in the radial, azimuthal and axial directions, respectively), with the arrows indicating their directions.

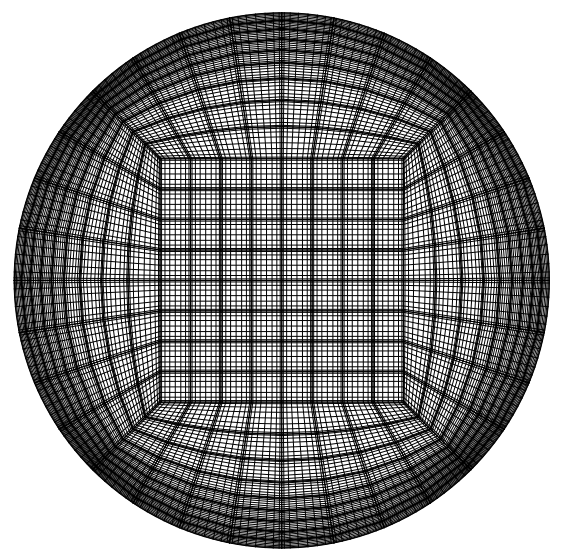

Figure 2.2: Cross-sectional view of the mesh. A spectral-element method is used to discretize the cross section of the pipe with 352 quadrilateral-structural elements with each element constructed using a 8th-order Gauss-Lobatto-Legendre (GLL) Lagrange polynomial. In the axial direction (not shown), Fourier expansion of 960 modes is used for spatial discretization.

incompressible flow with respect to a radially-rotating reference frame read

$$
\begin{gathered}
\nabla \cdot \vec{u}=0 \\
\frac{\partial \vec{u}}{\partial t}+\vec{u} \cdot \nabla \vec{u}=-\Pi \hat{\mathbf{e}}_{z}-\frac{1}{\rho} \nabla p+\nu \nabla^{2} \vec{u}+\vec{C},
\end{gathered}
$$

where $\vec{u}, \rho$ and $p$ represent the velocity, density and pressure of the fluid, respectively, $\Pi$ is the constant mean axial pressure gradient, and $\hat{\mathbf{e}}_{z}$ is the base unit vector of the $z$-direction, with $\left|\hat{\mathbf{e}}_{z}\right| \equiv 1$. As shown in Fig. 2.1, the pipe rotates counterclockwise (about the positive $x$-direction) at a constant angular speed $\Omega$. In response to the system rotation, Coriolis force $\vec{C}$ appears, with three components: $C_{r}=2 \Omega u_{z} \sin \theta$, $C_{\theta}=2 \Omega u_{z} \cos \theta$ and $C_{z}=-2 \Omega\left(u_{r} \sin \theta+u_{\theta} \cos \theta\right)$ in the radial, azimuthal and axial directions, respectively. 
The simulations were conducted with a spectral-element code so-called "Semtex" (Blackburn and Sherwin [17]) developed using the $\mathrm{C}++$ and FORTRAN programming languages, and parallelized following the message passing interface (MPI) standard. Figure 2.2 provides a cross-sectional view of the mesh used for performing DNS of the pipe flow. This mesh is generated to not only satisfy the near-wall refinement, but also to avoid the singularity problem at the pipe center in the discretization of the governing equations with respect to a cylindrical coordinate system. Similar meshes were used in Karniadakis and Sherwin [18], Sharma and Nandakumar [19], El Khoury et al. [20], Di Liberto et al. [21] and Bolis et al. [22] for numerical simulation of non-rotating pipe flows. This mesh was divided in 352 quadrilateral structural elements, and a 8th-order Gauss-Lobatto-Legendre (GLL) Lagrange polynomial was used for spatial discretization of each element. All physical quantities were calculated with a Fourier expansion of 960 modes along the axial direction. In total, 21.8 million grid points were used to perform the DNS. The grid resolution, measured in wall coordinates, is uniform in the axial direction with $\Delta z^{+}=11.78$, and varies in the azimuthal and radial directions with $R \Delta \theta^{+}=1.78-6.40, \Delta r^{+}=0.25$ at the first node off the wall and $\Delta r^{+}=1.03$ at the pipe center. It should be indicated here that in comparison with a finite-volume code (which is typically second-order accurate), the Semtex code of Blackburn and Sherwin [17] used for performing the current DNS is based on a high-accuracy spectral-element algorithm [17, 18], free from any nonphysical numerical dissipation $[8,23]$. In effect, the current axial and azimuthal grid resolutions are comparable to those used by Eggels et al. [9], Wu and Moin [10], Wagner et al. [24] and Chin et al. [25] in their DNS studies of turbulent non-rotating circular pipe flows at similar Reynolds numbers. However, the radial grid resolution of the current DNS is mush finer than those implemented in these classical DNS papers. The maximum radial grid resolution is $\Delta r_{\max }^{+}=1.03$ in the current study, but was kept at $\Delta r_{\max }^{+}=1.647-4.32$ in Eggels et al. [9], Wu and Moin [10], Wagner et 
al. [24] and Chin et al. [25]. All simulations were performed on the WestGrid (Western Canada Research Grid) supercomputers. In order to compute the flow statistics, for each simulation at each rotation number, 300 instantaneous flow fields were generated with approximately $310 \mathrm{~GB}$ data over 80 large-eddy turnover times (LETOTs, defined as $\left.R / u_{\tau}\right)$.

In result analysis, an instantaneous turbulence variable $\Phi$ is decomposed as $\Phi=$ $\langle\Phi\rangle+\Phi^{\prime}$, where $\langle\Phi\rangle$ is an averaged value over time and the homogeneous $(z)$ direction, and $\Phi^{\prime}$ denotes the relative fluctuating value. All quantities expressed in wall coordinates are indicated using superscript ' + ', and the nominal wall friction velocity $u_{\tau}$ used for calculating the wall coordinates is determined based on the non-rotating pipe flow case. The origin for the radial coordinates $r$ is located at the pipe center. In order to conduct near-wall analysis (as in a turbulent plane channel), a wall coordinate $y^{+}$is also introduced to simplify the symbol system, which is defined as $y^{+} \stackrel{\text { def }}{=}(R-r) u_{\tau} / \nu$. 


\section{Chapter 3}

\section{Results analysis}

In this chapter, the DNS results of radially-rotating pipe flow are analyzed and compared against those of a non-rotating pipe flow. The comparative study includes 11 rotation numbers, ranging from $R o_{\tau}=0.0$ to 1.0. In the following (sections 3.1-3.5), I first investigate the circular pipe length effects on turbulence statistics with turbulence intensities, two-point auto-correlation coefficients and pre-multiplied energy spectra; and then present a study of the instantaneous and mean velocity fields to explain the basic physical characteristics of the flow, followed by the analyses of statistical moments, spectra of velocity fluctuations, budget balance of the transport equation of Reynolds stresses, and turbulent flow structures.

\subsection{Test of the Axial Domain Size}

In DNS of a fully-developed turbulent pipe flow under a periodic boundary condition applied to the axial direction, the only geometrical factor that needs to be considered is the axial length of the computational domain, which has a direct impact on the predictive accuracy of flow statistics. Eggels et al. [9] conducted experiments 
and DNS to study fully-developed pipe flows at Reynolds number $R e_{\tau}=180$ and compared their pipe flow results with those of a fully-developed plane channel flow. In their study, an axial-length of $10 R$ was used to perform numerical simulations which led to nonzero correlation of velocity fluctuations at half axial length of the computational domain, indicating that this pipe length was insufficient for producing accurate flow statistics. Later, Kim and Adrian [26] conducted wind-tunnel measurements using hot-film anemometry, and investigated the spectra of the axial velocity fluctuations. They observed very large-scale motions (VLSM) in the outer layer region, with a maximal wavelength of $12 R-14 R$. Recently, Chin et al. [11] conducted DNS of fully developed turbulent pipe flows at Reynolds numbers of $R e_{\tau}=170$ and 500 to investigate the axial domain size effects on turbulence statistics. Based on a thorough examination of the turbulence intensities, two-point correlations and the spectra of velocity fluctuations, they suggested that an axial domain size of $8 \pi R$ was enough to capture all mean and turbulent flow structures at $R e_{\tau}=170$. In their DNS study of stationary low-Reynolds number turbulent plane channel flow, Kim et al. [27] indicated that the streamwise domain size should be kept long enough in order to capture the largest scale eddy motions by ensuring that the velocity fluctuations become uncorrelated over one half the streamwise domain length. However, based on the study of Chin et al. [11], it is clear that the criterion for judging a proper axial domain size for a pipe flow can be based on many physical quantities other than just the two-point auto-correlation of velocity fluctuations. Recently, Yang and Wang [28] performed DNS of streamwise-rotating plane channel flows at very high rotation numbers in order to investigate the influence of the Coriolis force on the streamwise growth of the Taylor-Görtler vortices. They studied the effect of streamwise domain size on the predictive accuracy of flow statistics in both physical and spectral spaces, and concluded that the minimal computational domain depends strongly on the specific physical quantity under investigation. 
Thus far, a systematic study of the proper axial computational domain size for numerical simulation of radially-rotating pipe flow is still lacking in the current literature. In view of this, I aim at performing a comparative study to choose a proper streamwise computational domain size for conducting DNS of circular pipe flows under radial system rotations. Following the approach of Yang and Wang [28] for DNS of a streamwise-rotating plane channel flow, I will judge the effect of the axial pipe dimension on the predictive accuracy of a variety of physical quantities such as turbulence intensities, two-point auto-correlation coefficients, and pre-multiplied energy spectra of velocity fluctuations. Five pipe lengths for $L_{z}=2 \pi R, 5 \pi R, 8 \pi R, 12 \pi R$ and $20 \pi R$ will be compared. The longest pipe (i.e., $L_{z}=20 \pi R$ ) tested here is also the longest in the current literature on DNS study of radially-rotating pipe flows. In the following analysis, I will focus on the comparison of a rotating flow of $R o_{\tau}=0.2$ against a non-rotating flow (of $R o_{\tau}=0.0$ ). The reason that DNS results of $R o_{\tau}=0.2$ are presented here is that the highest turbulence level is observed on the pressure side of the pipe at this particular rotation number. In fact, it will be shown later that at the highest rotation number $\left(R o_{\tau}=1.0\right)$ tested in this research, the pipe flow actually becomes fully laminarized.

Figure 3.1(a) compares the root-mean square (RMS) values of the three velocity components of the rotating flow obtained based on five different pipe lengths in the central vertical plane $\left(\theta=90^{\circ}\right)$ at $R o_{\tau}=0.2$. Clearly, owing to the system rotation imposed, the profiles of all three RMS velocities are no longer symmetrical across the central vertical plane. The minimal values of turbulence intensities occur at $y^{+}=108.41$, a location that deviates from the pipe center (at $y^{+}=180$ ). It is interesting to observe that the peak value of $u_{z, r m s}^{\prime+}$ is well predicted in all five cases of different computational pipe lengths. The peak value of $u_{z, r m s}^{++}$is around 2.82 at $y^{+}=14.24$ on the pressure side $\left(\theta=90^{\circ}\right)$ of the pipe and is around 2.21 at $y^{+}=338.65$ on the suction side $\left(\theta=270^{\circ}\right)$ of the pipe. Furthermore, as is clear 


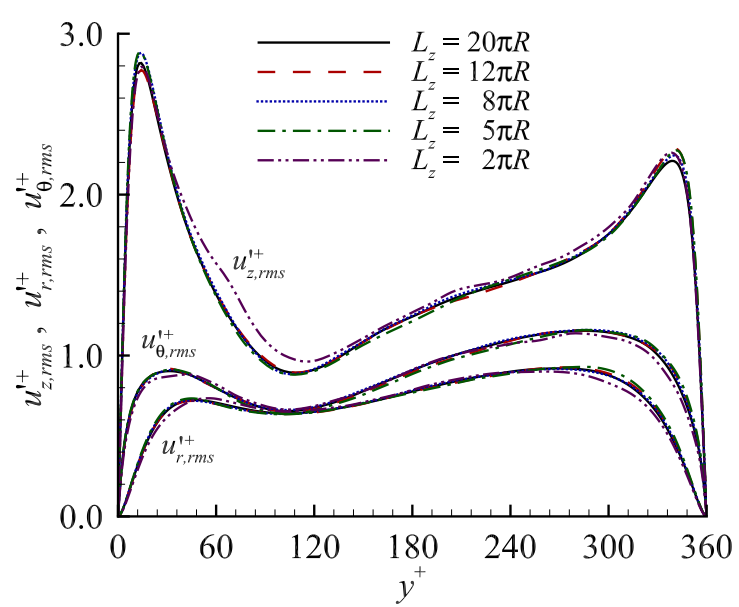

(a) Turbulence intensity

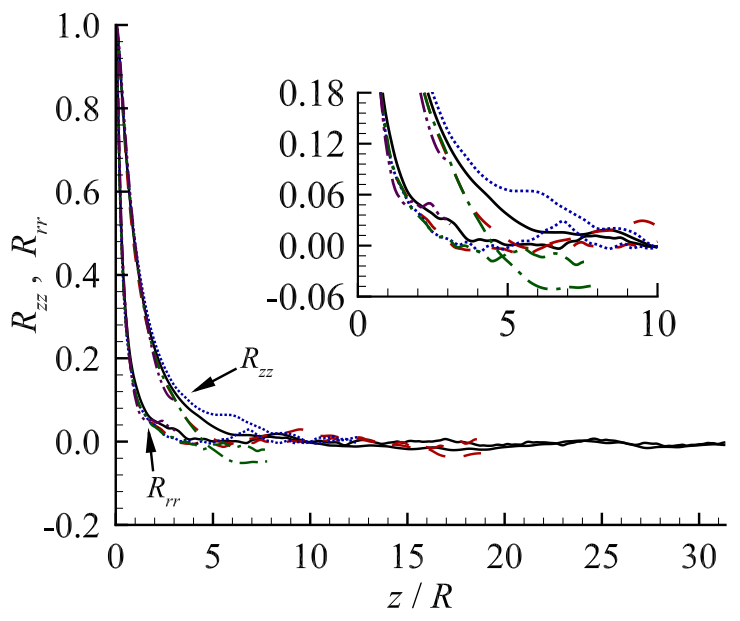

(b) Two point auto-correlation coefficients

Figure 3.1: Comparison of turbulence intensities as a function of $y^{+}$in the central vertical plane $\left(\theta=90^{\circ}\right)$ and two-point auto-correlation coefficients along the axial direction of the pipe at the wall-normal position of $y^{+}=14.24$ (on the pressure side of the pipe, for $\theta=90^{\circ}$ ) for $L_{z}=2 \pi R, 5 \pi R, 8 \pi R, 12 \pi R$ and $20 \pi R$. The comparison is made with the rotation number kept at $R o_{\tau}=0.2$.

from Fig. 3.1(a), the value of $u_{r, r m s}^{\prime+}$ and $u_{\theta, r m s}^{\prime}$ are insensitive to the pipe lengths testes. However, the profiles of $u_{z, r m s}^{+}$show that the predictions based on three longer pipes ( $L_{z}=8 \pi R, 12 \pi R$ and $20 \pi R$ ) are close to each other. In comparison, the value of $u_{z, r m s}^{\prime+}$ predicted based on the pipe length of $L_{z}=2 \pi R$ is slightly higher in the pipe center. These indicate that the relatively short computational pipe lengths of $L_{z}=2 \pi R$ and $5 \pi R$ are insufficient to give an accurate prediction of the $u_{z, r m s}^{\prime+}$ value. This observation is consistent with the study of non-rotating pipe flows of Chin et al. [11]. From Fig. 3.1(a), it is observed that the value of turbulence intensity $u_{z, r m s}^{\prime+}$ reaches it maximum at wall-normal position $y^{+}=14.24$ on the pressure side of the rotating pipe. In view of this, this specific position has been chosen for the calculation of the two-point auto-correlation coefficients and pre-multiplied energy spectra in my comparative study of the pipe length.

Figure 3.1(b) compares the two-point auto-correlation coefficients along the axial 
direction of the pipe at the wall-normal position $y^{+}=14.24$ for five different computational domain sizes at $R o_{\tau}=0.2$. In the figure, $R_{z z}$ and $R_{r r}$ represent the axial and radial two-point correlations, respectively. As is evident in Fig. 3.1(b), the value of $R_{r r}$ drops towards zero rather quickly as the pipe length increases. However, the tend of $R_{z z}$ behaves in a different manner than does $R_{r r}$ in the sense that the value of $R_{z z}$ falls more slowly than that of $R_{r r}$. From Fig. 3.1(b), it is clear that the value of the $R_{z z}$ and $R_{r r}$ for three longer pipe lengths $\left(L_{z}=8 \pi R, 12 \pi R\right.$ and $\left.20 \pi R\right)$ tested approach zero around $z / R=10$, indicating a minimum computational pipe length of approximately $L_{z}=20 R$ (or, $6.4 \pi R$ ) is sufficient (and therefore, these three pipes lengths are proper), if the judgment is solely based on the two-point auto-correlation coefficient. However, in the following, I will show that this conclusion does not hold if I further consider the pre-multiplied energy spectra.

Figure 3.2 compares the profiles and isopleths of the pre-multiplied energy spectra for five different computational domain lengths at $R o_{\tau}=0.2$. The profiles in Fig. 3.2(a) are plotted along the axial direction of the pipe at wall-normal position $y^{+}=14.24$, while the isopleths in Fig. 3.2(b) are plotted with respect to both wavelength $\lambda_{z}^{+}$and wall coordinate $y^{+}$, which vividly show the near-wall effect on the scale of turbulence structures. In Fig. $3.2, k_{z}$ represents the axial wavenumber; $\phi_{z z}$, $\phi_{r r}$ and $\phi_{\theta \theta}$ represent the axial energy spectra of the three fluctuating velocity components; and the axial wavelength is defined as $\lambda_{z}=2 \pi / k_{z}$, non-dimensionalized as $\lambda_{z}^{+}=\lambda_{z} u_{\tau} / \nu$. From Fig. 3.2(a), it is clear that at $y^{+}=14.24$, the value of $k_{z} \phi_{z z}$ peaks at $\lambda_{z}^{+} \approx 700 \sim 2000$, while the values of $k_{z} \phi_{r r}$ and $k_{z} \phi_{\theta \theta}$ are comparatively small. In the case of the shortest pipe length $\left(L_{z}=2 \pi R\right)$, DNS fails to predict the peak value of the energy spectrum, and all turbulence kinetic energy (TKE) loses at the wavelengths larger than the domain size. In the case of the pipe lengths of $L_{z}=5 \pi R, 8 \pi R$ and $12 \pi R$, considerable amounts of energy (about $92.03 \%, 62.21 \%$ and $39.86 \%$ of the peak value of $k_{z} \phi_{z z}$, respectively) are not captured because the wavelengths of these 


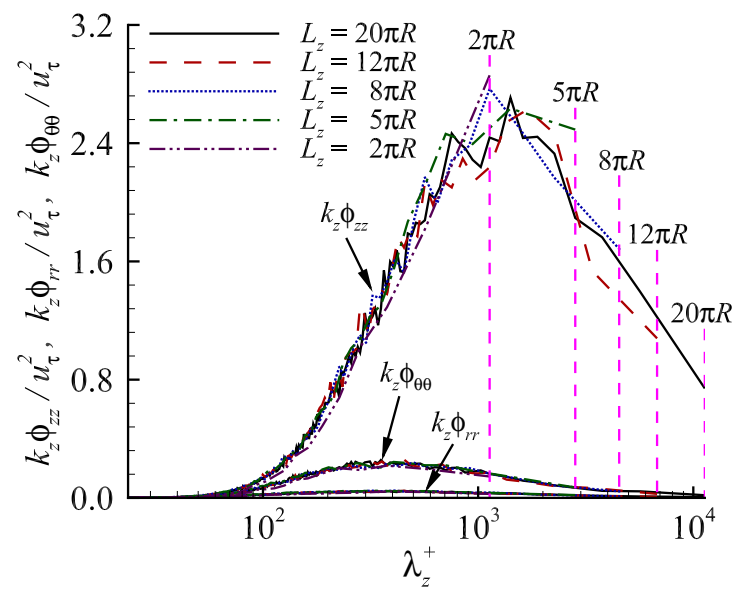

(a) Pre-multiplied energy spectra along the axial line of the pipe at wall-normal position $y^{+}=14.24$ from the pressure side $\left(\theta=90^{\circ}\right)$

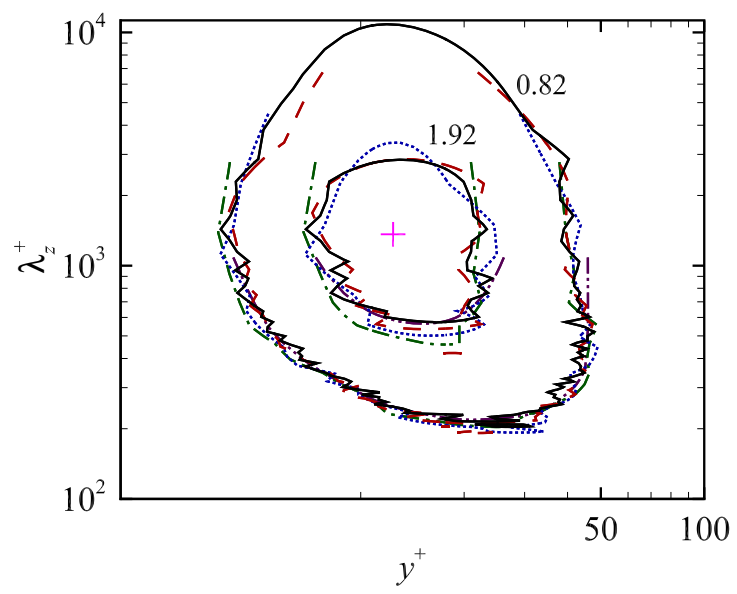

(b) Isopleths of pre-multiplied energy spectra $k_{z} \phi_{z z}$ in the central vertical plane $\left(\theta=90^{\circ}\right)$

Figure 3.2: Pre-multiplied energy spectra for different computational domain sizes $L_{z}$ at $R o_{\tau}=0.2$. The vertical dashed lines in panel (a) demarcate the computational domain sizes of $L_{z}=2 \pi R, 5 \pi R, 8 \pi R, 12 \pi R$, and $20 \pi R$, respectively. The isopleths in panel (b) are plotted with respect to wavelength $\lambda_{z}^{+}$and wall coordinate $y^{+}$. The cross symbol "+" in panel (b) indicates the location $\left(y^{+}=12.99, \lambda_{z}^{+}=1407.83\right)$ of the peak value of $k_{z} \phi_{z z}$. The value of the inner and outer isopleths is 1.92 and 0.82 (corresponding to $70 \%$ and $30 \%$ of the peak value of $k_{z} \phi_{z z}$, respectively). The pre-multiplied energy spectra have been non-dimensionalized using $u_{\tau}^{2}$.

amounts of missed TKE are larger than the corresponding pipe lengths. In contrast, for the case of the longest pipe length of $L_{z}=20 \pi R$, a relatively small amount of TKE ( $27.35 \%$ of the peak value) is held by the scales longer than the pipe length. This indicates that the result of the case with the longest pipe length $\left(L_{z}=20 \pi R\right)$ are considered proper as only less than $30 \%$ of TKE is missed in the numerical simulation. Figure 3.2(a) is plotted along the axial line of the pipe at the vertical location of $y^{+}=14.24$, which does not show any wall anisotropic effect. To refine my study of the pre-multiplied energy spectra, it is necessary to consider how it varies in the wall-normal direction. Figure 3.2(b) shows the isopleths of the pre-multiplied energy spectra of the axial velocity component $\left(k_{z} \phi_{z z}\right)$ on the pressure side of the rotating 
pipe as a function of both wavelength $\lambda_{z}^{+}$and wall coordinate $y^{+}$. Only the pressure side (for $0 \leq r \leq R$ or $0 \leq y^{+} \leq 180$ ) is considered because in a radially-rotating pipe flow, the flow is more turbulent on the pressure side (see the profile of $u_{z, r m s}^{\prime+}$ shown in Fig. 3.1(a)). The value of the pre-multiplied energy spectra peaks around $y^{+}=12.99$ and $\lambda_{z}^{+}=1407.83$. In contrast, for the non-rotating pipe flow tested, the spectral peak occurs at $y^{+}=14.24$ and $\lambda_{z}^{+}=1023.88$, which is similar to the result of Chin et al. [11] who demonstrated that the pre-multiplied energy spectra occurs at $y^{+} \approx 15$ and $\lambda_{z}^{+} \approx 1000$ in a non-rotating pipe flow of a similar Reynolds number. It is apparent that the characteristic streamwise length scale of the most energetic vortices increases (from $\lambda_{z}^{+}=1023.88$ to 1407.83 ) as the rotation number increases from $R o_{\tau}=0$ to 0.2 as a consequence of the Coriolis effects associated with the radial system rotation of the pipe. As shown in Fig. 3.2(b), the cases of $L_{z}=2 \pi R$ and $5 \pi R$ fail to capture the complete isopleth at a rather high TKE level of $k_{z} \phi_{z z} / u_{\tau}^{2}=1.92$ (or $70 \%$ of the peak value), and the cases of $L_{z}=8 \pi R$ and $12 \pi R$ start to fail at level of $k_{z} \phi_{z z} / u_{\tau}^{2}=0.82$ (or $30 \%$ of the peak value). In conclusion, in order to fully capture the scale of energetic eddy motions in different wall layers on the pressure side of the pipe, a very long pipe of $L_{z}=20 \pi R$ is needed, which allows for capturing the complete isopleth of the pre-multiplied energy spectrum up to $72.65 \%$ of its peak value.

\subsection{Instantaneous flow}

Figure 3.3 displays typical instantaneous axial velocity field $u_{z}^{+}$in a cross-stream plane at four different rotation numbers. The contour at each rotation number is presented at the same instant from the start of the simulation. As is clear from Fig. 3.3(a), typical of a non-rotating turbulent pipe flow, the flow structures are uniform in the azimuthal direction. Large-scale high-momentum flow structures dominate (indicted 


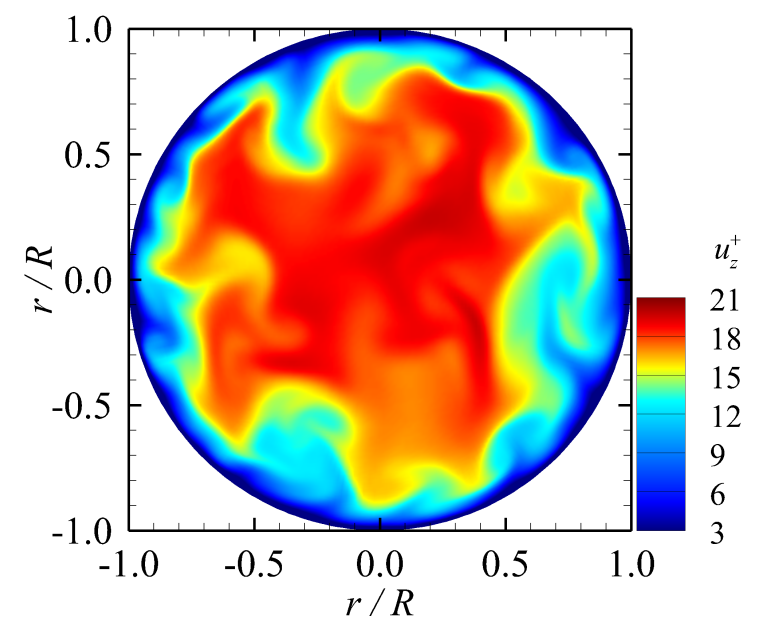

(a) $R o_{\tau}=0.0$

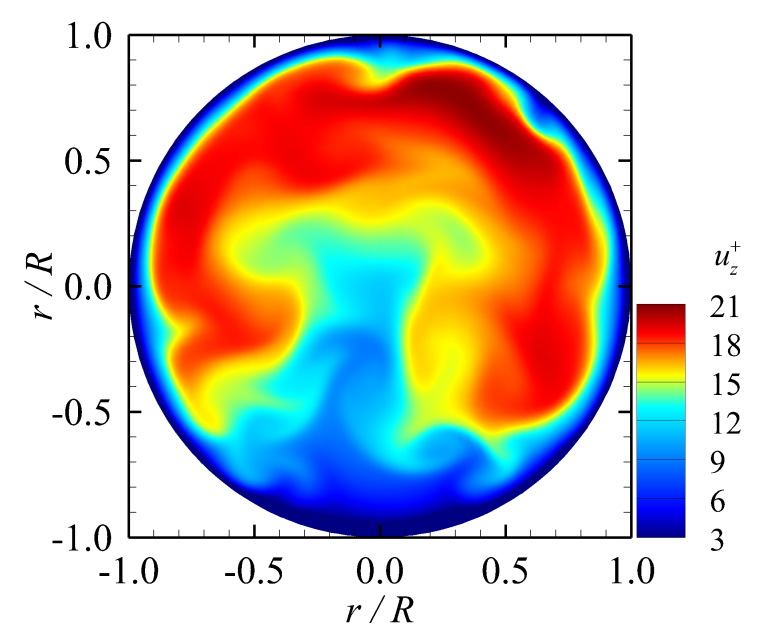

(c) $R o_{\tau}=0.8$

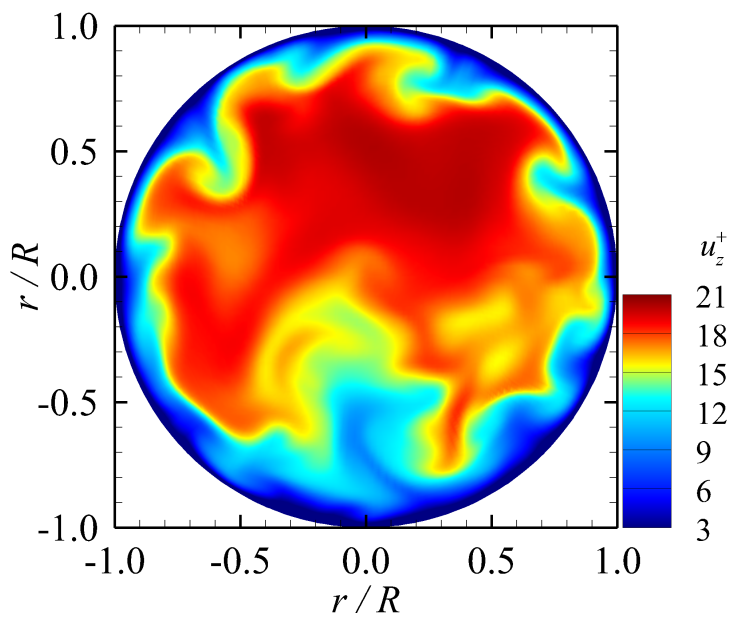

(b) $R o_{\tau}=0.2$

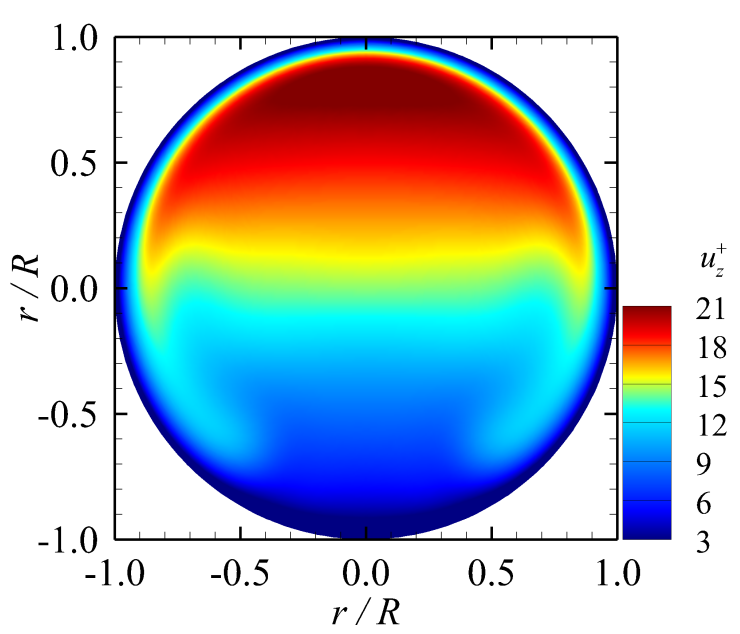

(d) $R o_{\tau}=1.0$

Figure 3.3: Contours of the instantaneous axial velocity $u_{z}^{+}$in a cross-stream plane at four different rotation numbers.

by red color) the central region of the pipe, while low-momentum flow structures (represented by blue color) are concentrated in the near-wall region of the curved pipe. It is interesting to observe that instantaneous flow structures exhibit "mushroom patterns", an observation that is consistent with the findings of Wu and Moin [10] who performed DNS study of a non-rotating pipe flow at the same Reynolds number. From Figs. 3.3(b)-(d), it is evident that the flow field becomes apparently asymmetric in the 
vertical direction once the radial system rotation is imposed. As the rotation number increases from $R o_{\tau}=0.2$ to 1.0, the pressure (or top) and suction (or bottom) sides of the pipe become increasingly dominated by high- and low-speed flows, respectively. Clearly, the role of the Coriolis force is to push the high-momentum fluids towards the pressure side and low-momentum fluids towards the suction side of the pipe. Given the circular boundary of the pipe, it is interesting to observe that there are two "legs" of the high-momentum fluid structures appearing on both sides of the pipe, a pattern that is the most apparent in Fig. 3.3(c). Finally, at $R o_{\tau}=1.0$ as shown in Fig. 3.3(d), the high- and low-momentum fluids occupy the pressure and suction sides of the pipe, respectively; and furthermore, the irregular mushroom structures vanish and the instantaneous flow field becomes apparently laminarized. The interesting instantaneous secondary flow patterns observed at four different rotation numbers in Fig. 3.3 can be more clearly demonstrated and better explained based on the mean velocity field in the following context.

\subsection{Mean flow}

Figure 3.4 compares the mean axial velocity fields $\left(\left\langle u_{z}\right\rangle^{+}\right)$of four different rotation numbers in a cross-stream plane of the pipe. Given the axial symmetry of the mean flow, only one half of the domain is plotted for each rotation number. As is evident in Fig. 3.4(a), at $R o_{\tau}=0.0$, the contour of the mean axial flow field exhibits a concentric pattern characteristic of a non-rotating Poiseuille pipe flow. As $R o_{\tau}$ increases to 0.2, mean secondary flows appear as a pair of counter-rotating vortices near the suction (bottom) side of the pipe in response to the radial system rotation imposed. Clearly, it is this pair of vortices that push the high-speed fluids in the pipe center $(r / R \rightarrow 0)$ towards the pressure side, and drive the low-momentum fluids along the periphery $(r / R \rightarrow 1)$ of the pipe towards the suction side. The large-scale vortex pair of the 


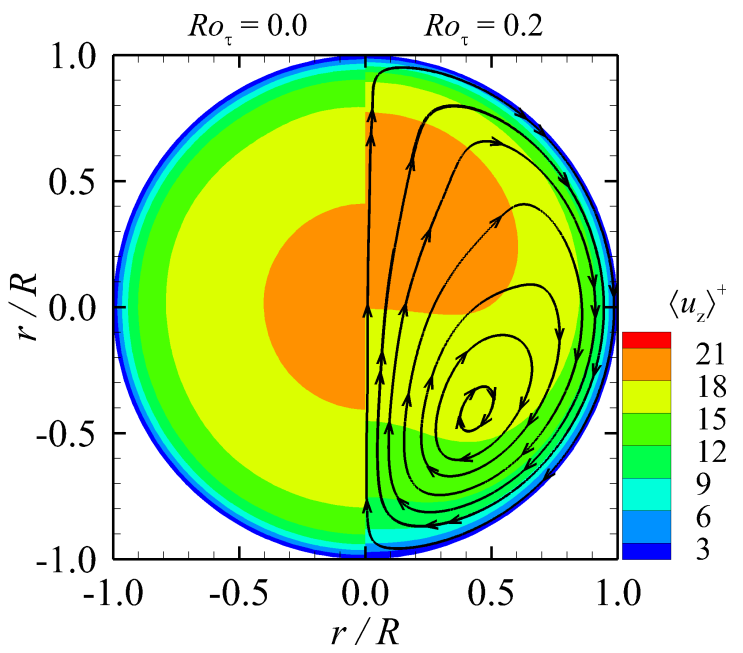

(a) $R o_{\tau}=0.0$ and 0.2

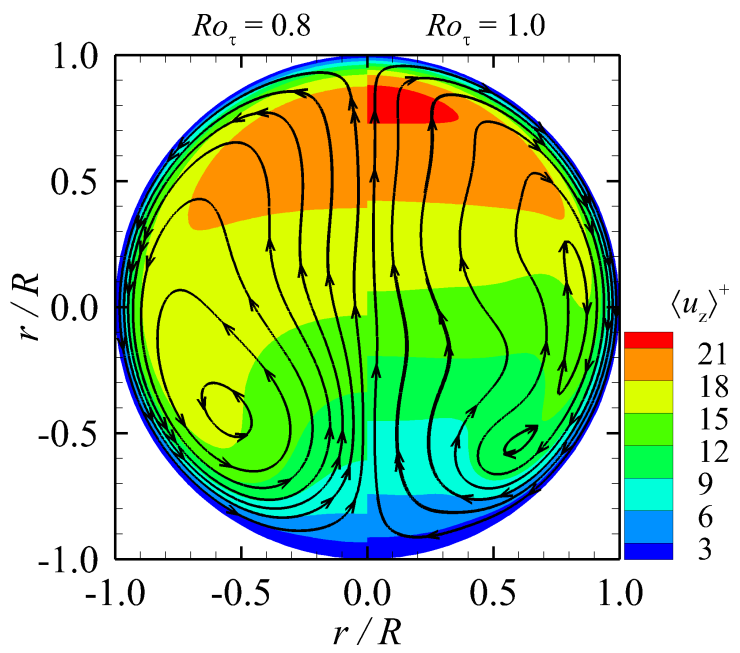

(b) $R o_{\tau}=0.8$ and 1.0

Figure 3.4: Contours of the mean axial velocity $\left\langle u_{z}\right\rangle^{+}$superimposed with mean streamlines in the cross-stream plane at different rotation numbers.

mean flow observed here well explains the instantaneous secondary flow structures shown in Fig. 3.3(b). In addition, a small quasi TP region (defined by the invariant mean axial velocity along the rotating direction, featuring $\partial\left\langle u_{z}\right\rangle / \partial x \approx 0$ ) forms in the pipe center. As $R o_{\tau}$ reaches 0.8, the quasi TP region expands further along the rotating axis. The mean secondary flow pattern is similar between $R_{\tau}=0.2$ and 0.8 , featuring only one pair of counter-rotating vortices. Because the mean axial Coriolis force $\left\langle C_{z}\right\rangle$ points to the negative $z$-direction, it suppresses the axial momentum of the turbulent flow. As is clear in Fig. 3.4(b), at $R o_{\tau}=1.0$, the flow becomes fully laminarized, and two pairs of counter-rotating roll cells appear in the mean secondary flow.

Figure 3.5 compares the bulk mean velocity $U_{b}^{+}$, volume-averaged TKE $k_{m}^{+}$and skin friction coefficient $C_{f}$ with respect to 11 rotation numbers. From Fig. 3.5(a), it is seen that as the rotation number increases from $R o_{\tau}=0.0$ to 1.0 , the profile of $U_{b}^{+}$varies non-monotonically. At $R o_{\tau}=0.3$, the value of $U_{b}^{+}$reaches its maximum, which is $2.87 \%$ higher than that of the non-rotating case $\left(R o_{\tau}=0.0\right)$. However, at the 


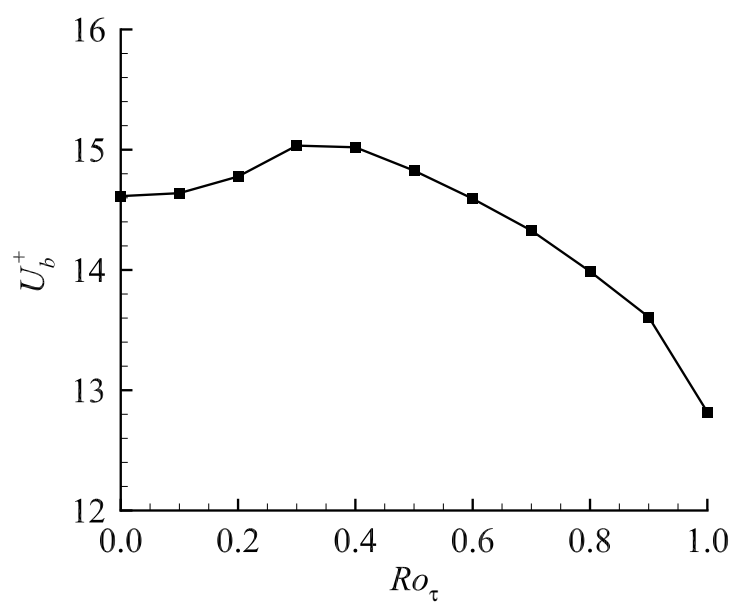

(a) Bulk mean velocity $U_{b}^{+}$

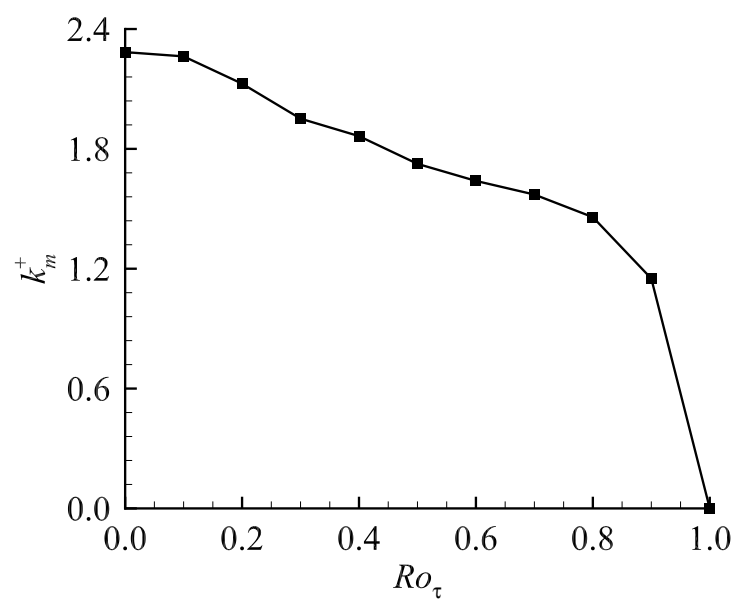

(b) Volume-averaged TKE $k_{m}^{+}$

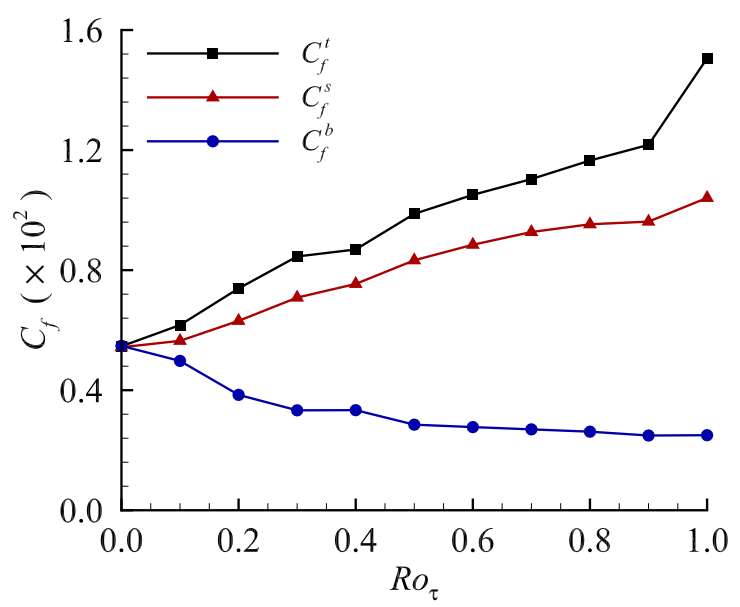

(c) Skin friction coefficient $C_{f}$

Figure 3.5: Comparison of bulk mean velocity $U_{b}^{+}$, volume-averaged TKE $k_{m}^{+}$, and skin friction coefficient $C_{f}$ as a function of the rotation number. The TKE value has been non-dimensionalized using $u_{\tau}^{2}$.

highest rotation number $R o_{\tau}=1.0$, the magnitude of $U_{b}^{+}$is $12.28 \%$ lower than that of the non-rotating case. It is interesting that the trend of $U_{b}^{+}$in the turbulent circular pipe flow is qualitatively different from those of Grundestam et al. [3] for turbulent plane-channel flow and Fang et al. [8] for turbulent square duct flow in the sense that the value of $U_{b}^{+}$decreases monotonically with an increasing rotation number in the 
latter two canonical flows (owing to the different geometry setups of the computational domains and boundary conditions). In contrast to the bulk mean velocity profile, from Fig. 3.5(b), it is evident that the value of $k_{m}^{+}$decreases monotonically with an increasing rotation number, implying that the Coriolis force resulted from radial system rotation always acts to damp out the general turbulence level of the pipe flow. Moreover, as the rotation number increases beyond $R o_{\tau}=0.9$, the decay of $k_{m}^{+}$is so dramatic, and eventually, the volume-averaged TKE value becomes zero at $R o_{\tau}=1.0$, clearly indicating that the flow field has become completely laminarized. The reason for this dramatically decreasing trend is that the laminarization process is accelerated due to the increasing Coriolis effects at a high rotation number. Figure 3.5(c) shows the variation of the skin friction coefficient as a function of the rotation number. In the figure, $C_{f}^{t}, C_{f}^{s}$ and $C_{f}^{b}$ represent skin friction coefficient at the top (or pressure side, at $\theta=90^{\circ}$ ), lateral sides (at $\theta=0^{\circ}$ or $180^{\circ}$ ), and bottom (or suction side, at $\theta=270^{\circ}$ ) of the pipe. From Fig. 3.5(c), as $R o_{\tau}$ increases from 0.0 to 1.0, the magnitudes of $C_{f}^{t}$ and $C_{f}^{s}$ increase monotonically, however, that of $C_{f}^{b}$ decreases monotonically. The sharp contrast between the trends of $C_{f}^{t}$ and $C_{f}^{b}$ can be well explained by the secondary flow patterns shown in Figs. 3.3 and 3.4. As the rotation number increases, the Coriolis force effects (and the strength of the associated secondary flows) become increasingly stronger, which push high- and low-axial-moment fluids to the pressure and suction sides, respectively. This further necessarily leads to increased and decreased values in $C_{f}^{t}$ and $C_{f}^{b}$, respectively.

Figure 3.6 compares the profiles of the mean axial velocity $\left\langle u_{z}\right\rangle^{+}$for six different rotation numbers in the central vertical plane $\left(\theta=90^{\circ}\right)$ and in the central horizontal plane $\left(\theta=0^{\circ}\right)$, respectively. As is clear in Fig. 3.6(a), the profile of $\left\langle u_{z}\right\rangle^{+}$is perfectly symmetrical for the non-rotating case $\left(R o_{\tau}=0.0\right)$. However, as $R o_{\tau}$ increases, the profile becomes increasingly asymmetrical, and the peak value of $\left\langle u_{z}\right\rangle^{+}$is shifted from the pipe center to the pressure side of the pipe due to secondary flows in the 


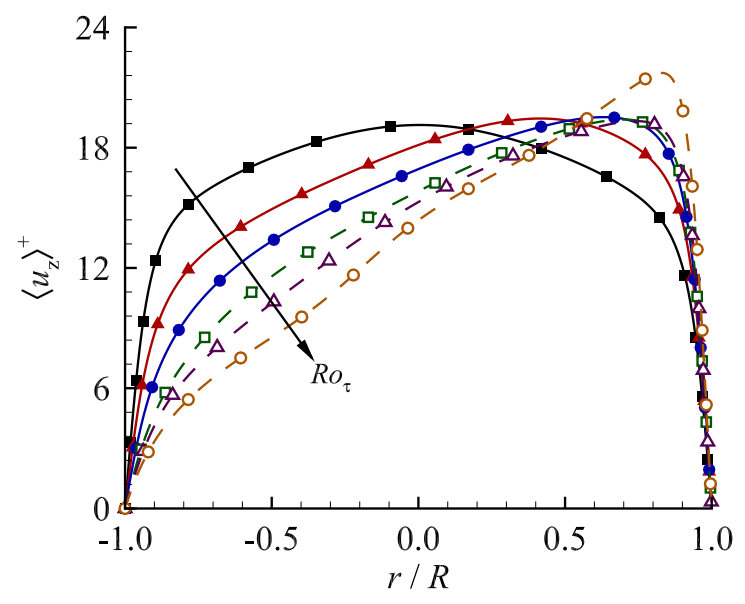

(a) Central vertical plane at $\theta=90^{\circ}$

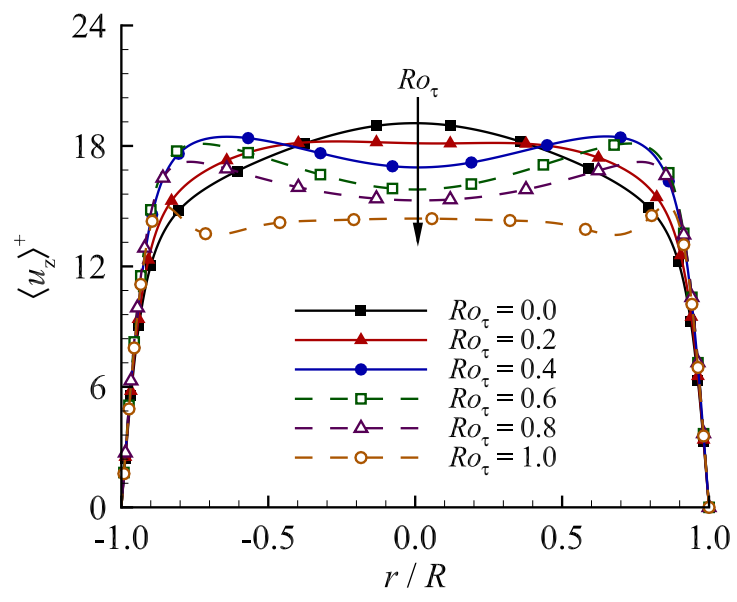

(b) Central horizontal plane at $\theta=0^{\circ}$

Figure 3.6: Profiles of mean axial velocity $\left\langle u_{z}\right\rangle^{+}$at six different rotation numbers. Arrow points to the direction of monotonic variation of the rotation number.

cross-stream direction (shown in Fig. 3.4). Meanwhile, the magnitude of $\left\langle u_{z}\right\rangle^{+}$decreases monotonically with an increasing $R o_{\tau}$ near the suction side of the pipe. From Fig. 3.6(b), it is clear that as rotation number increases from $R o_{\tau}=0.0$ to 1.0 , the magnitude of the mean streamwise velocity $\left\langle u_{z}\right\rangle^{+}$decreases monotonically in the pipe center. Furthermore, it is observed that as the rotation number reaches 0.2 , a quasi TP region previously observed qualitatively in Fig. 3.4 also appears in Fig. 3.6(b), indicated by the fact that the profile of $\left\langle u_{z}\right\rangle^{+}$tends to be "flat" (i.e., the profile is insensitive to the $x$-direction, or the rotating direction) in the pipe center. However, owing to presence of the curved surface of the pipe wall, an ideal TP region (strictly obeying $\left.\partial\left\langle u_{z}\right\rangle^{+} / \partial x \equiv 0\right)$ is not observed, which is qualitatively different from the spanwise-rotating 2-D plane channel flow case of Kristoffersen and Andersson [2] and Johnston et al. [29]. 


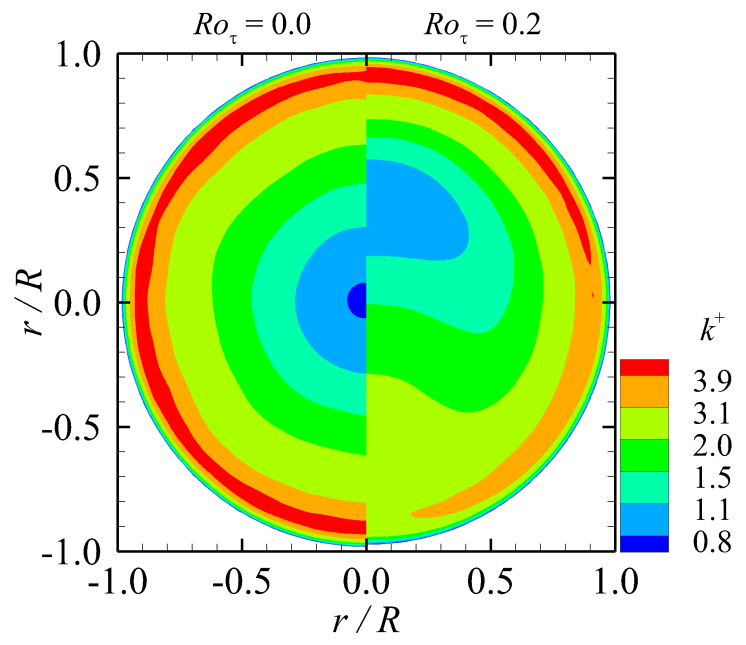

(a) $R o_{\tau}=0.0$ and 0.2

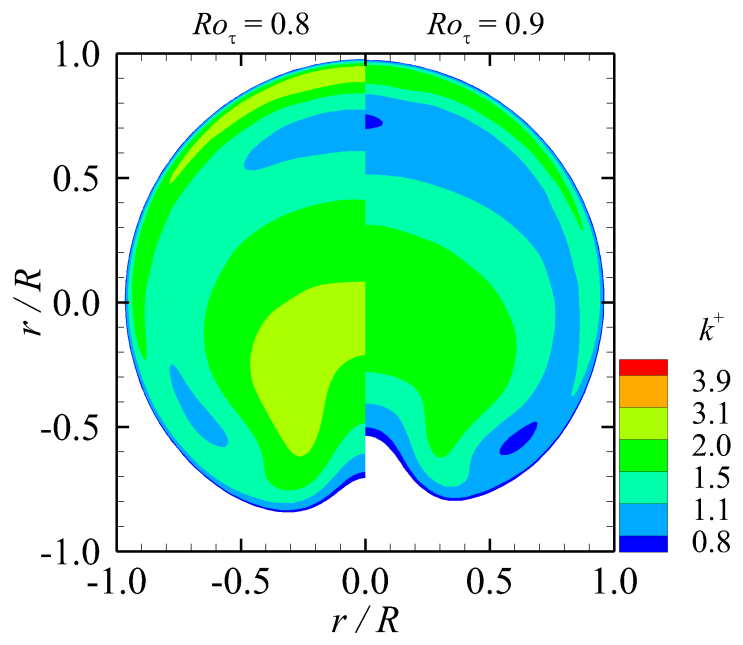

(b) $R o_{\tau}=0.8$ and 0.9

Figure 3.7: Contours of TKE $k^{+}$in a cross-stream plane at four different rotation numbers. The contour legends are kept identical in panels (a) and (b) in order to facilitate a direct comparison of TKE levels at four rotation numbers. Furthermore, contours corresponding to low TKE levels (for $k^{+} \leq 0.7$ ) are clipped to highlight turbulent regions. The TKE value has been non-dimensionalized using $u_{\tau}^{2}$.

\subsection{Turbulence statistics}

Figure 3.7 compares the contours of TKE $k=\left\langle u_{i}^{\prime} u_{i}^{\prime}\right\rangle / 2$ (non-dimensionalized by $u_{\tau}^{2}$, i.e. $\left.k^{+}=k / u_{\tau}^{2}\right)$ in a cross-stream plane at four of the eleven rotation numbers tested. As is clear from Fig. 3.7(a), for a non-rotating flow $\left(R o_{\tau}=0.0\right)$, the contours of the TKE field exhibit a concentric pattern in the cross-stream plane as a direct result of perfect axial-symmetry of the flow and boundary conditions. The maximal and minimal TKE values occur in the buffer layer (for $y^{+}=12-22$ ) and at the pipe center, respectively. Clearly, the axial-symmetry is destroyed as soon as the system rotation is imposed. At $R_{\tau}=0.2$, the TKE level reduces near the lateral sides and bottom wall. In Fig. 3.7(b), as rotation number further increases to $R o_{\tau}=0.8$ and 0.9 , the reduction in the TKE value becomes very significant as the Coriolis force strengthens. In order to make a fair comparison of the TKE levels at four rotation numbers, the 


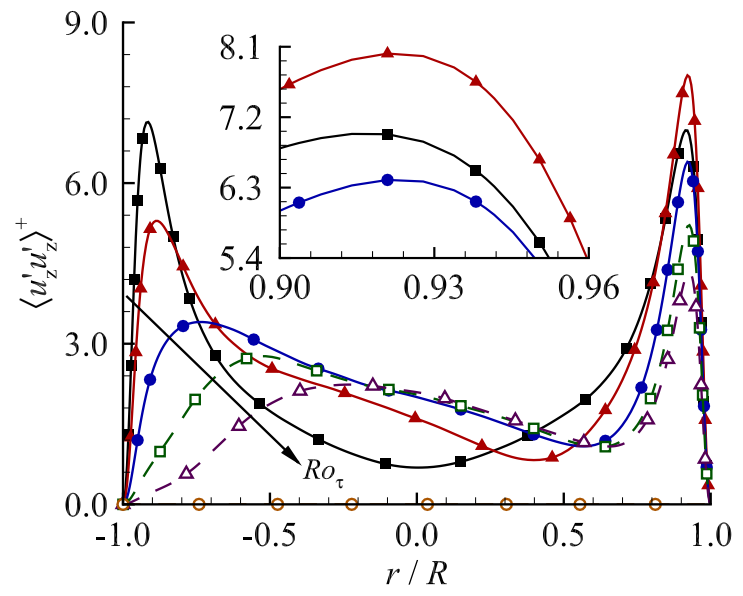

(a) $\left\langle u_{z}^{\prime} u_{z}^{\prime}\right\rangle^{+}$

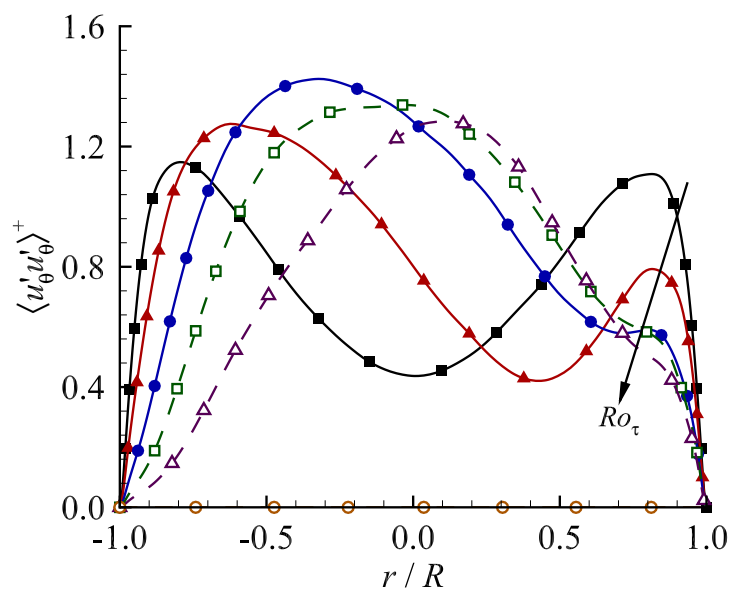

(c) $\left\langle u_{\theta}^{\prime} u_{\theta}^{\prime}\right\rangle^{+}$

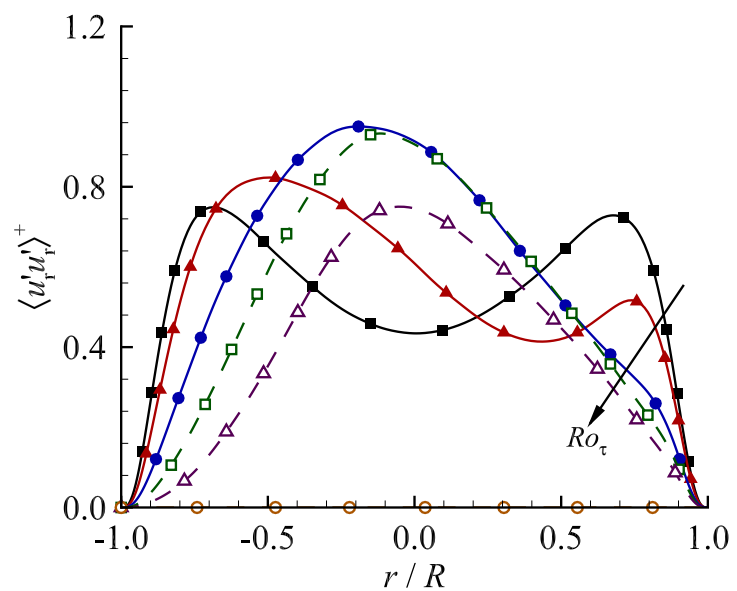

(b) $\left\langle u_{r}^{\prime} u_{r}^{\prime}\right\rangle^{+}$

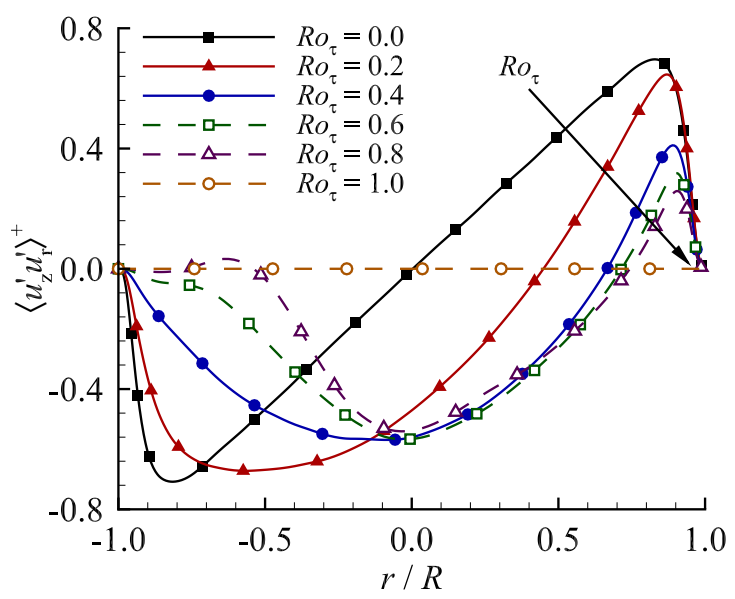

(d) $\left\langle u_{z}^{\prime} u_{r}^{\prime}\right\rangle^{+}$

Figure 3.8: Profiles of Reynolds stresses in the central vertical plane $\left(\theta=90^{\circ}\right)$ of the pipe at different rotation numbers. Arrow points to the direction of an increasing rotation number.

contour legends are kept identical at four rotation numbers in Fig. 3.7. Furthermore, contours levels corresponding to low TKE levels (for $k^{+} \leq 0.7$ ) have been clipped off in order to highlight the turbulent regions and the trend of laminarization. The Coriolis effects on the distribution of the TKE and laminarization of the flow observed here are qualitatively similar to those observed in turbulent plane-channel flow (Grundestam et al. [3]) and square-duct flow (Fang et al. [8]) under spanwise system rotations. 
Among the six independent Reynolds stress components, $\left\langle u_{\theta}^{\prime} u_{r}^{\prime}\right\rangle \equiv 0$ and $\left\langle u_{\theta}^{\prime} u_{z}^{\prime}\right\rangle \equiv$ 0 due to the axial-symmetry of the pipe flow. Figure 3.8 compares the profiles of the other four non-trivial stress components, $\left\langle u_{z}^{\prime} u_{z}^{\prime}\right\rangle,\left\langle u_{r}^{\prime} u_{r}^{\prime}\right\rangle,\left\langle u_{\theta}^{\prime} u_{\theta}^{\prime}\right\rangle$ and $\left\langle u_{z}^{\prime} u_{r}^{\prime}\right\rangle$, in the central vertical plane $\left(\theta=90^{\circ}\right)$ of the pipe for six different rotation numbers. It is clear that for a non-rotating flow, the profiles of these four non-trivial Reynolds stresses exhibit central-symmetry, which however, breaks as soon as the radial system rotation is imposed. From Figs. 3.8(b)-(d), it is seen that in the near-wall region of the pressure side $(0 \leq r / R \leq 1)$ of the pipe, the peak value of $\left\langle u_{r}^{\prime} u_{r}^{\prime}\right\rangle,\left\langle u_{\theta}^{\prime} u_{\theta}^{\prime}\right\rangle$ and $\left\langle u_{z}^{\prime} u_{r}^{\prime}\right\rangle$ decreases monotonically as the rotation number increases. However, it is interesting to observe in Fig. 3.8(a) that the peak value of the streamwise Reynolds normal stress component $\left\langle u_{z}^{\prime} u_{z}^{\prime}\right\rangle$ occurs at $R o_{\tau}=0.2$ at $r / R=0.921$ (or $y^{+}=14.24$ ). Furthermore, in the near-wall region of the suction side $(-1 \leq r / R \leq 0)$, the peak value of the $\left\langle u_{z}^{\prime} u_{z}^{\prime}\right\rangle$ decreases monotonically with an increasing rotation number. Nevertheless, in Figs. 3.8(b)-(d), the peak value of $\left\langle u_{r}^{\prime} u_{r}^{\prime}\right\rangle,\left\langle u_{\theta}^{\prime} u_{\theta}^{\prime}\right\rangle$ and $\left\langle u_{z}^{\prime} u_{r}^{\prime}\right\rangle$ varies non-monotonically and its position consistently shifts towards the pipe center as the rotation number increases. As shown in Figs. 3.8(b) and (c), the profiles of $\left\langle u_{r}^{\prime} u_{r}^{\prime}\right\rangle$ and $\left\langle u_{\theta}^{\prime} u_{\theta}^{\prime}\right\rangle$ exhibit a characteristic dual-peak pattern to reflect wall-anisotropic effects for non-rotating $\left(R o_{\tau}=0\right)$ and rotating pipe flows at relatively low rotation numbers $\left(R o_{\tau} \leq 0.2\right)$. However, as the rotation number increases beyond 0.2 , the profiles of $\left\langle u_{r}^{\prime} u_{r}^{\prime}\right\rangle$ and $\left\langle u_{\theta}^{\prime} u_{\theta}^{\prime}\right\rangle$ transform from a dual-peak pattern to a single-peak pattern, reflecting the effect of the Coriolis force. As is evident from Fig. 3.8(d), as soon as the radial system rotation is imposed on the pipe flow, the well-recognized linear distribution of the Reynolds shear stress $\left\langle u_{z}^{\prime} u_{r}^{\prime}\right\rangle$ characteristic of a non-rotating pipe flow starts to lose effect. As the rotation number increases, the strength of $\left\langle u_{z}^{\prime} u_{r}^{\prime}\right\rangle$ decreases on both sides of the pipe. Eventually, as $R o_{\tau}$ approaches 1.0, the value of the Reynolds shear stress becomes trivial, indicating that the flow becomes fully laminarized by the Coriolis force. 


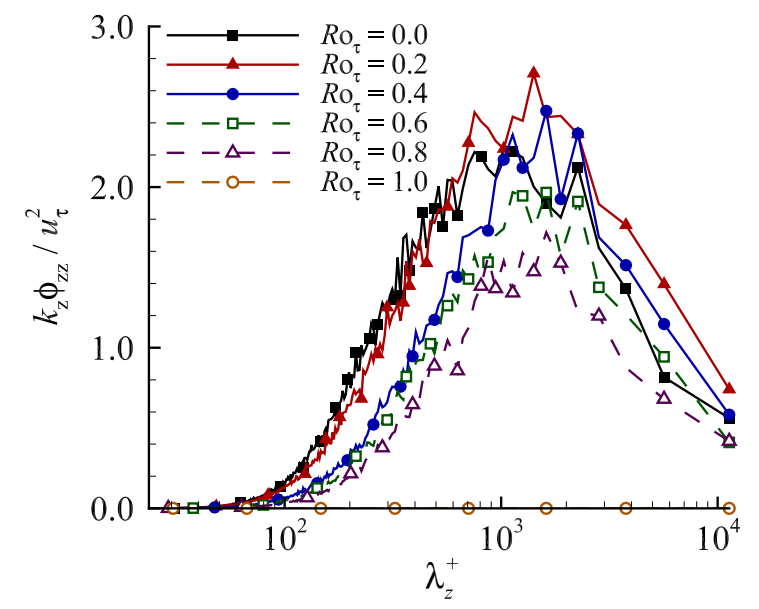

(a) Axial component

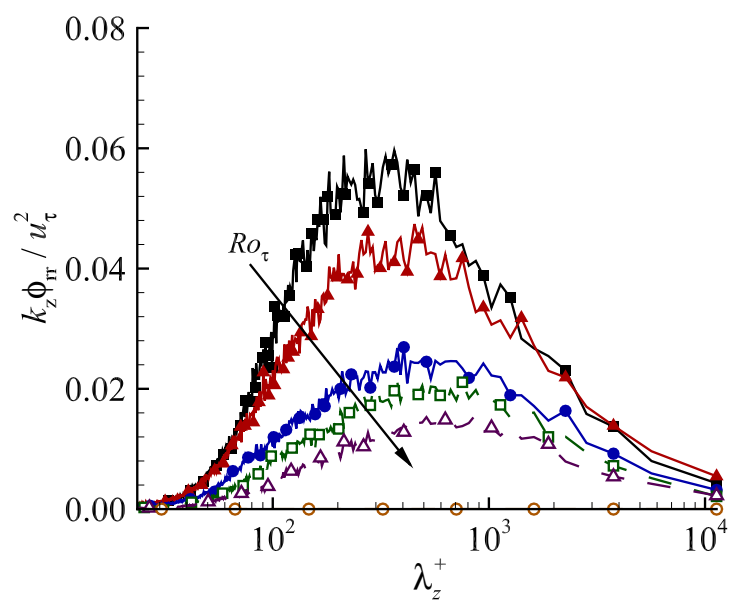

(b) Radial component

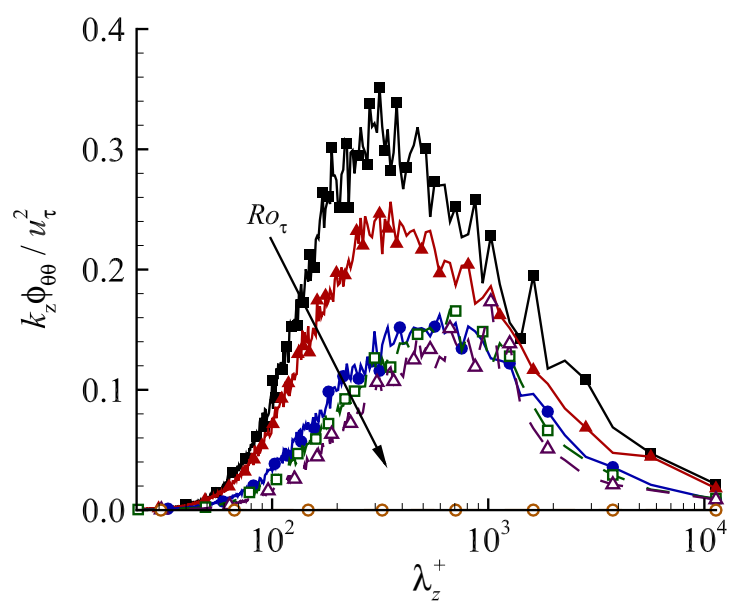

(c) Azimuthal component

Figure 3.9: Effect of radial system rotation on the pre-multiplied velocity spectra calculated along the axial line of the pipe at wall-normal position $y^{+}=14.24$ on the pressure side $\left(\theta=90^{\circ}\right)$ of the pipe. Arrow points to the direction of an increasing rotation number. The pre-multiplied energy spectra $k_{z} \phi_{i i}$ (no summation convention implied here) have been non-dimensionalized using $u_{\tau}^{2}$.

Figure 3.9 compares pre-multiplied spectra of axial, radial and azimuthal velocity fluctuations $\left(k_{z} \phi_{z z}, k_{z} \phi_{r r}\right.$ and $k_{z} \phi_{\theta \theta}$, respectively) calculated along the axial line at wall-normal position $y^{+}=14.24$ on the pressure side $\left(\theta=90^{\circ}\right)$ of the pipe for six different rotation numbers. From Fig. 3.9, it is evident that the magnitude of $k_{z} \phi_{z z}$ is 
much higher than those of $k_{z} \phi_{r r}$ and $k_{z} \phi_{\theta \theta}$. As is clear in Fig. 3.9(a), the magnitude of $k_{z} \phi_{z z}$ varies non-monotonically with respect to $R o_{\tau}$ at large wavelengths. A perusal of the figure indicates that the peak value of $k_{z} \phi_{z z}$ reaches the largest at $R o_{\tau}=0.2$. By contrast, at small wavelengths, the magnitude of $k_{z} \phi_{z z}$ decreases monotonically with an increasing $R o_{\tau}$, indicating that the Coriolis force tends to suppress turbulent motions at small scales in the axial direction. As is evident from Fig. 3.9(b) and (c), the magnitudes of $k_{z} \phi_{r r}$ and $k_{z} \phi_{\theta \theta}$ both decrease monotonically with an increasing rotation number. This indicates that the general effect of radial system rotation is to suppress turbulent motions (at all scales) in the radial and azimuthal directions. Furthermore, it is observed that the characteristic axial length scales (as indicated by the modes of $k_{z} \phi_{r r}$ and $\left.k_{z} \phi_{\theta \theta}\right)$ corresponding to the most energetic eddies increase monotonically from $\lambda_{z}^{+}=200 \sim 600$ to $400 \sim 1000$ as the rotation number increases from $R o_{\tau}=0.0$ to 0.8. However, as shown in Figs. 3.9(a)-(c), once the rotation number continues to increase to $R o_{\tau}=1.0$, the pre-multiplied spectra of all three fluctuating velocity components become zero identically, clearly indicating a full laminarization state of the flow at the highest rotation number tested. By comparing Figs. 3.9(a)(c), it is clear that the TKE level associated with axial velocity fluctuations is the highest among the three velocity components because the magnitude of $k_{z} \phi_{z z}$ is one or two orders larger than those of $k_{z} \phi_{r r}$ and $k_{z} \phi_{\theta \theta}$. Furthermore, the energy loss at the cut-off wavelength (i.e., at $\left(\lambda_{z}^{+}\right)_{\max }=11309.73$, corresponding to the pipe length $L_{z}=20 \pi R$ ), is also the highest with axial velocity fluctuations. Specifically, the percentage of TKE loss (with respect to the peak value of the pre-multiplied spectrum) at the cutoff wavelength is $25.1 \%, 27.4 \%, 23.6 \%, 20.9 \%$ and $24.5 \%$ at $R o_{\tau}=0.0,0.2,0.4,0.6$ and 0.8 , respectively.

In order to refine my study of the effect of rotation on the Reynolds stresses, the transport equation of Reynolds stresses $\left\langle u_{i}^{\prime} u_{j}^{\prime}\right\rangle$ can be further studied, which is 
expressed as

$$
H_{i j}-P_{i j}-\Pi_{i j}+\varepsilon_{i j}-D_{i j}-C_{i j}=0
$$

Here, $H_{i j}, P_{i j}, \Pi_{i j}, \varepsilon_{i j}, D_{i j}$ and $C_{i j}$ represent the convection, production, pressurestrain, viscous dissipation, diffusion (consisting of turbulent, pressure and viscous diffusion effects) and Coriolis production terms, respectively. The definitions of these terms are complex, and are given in the appendix B.

Figures 3.10, 3.11 and 3.12 compare the budget terms of $\left\langle u_{z}^{\prime} u_{z}^{\prime}\right\rangle,\left\langle u_{r}^{\prime} u_{r}^{\prime}\right\rangle$ and $\left\langle u_{z}^{\prime} u_{r}^{\prime}\right\rangle$, respectively, in the central vertical plane $\left(\theta=90^{\circ}\right)$ of the pipe for the non-rotating and rotating cases (with $R o_{\tau}=0.0,0.2$ and 0.8). The budget terms of $\left\langle u_{\theta}^{\prime} u_{\theta}^{\prime}\right\rangle$ are not presented simply because the Coriolis effect is trivial (i.e., $C_{\theta \theta}=4 \Omega\left\langle u_{\theta}^{\prime} u_{z}^{\prime}\right\rangle \cos \theta=0$ ) in this central vertical plane. Also, the budget terms of $\left\langle u_{\theta}^{\prime} u_{r}^{\prime}\right\rangle$ and $\left\langle u_{\theta}^{\prime} u_{z}^{\prime}\right\rangle$ are not presented because these two Reynolds shear stresses are zero identically due to the axial symmetry condition of the pipe flow. For the three Reynolds stress components investigated, the Coriolis production term $C_{i j}$ appears in their transport equations as a result of radial system rotation, and according to the appendix B, they are

$$
\begin{aligned}
& C_{z z}=-4 \Omega\left(\left\langle u_{r}^{\prime} u_{z}^{\prime}\right\rangle \sin \theta+\left\langle u_{\theta}^{\prime} u_{z}^{\prime}\right\rangle \cos \theta\right) \\
& C_{r r}=4 \Omega\left\langle u_{r}^{\prime} u_{z}^{\prime}\right\rangle \sin \theta, \\
& C_{z r}=2 \Omega\left(\left\langle u_{z}^{\prime} u_{z}^{\prime}\right\rangle \sin \theta-\left\langle u_{r}^{\prime} u_{r}^{\prime}\right\rangle \sin \theta-\left\langle u_{r}^{\prime} u_{\theta}^{\prime}\right\rangle \cos \theta\right)
\end{aligned}
$$

In the central vertical plane $\left(\theta=90^{\circ}\right)$, the above equations are further simplified to

$$
\begin{aligned}
& C_{z z}=-4 \Omega\left\langle u_{r}^{\prime} u_{z}^{\prime}\right\rangle, \\
& C_{r r}=4 \Omega\left\langle u_{r}^{\prime} u_{z}^{\prime}\right\rangle, \\
& C_{z r}=2 \Omega\left(\left\langle u_{z}^{\prime} u_{z}^{\prime}\right\rangle-\left\langle u_{r}^{\prime} u_{r}^{\prime}\right\rangle\right) .
\end{aligned}
$$


Clearly, the joint function of $C_{z z}$ and $C_{r r}$ is to transfer turbulence energy between the two Reynolds normal components $\left\langle u_{z}^{\prime} u_{z}^{\prime}\right\rangle$ and $\left\langle u_{r}^{\prime} u_{r}^{\prime}\right\rangle$, simply because $C_{z z} \equiv-C_{r r}$. On the other hand, the Coriolis production term $C_{z r}$ behaves as an additional source term for the Reynolds shear stress component $\left\langle u_{z}^{\prime} u_{r}^{\prime}\right\rangle$. If there is a net energy balance between the $\left\langle u_{z}^{\prime} u_{z}^{\prime}\right\rangle$ and $\left\langle u_{r}^{\prime} u_{r}^{\prime}\right\rangle$, the Coriolis force (as indicated by the factor $2 \Omega$ in Eq. (3.7)) shifts this amount of energy balance $\left(\left\langle u_{z}^{\prime} u_{z}^{\prime}\right\rangle-\left\langle u_{r}^{\prime} u_{r}^{\prime}\right\rangle\right)$ to the Reynolds shear stress component $\left\langle u_{z}^{\prime} u_{r}^{\prime}\right\rangle$.

As shown in Figs. 3.10(a), (b) and (c), a common feature between the non-rotating and rotating pipe flows is that the budget balance is dominated by $-P_{z z}, \varepsilon_{z z}$ and $-D_{z z}$ in the near-wall region. However, at $R o_{\tau}=0.2$, the peak values of these three terms are much smaller on the suction side than on the pressure side. This indicates that owing to the system rotation imposed, the flow becomes more turbulent on the pressure side than on the suction side. As $R o_{\tau}$ reaches 0.8 , the magnitudes of these three terms on both sides reduce, demonstrating that the flow field is less turbulent and starts to become laminarized at a high rotation number. Similarly, by comparing Fig. 3.11(b) with 3.11(c) and Fig. 3.12(b) with 3.12(c), it is apparent that the magnitudes of the budget terms are smaller at $R o_{\tau}=0.8$ than at $R o_{\tau}=0.2$, further revealing a laminarization trend as the rotation number becomes high.

As is clear in Fig. 3.10(b), although the value of Coriolis production term $-C_{z z}$ is small, its sign changes at $r / R=0.45$. As such, it facilitates the Reynolds normal stress component $\left\langle u_{z}^{\prime} u_{z}^{\prime}\right\rangle$ to receive energy from another Reynolds normal stress component $\left\langle u_{r}^{\prime} u_{r}^{\prime}\right\rangle$ within the region $-1.0<r / R<0.45$, and to transfer energy back to the $\left\langle u_{r}^{\prime} u_{r}^{\prime}\right\rangle$ component within the region $0.45<r / R<1.0$ where $-C_{z z}$ behaves in a similar mechanism as the pressure-strain term $-\Pi_{z z}$. This property of $C_{z z}$ can be well explained through its definition $\left(C_{z z}=-4 \Omega\left\langle u_{z}^{\prime} u_{r}^{\prime}\right\rangle\right)$ and the profile of $\left\langle u_{z}^{\prime} u_{r}^{\prime}\right\rangle$ shown previously in Fig. 3.8(d). Clearly, at $R o_{\tau}=0.2$, the sign of $\left\langle u_{z}^{\prime} u_{r}^{\prime}\right\rangle$ changes at $r / R=0.45$, directly resulting in a change in the sign of $C_{z z}$ at the same position. By 


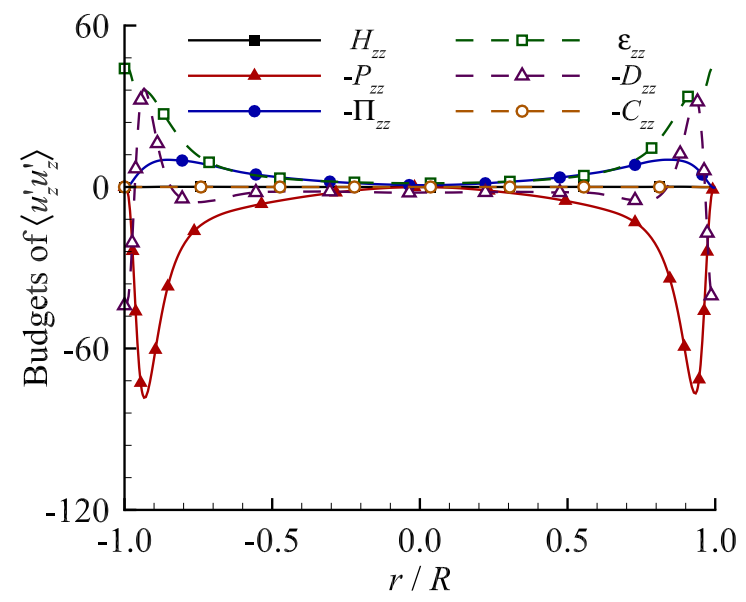

(a) $R o_{\tau}=0.0$

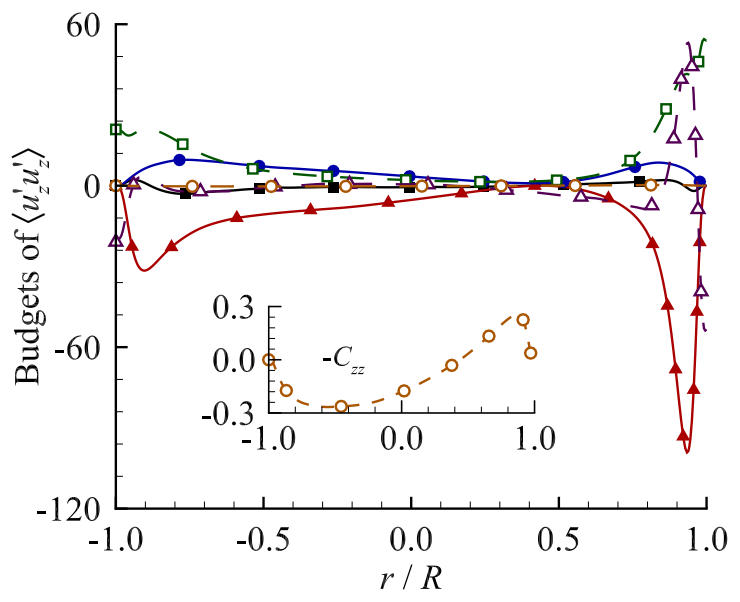

(b) $R o_{\tau}=0.2$

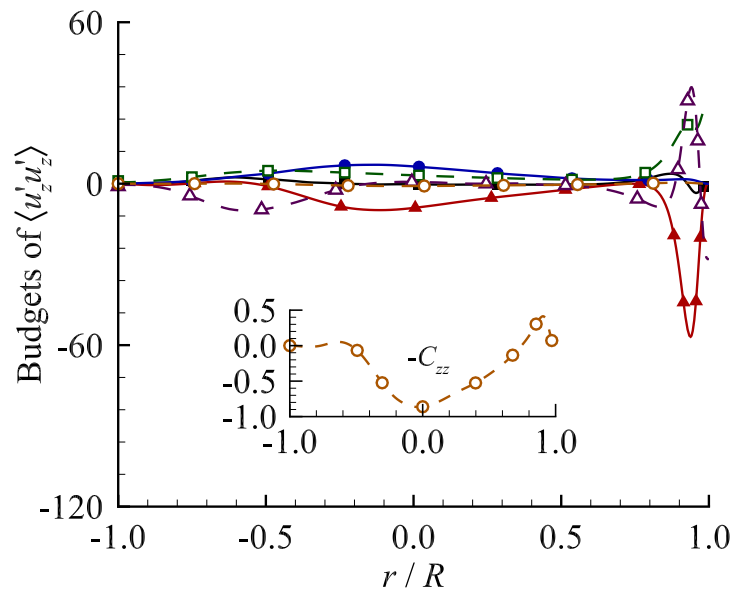

(c) $R o_{\tau}=0.8$

Figure 3.10: Profiles of the budget terms of $\left\langle u_{z}^{\prime} u_{z}^{\prime}\right\rangle$ in the central vertical plane $\left(\theta=90^{\circ}\right)$ of the pipe for different rotation numbers. All budget terms have been non-dimensionalized using $u_{\tau}^{3} / R$.

comparing Fig. 3.11(b) with 3.10(b) and Fig. 3.11(c) with 3.10(c), it is evident that the mechanism and trend of the Coriolis production term $-C_{r r}$ are opposite to those of $-C_{z z}$, simply because $C_{z z}=-C_{r r}$ holds in the vertical central plane $\left(\theta=90^{\circ}\right)$ by definition. Furthermore, as shown in Figs. 3.11(b) and (c), the budget balance of $\left\langle u_{r}^{\prime} u_{r}^{\prime}\right\rangle$ is dominated by the pressure-strain term $-\Pi_{r r}$ and dissipation term $\varepsilon_{r r}$. By 


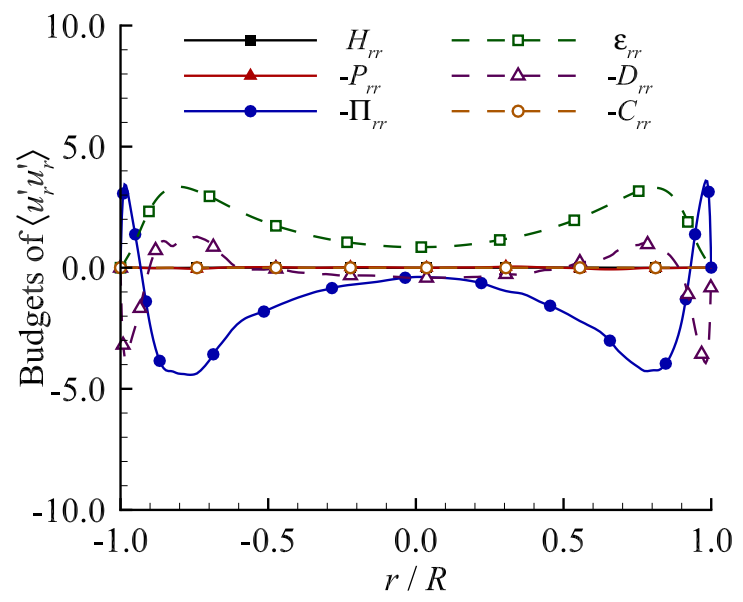

(a) $R o_{\tau}=0.0$

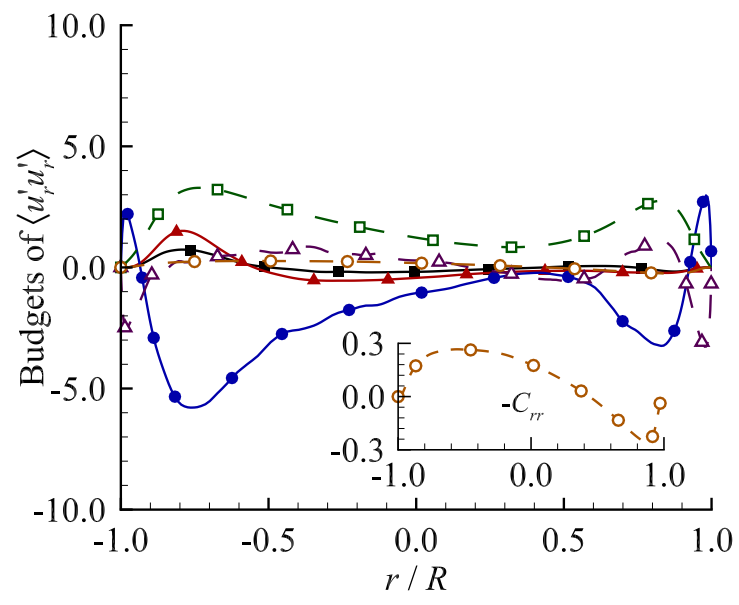

(b) $R o_{\tau}=0.2$

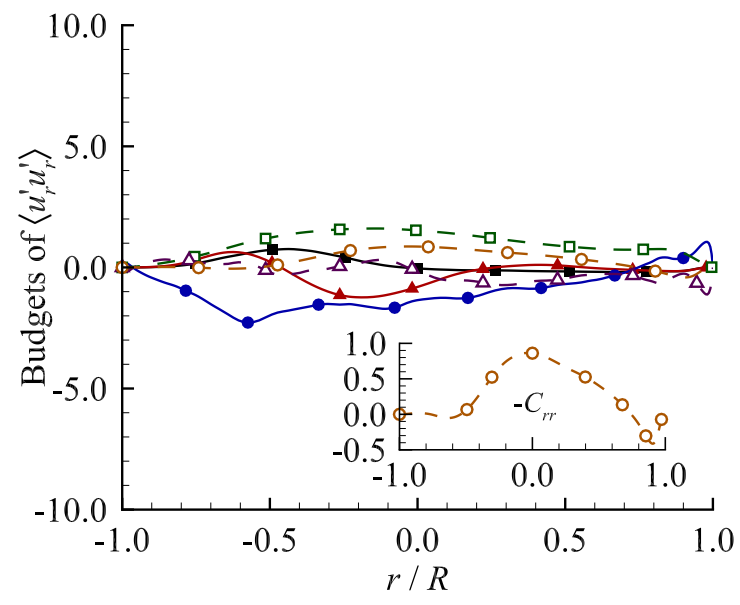

(c) $R o_{\tau}=0.8$

Figure 3.11: Profiles of the budget terms of $\left\langle u_{r}^{\prime} u_{r}^{\prime}\right\rangle$ in the central vertical plane $\left(\theta=90^{\circ}\right)$ of the pipe for different rotation numbers. All budget terms have been non-dimensionalized using $u_{\tau}^{3} / R$.

comparing Figs. 3.12(a), (b) and (c), it becomes clear that in both non-rotating and rotating flow cases, the budget of $\left\langle u_{z}^{\prime} u_{r}^{\prime}\right\rangle$ is primarily balanced by the diffusion term $-D_{z r}$ and pressure-strain term $-\Pi_{z r}$ in the near-wall region. However, at the pipe center, the budget is dominated by the production term $-P_{z r}$ and pressure-strain term $-\Pi_{z r}$, and furthermore, these two terms balance each other so well as if they 


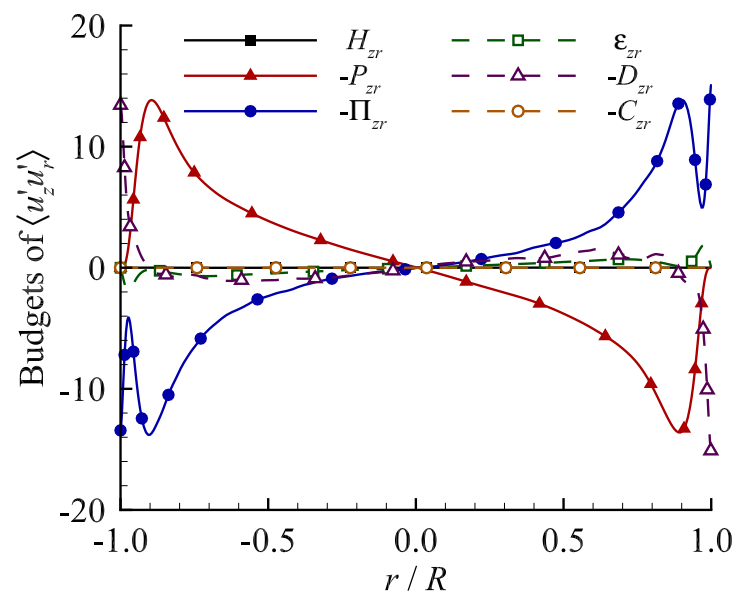

(a) $R o_{\tau}=0.0$

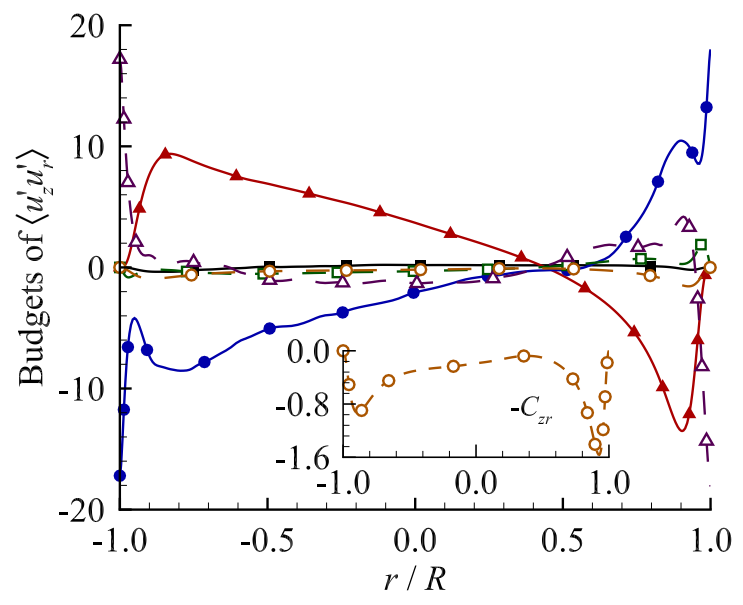

(b) $R o_{\tau}=0.2$

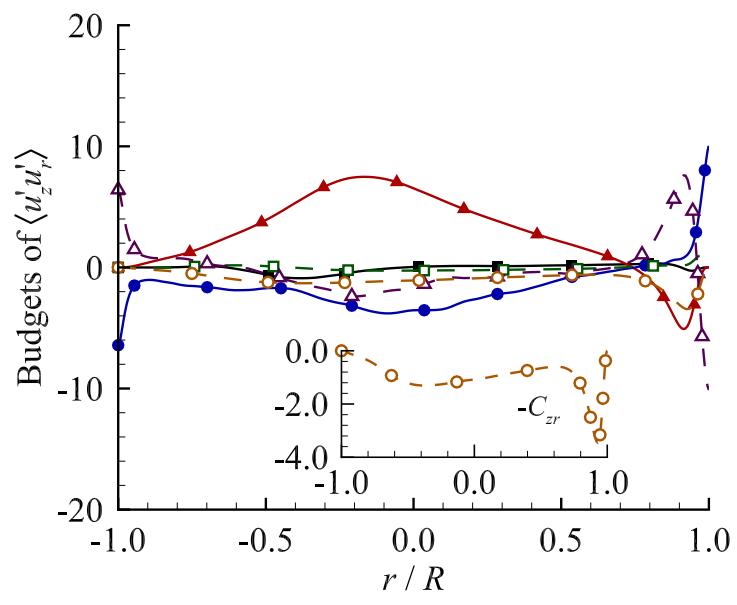

(c) $R o_{\tau}=0.8$

Figure 3.12: Profiles of the budget terms of $\left\langle u_{z}^{\prime} u_{r}^{\prime}\right\rangle$ in the central vertical plane $\left(\theta=90^{\circ}\right)$ of the pipe for different rotation numbers. All budget terms have been non-dimensionalized using $u_{\tau}^{3} / R$.

are a mirror reflection of each other. The Coriolis effect is apparent by comparing Figs. 3.12(a), (b) and (c): for the non-rotating flow case, the profiles of these two dominant terms, $-P_{z r}$ and $-\Pi_{z r}$, are symmetrical in the vertical direction; however, in the rotating flow cases, their profiles become asymmetrical and go across each other at $r / R=0.45$ for $R o_{\tau}=0.2$ and at $r / R=0.74$ for $R o_{\tau}=0.8$, respectively. 


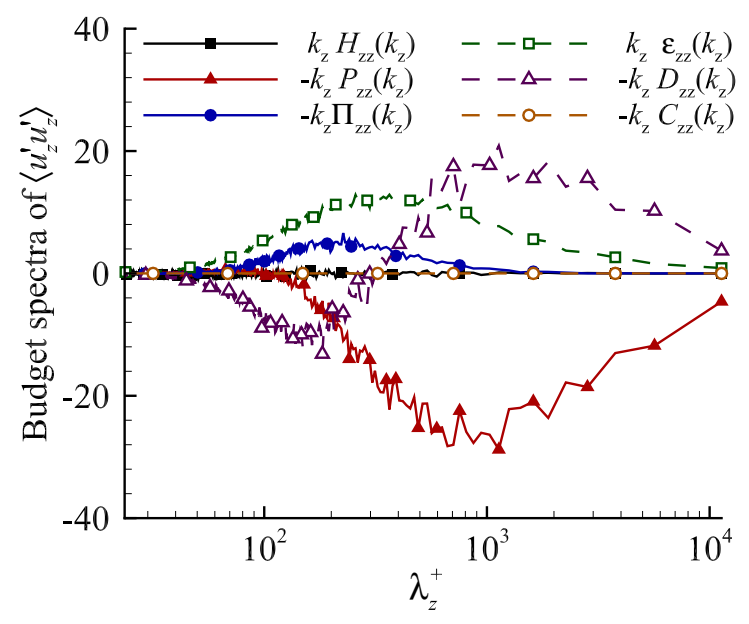

(a) $R o_{\tau}=0.0$

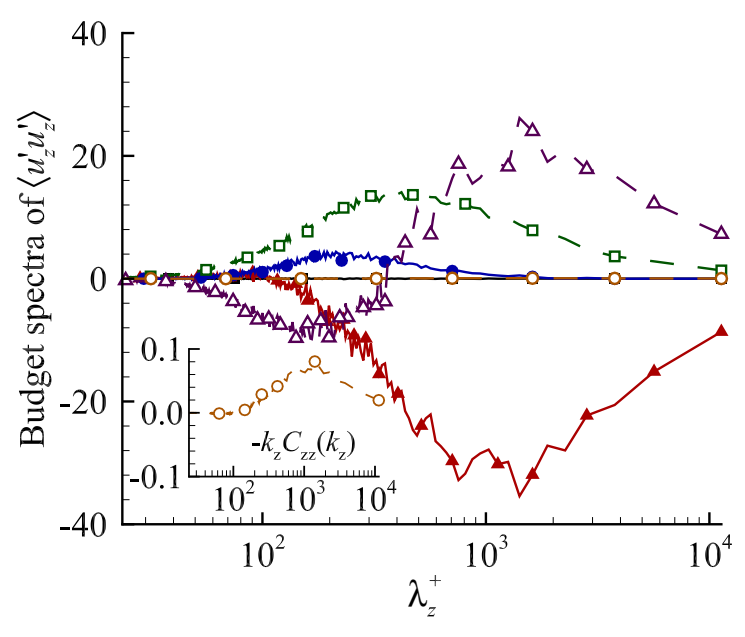

(b) $R o_{\tau}=0.2$

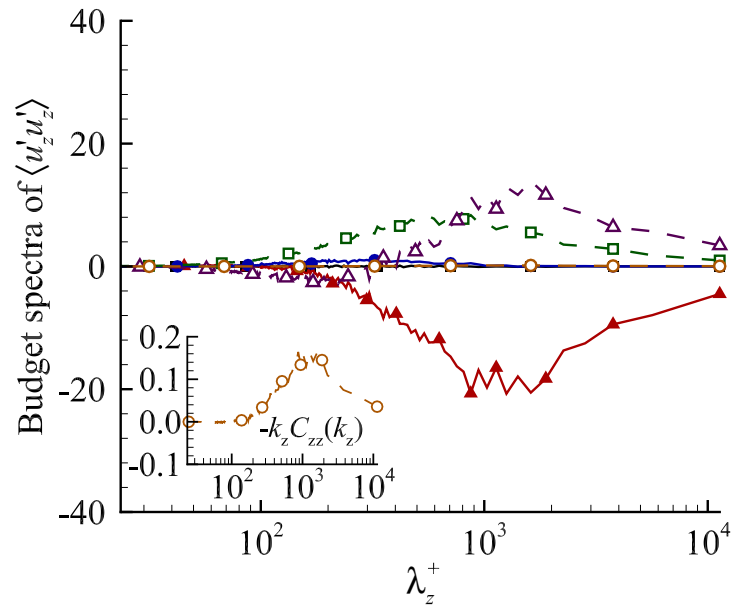

(c) $R o_{\tau}=0.8$

Figure 3.13: Pre-multiplied spectra of the budget terms of $\left\langle u_{z}^{\prime} u_{z}^{\prime}\right\rangle$ with respect to wavelength $\lambda_{z}^{+}$calculated along the axial line of the pipe at wall-normal position $y^{+}=14.24$ on the pressure side $\left(\theta=90^{\circ}\right)$ for $R o_{\tau}=0.0,0.2$ and 0.8. All budget terms have been non-dimensionalized using $u_{\tau}^{3} / R$.

The above analysis of the budget balance of the Reynolds stresses based on Figs. 3.10-3.12 was conducted in the physical space. In order to develop a deeper understanding of the transport process of Reynolds stresses under a radially-rotating condition, it is of research interest to further look into the budget terms in the spectral 
space. Fig. 3.13 compares the pre-multiplied spectra of the budget terms of $\left\langle u_{z}^{\prime} u_{z}^{\prime}\right\rangle$ as a function of wavelength $\lambda_{z}^{+}$at the wall-normal position $y^{+}=14.24$ on the pressure side $\left(\theta=90^{\circ}\right)$ of the pipe under the non-rotating $\left(R o_{\tau}=0.0\right)$ and rotating $\left(R o_{\tau}=0.2\right.$ and 0.8) conditions. As is evident in Fig. 3.13(a), for the non-rotating case, in the inner region near the pressure side of the pipe, the peak value of both the spectra of pressure-strain $-k_{z} \Pi_{z z}\left(k_{z}\right)$ and dissipation $k_{z} \varepsilon_{z z}\left(k_{z}\right)$ terms occurs at small wavelengths (indicated by the wavelength $\lambda_{z}^{+}$). In these wavelengths, the pressure-strain term $-\Pi_{z z}$ transfers energy from $\left\langle u_{z}^{\prime} u_{z}^{\prime}\right\rangle$ to $\left\langle u_{r}^{\prime} u_{r}^{\prime}\right\rangle$ which is consistent with the observation in Figs. 3.10(a) and 3.11(a); and the dissipation term $\varepsilon_{z z}$ supports the energy sinking mechanism. These indicate that both terms promote the turbulence isotropy at small wavelengths. It is interesting to be observed that the spectrum of diffusion term $-k_{z} D_{z z}\left(k_{z}\right)$ has a peak value at small wavelengths, implying that the diffusion term $-D_{z z}$ (contributed by turbulent diffusion term) drains energy through inertia at small wavelengths. Furthermore, the spectra of diffusion $-k_{z} D_{z z}\left(k_{z}\right)$ and production $-k_{z} P_{z z}\left(k_{z}\right)$ terms peaks at large wavelengths around $500<\lambda_{z}^{+}<2000$, demonstrating that the diffusion term $-D_{z z}$, together with dissipation term $\varepsilon_{z z}$, counteracts the energy transferred by the production term $-P_{z z}$ from mean flow to $\left\langle u_{z}^{\prime} u_{z}^{\prime}\right\rangle$. It is also observed that the energy spectrum $k_{z} \phi_{z z}$ and the production spectrum $-k_{z} P_{z z}\left(k_{z}\right)$ are almost synchronized at $500<\lambda_{z}^{+}<2000$; and the peak value of both of them appears at $\lambda_{z}^{+} \approx 1000$. It indicates that comparing with other terms in transport equation of Reynolds stress component $\left\langle u_{z}^{\prime} u_{z}^{\prime}\right\rangle$, the production term has a direct contribution on the increment of the axial turbulence intensity. For the rotating flow, as is shown in Fig. 3.13(b) and (c), the spectrum of Coriolis term $-k_{z} C_{z z}\left(k_{z}\right)$ peaks at large wavelengths; and the Coriolis term, together with the pressure-strain term, transfers energy from $\left\langle u_{z}^{\prime} u_{z}^{\prime}\right\rangle$ to $\left\langle u_{r}^{\prime} u_{r}^{\prime}\right\rangle$. Except the Coriolis term, the mechanism of budget terms involved in transport equation of $\left\langle u_{z}^{\prime} u_{z}^{\prime}\right\rangle$ remains unchanged in the inner region of the pressure side. However, in response to the radial system rota- 
tion, the magnitudes of $-k_{z} P_{z z}\left(k_{z}\right), k_{z} \varepsilon_{z z}\left(k_{z}\right)$ and $-k_{z} D_{z z}\left(k_{z}\right)$ significantly increase at $R o_{\tau}=0.2$, implying that the Coriolis effects result in more turbulence on the flow field. In contrast, as $R o_{\tau}$ reaches 0.8 , the magnitudes of all term spectra become much smaller than those in the non-rotating case, especially at small wavelengths where the values are almost zero. It demonstrates that the turbulent pipe flow starts laminarization at small wavelengths under the radial system rotation.

\subsection{Turbulence structures}

The appearance of the Coriolis forces in a radially-rotating pipe flow drastically alters its dynamics and coherent structures. Induced by the Coriolis force, secondary flows occur in the cross-stream direction, which have been demonstrated using the instantaneous and mean flow fields in Figs. 3.3 and 3.4, respectively. The Coriolis forces also have a significant impact on the development of turbulent flow structures in the axial direction.

Figure 3.14 compares the contours of instantaneous axial vorticity fluctuation $\omega_{z}^{\prime+}$ in a cross-stream plane for the non-rotating case $\left(R o_{\tau}=0.0\right)$ and rotating cases $\left(R o_{\tau}=0.2\right.$ and 0.8), respectively. From Fig. 3.14(a), it is clear that for a non-rotating case, the instantaneous turbulence eddies mostly appear as counter-rotating vortex pairs in the near-wall region and they are uniformly distributed in the circumferential direction. In contrast, as is shown in Fig. 3.14(b) and (c), in the rotating pipe flow cases, most vortices become merged, and the strength of these vortices (as indicted by the contour level of $\omega_{z}^{\prime+}$ ) reduces significantly to reflect the trends of laminarization as $R o_{\tau}$ increases.

Figure 3.15 compares the coherent structures of the non-rotating case $\left(R o_{\tau}=0.0\right)$ and rotating case (with $R o_{\tau}=0.2$ and 0.8), based on the so-called " $\lambda_{c i}$-criterion" [30]. 


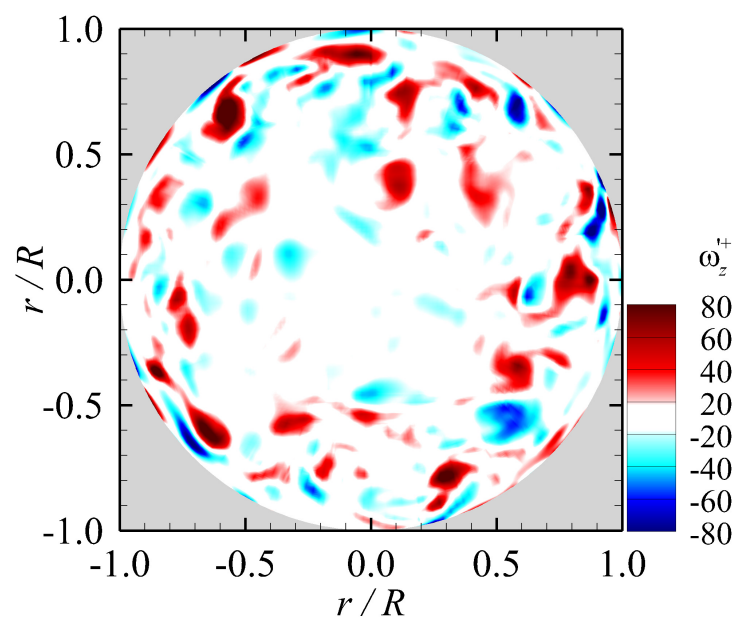

(a) $R o_{\tau}=0.0$

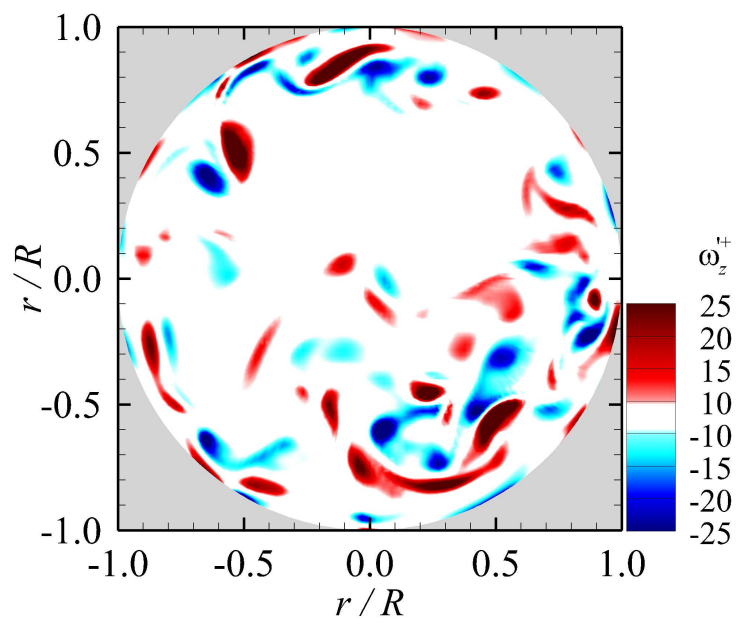

(b) $R o_{\tau}=0.2$

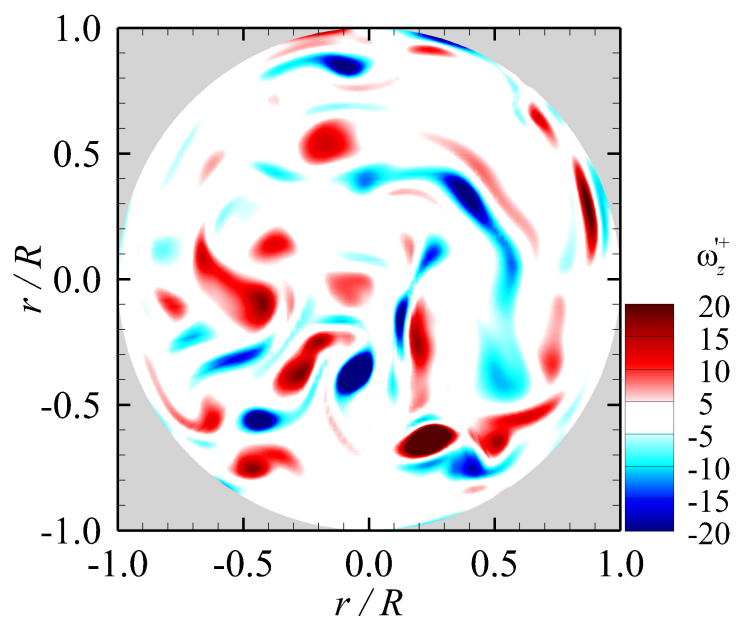

(c) $R o_{\tau}=0.8$

Figure 3.14: Contours of instantaneous axial vorticity fluctuation $\omega_{z}^{\prime+}$ in a crossstream plane at $R o_{\tau}=0.0,0.2$ and 0.8 .

To ensure the visual clarity, only one-fourth axial and one-half cross-stream domain is displayed in the figure. As is evident in Fig. 3.15(a), for the non-rotating case $\left(R o_{\tau}=0.0\right)$, complete hairpin structures are observed. In response to the bursting behaviour, two counter-rotating streamwise vortices form the arch head of a hairpin structure in the outer region through events of ejection (or, the so-called "Q2-events") and sweeping (or, the so-called "Q4-events"). As $R o_{\tau}$ increases to 0.2 as shown in 


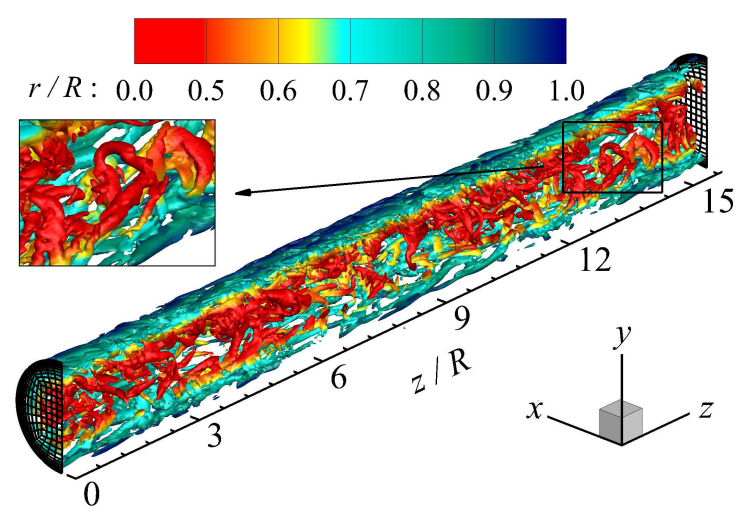

(a) $R o_{\tau}=0.0$

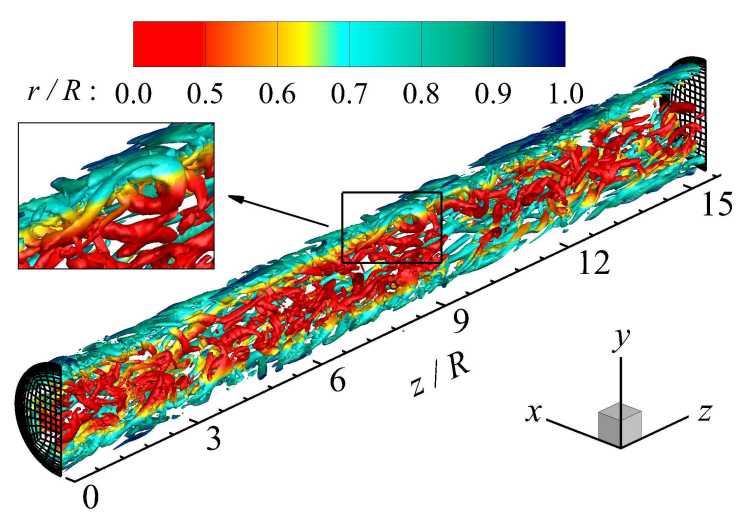

(b) $R o_{\tau}=0.2$

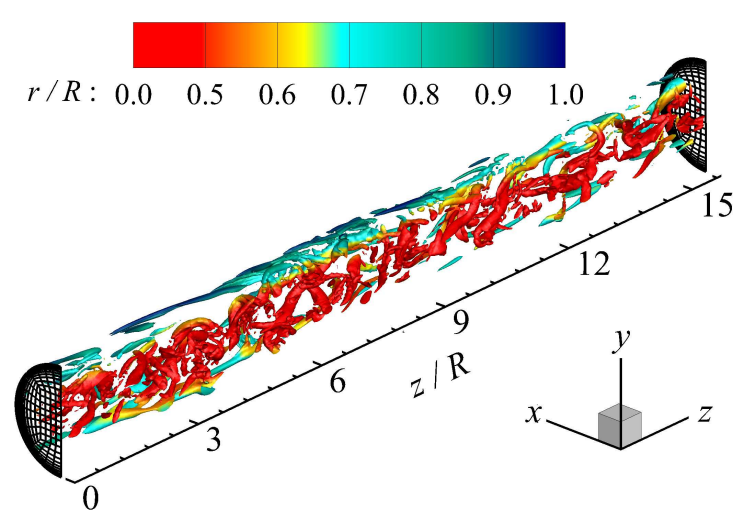

(c) $R o_{\tau}=0.8$

Figure 3.15: Contours of vortical structures for the non-rotating $\left(R o_{\tau}=0.0\right)$ and rotating $\left(R o_{\tau}=0.2\right.$ and 0.8$)$ cases. Only one-fourth axial and one-half cross-stream domain (iso-surfaces are plotted with $\lambda_{c i}=0.8$ ) is displayed. The contours are colored with non-dimensionalized radius $r / R$.

Fig. 3.15(b), complete hairpin structures can still be observed on the pressure side of the pipe because the flow field becomes increasingly turbulent on the pressure side due to the Coriolis effects. Meanwhile, flow structures are suppressed on the suction side of the pipe. However, as shown in Fig. 3.15(c), as the rotation number further increases to $R o_{\tau}=0.8$, complete hairpin vortices can hardly be observed at this high rotation number. In response to radial system rotation, the bursting behaviour is broken preventing the occurrences of Q2- and Q4-events in the near-wall region of 
the pressure side. As a result, the arch heads of the hairpin vortices cannot be formed effectively on the pressure side. On the other hand, the radial system rotation directly accelerates the bursting behaviour on the suction side. Although this acceleration can promote the formation of arch heads directly through the Q2-events of low-momentum streaks, hairpin structures cannot be maintained in this process, which breakdown to finer-scale structures rapidly. By comparing Fig. 3.15(c) with (a) and (b), it is evident that at a high rotation number of $R o_{\tau}=0.8$, the flow becomes less turbulent, and as a result, the amount of flow structures are significantly reduced, especially on the suction side of the pipe.

In the literature, turbulent plane-channel flows subjected to spanwise system rotation have been extensively studied using experimental [29] and numerical [2,3] approaches. It has been concluded that streamwise elgongated Taylor-Görtler-like (TGL) vortices appear in pairs in the cross-stream direction in a spanwise-rotating flow in response to the Coriolis effects. As indicated by Speziale and Thangam [31] and Grundestam et al. [3], the appearance of TGL vortices in a spanwise-rotating channel is triggered by the Coriolis force instability, analogous to the centrifugal instability (associated with surface curvature) that is responsible for the formation of TG vortices in a Taylor-Couette flow. In this research, I am tempted to extend these concepts from the context of spanwise-rotating channel flows to that of radiallyrotating pipe flows, and it would be interesting to find out is TGL vortices exist in a radially-rotating pipe flow. To this purpose, Fig. 3.16 plots the contours of timeaveraged axial fluctuating vorticity $\overline{\omega_{z}^{\prime}}$ at $R o_{\tau}=0.8$. Here, the overbar denotes the time-averaging over a duration of 80 LETOTs (or $80 R / u_{\tau}$ ). To improve the visual clarity, only one-fourth of axial computational domain size is displayed, iso-surfaced by $\left|{\overline{\omega_{z}^{\prime}}}^{+}\right|=0.7$. Clearly, in a rapidly radially-rotating pipe flow, the TGL vortices are present. The contour-rotating roll cells are visualized using the positive and negative values of ${\overline{\omega_{z}^{\prime}}}^{+}$(represented by red and blue colours, respectively). From Fig. 3.16, it 


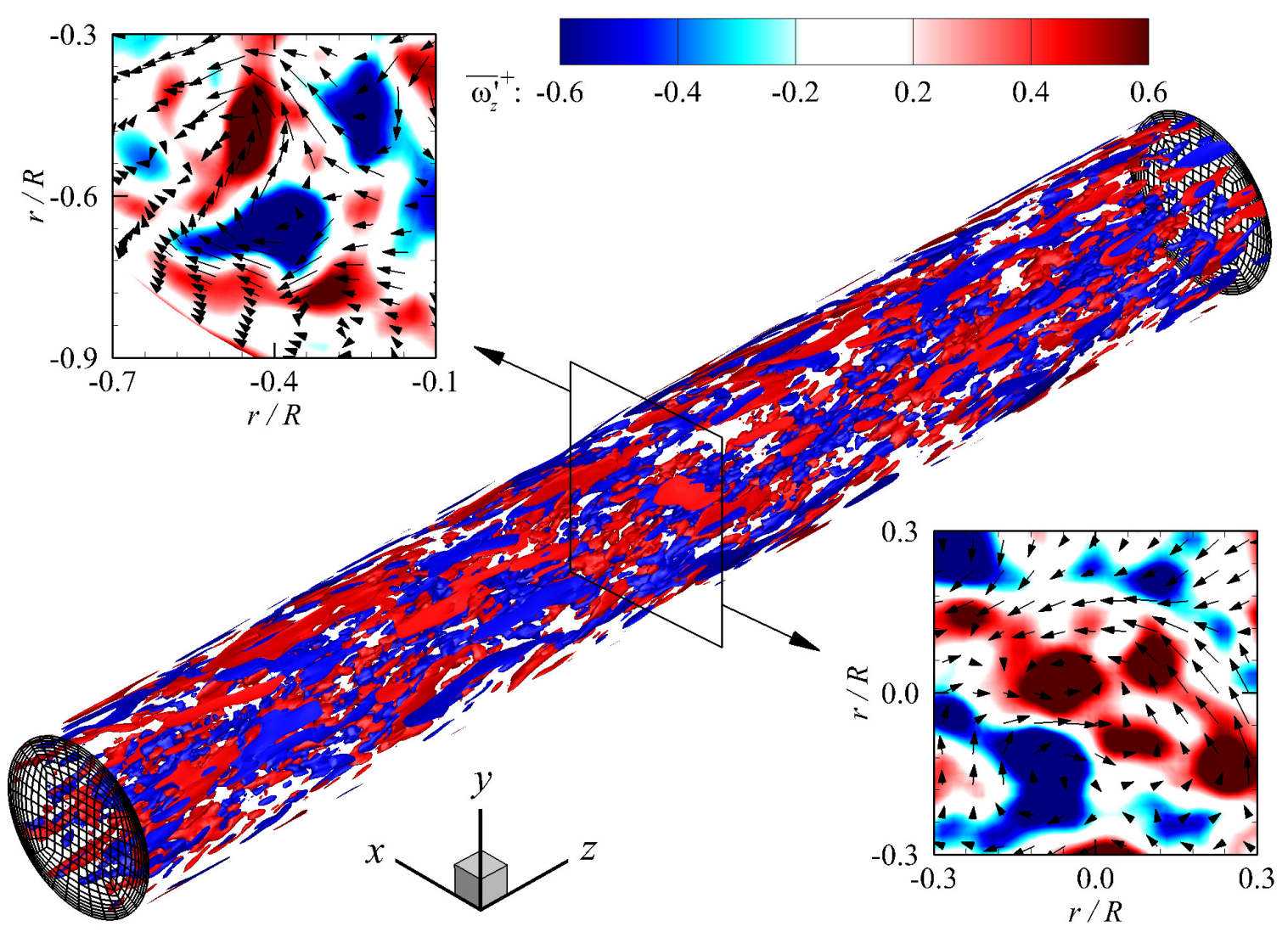

Figure 3.16: Contours of streamwise-elongated Taylor-Görtler vortical structures for the rotating pipe flow $\left(R o_{\tau}=0.8\right)$, based on time-averaged axial vorticity fluctuations ${\overline{\omega_{z}^{\prime}}}^{+}$. Only one-fourth of pipe length (iso-surfaces are plotted with $\left|\overline{\omega_{z}^{\prime}}{ }^{+}\right|=0.7$ ) is displayed. Red and blue colours represent negative and positive values of $\overline{\omega_{z}^{\prime}}+$, respectively. The two partially enlarged panels are extracted to demonstrate the characteristic instantaneous turbulent flow structures in the cross-stream direction, superimposed with time-averaged velocity vectors.

is seen that the TGL vortices are of larger length scales in the axial direction than in the radial and azimuthal directions. 


\section{Chapter 4}

\section{Conclusions and future studies}

In this chapter, major results on DNS of turbulent flow in a circular pipe subjected to radial system rotation are concluded. Future studies are briefly discussed to include passive heat transfer, transport equations of Reynolds stresses in the spectral space and turbulent radially-rotating pipe flow at high Reynolds numbers.

\subsection{Conclusions on DNS of turbulent flow in a cir- cular pipe subjected to radial system rotation}

Fully-developed turbulent flow confined within a circular pipe subjected to radial system rotation has been studied using DNS. A wide range of rotation numbers have been tested, varying from $R o_{\tau}=0.0$ to 1.0. In order to ensure the most energetic large-scale eddy motions to be captured by DNS, the pipe length was set to $L_{z}=$ $20 \pi R$, which represents the longest pipe length in numerical simulation of rotating pipe flows in the current literature.

In response to the radial system rotation imposed, the flow field becomes appar- 
ently asymmetric in the vertical direction. As the rotation number increases from $R o_{\tau}=0.2$ to 1.0 , the pressure and suction sides of the pipe become increasingly dominated by high- and low-speed flows, respectively. Clearly, the role of the Coriolis force is to push the high-momentum fluids towards the pressure side and low-momentum fluids towards the suction side of the pipe. In consequence, as $R o_{\tau}$ increases from 0.0 to 1.0, the magnitude of the skin friction coefficient at the pressure and suction sides of the pipe increases and decreases monotonically, respectively.

For the mean flow field, secondary flows in form of a pair of counter-rotating axial vortices appear in the cross-stream direction in response to the radial system rotation imposed. As $R o_{\tau}$ increases from 0.0 to 0.8 , a quasi Taylor-Proudman region is briefly observed in the pipe center, which features an invariant mean axial velocity distribution along the rotating direction. At the highest rotation number $\left(R o_{\tau}=1.0\right)$ tested, the flow becomes completely laminarized under the influence of the Coriolis force, and two pairs of counter-rotating axial vortices are present in the mean secondary flows.

As soon as the radial system rotation is imposed on the pipe flow, the wellrecognized linear distribution of the Reynolds shear stress $\left\langle u_{z}^{\prime} u_{r}^{\prime}\right\rangle$ characteristic of a non-rotating pipe flow starts to lose effect. As the rotation number increases, the strength of $\left\langle u_{z}^{\prime} u_{r}^{\prime}\right\rangle$ decreases on both sides of the pipe. In the near-wall region of the pressure side of the pipe, the peak values of Reynolds stresses $\left\langle u_{r}^{\prime} u_{r}^{\prime}\right\rangle,\left\langle u_{\theta}^{\prime} u_{\theta}^{\prime}\right\rangle$ and $\left\langle u_{z}^{\prime} u_{r}^{\prime}\right\rangle$ decrease monotonically as the rotation number increases. However, it is interesting to observe that the peak value of the streamwise Reynolds normal stress component $\left\langle u_{z}^{\prime} u_{z}^{\prime}\right\rangle$ occurs at $R o_{\tau}=0.2$ at $y^{+}=14.24$ on the pressure side of the pipe. The pre-multiplied spectrum of the axial velocity fluctuations at this position further shows that as $R o_{\tau}$ increases from 0 to 0.2 , the TKE level as indicated by $k_{z} \phi_{z z}$ increases at large wavelengths. However, once the rotation number increases beyond $R o_{\tau}=0.8$, the TKE level drops dramatically because the effect of the Coriolis forces 
tends to suppress turbulent motions.

Depending on the sign of $\left\langle u_{z}^{\prime} u_{r}^{\prime}\right\rangle$, the Coriolis force shifts TKE between two Reynolds normal stress components $\left\langle u_{z}^{\prime} u_{z}^{\prime}\right\rangle$ and $\left\langle u_{r}^{\prime} u_{r}^{\prime}\right\rangle$, simply because $C_{z z}=-C_{r r}$. Also, if there is a net energy balance between the $\left\langle u_{z}^{\prime} u_{z}^{\prime}\right\rangle$ and $\left\langle u_{r}^{\prime} u_{r}^{\prime}\right\rangle$, the Coriolis force shifts this amount of energy balance $\left(\left\langle u_{z}^{\prime} u_{z}^{\prime}\right\rangle-\left\langle u_{r}^{\prime} u_{r}^{\prime}\right\rangle\right)$ to the Reynolds shear stress component $\left\langle u_{z}^{\prime} u_{r}^{\prime}\right\rangle$. At the pipe center, the budget of the Reynolds shear stress $\left\langle u_{z}^{\prime} u_{r}^{\prime}\right\rangle$ is dominated by the production term $-P_{z r}$ and pressure-strain term $-\Pi_{z r}$, they balance as if they are a mirror reflection of each other. Furthermore, owing to the presence of the Coriolis force, the profiles of $-P_{z r}$ and $-\Pi_{z r}$ become asymmetrical about the pipe center and their crossing point is shifted towards the pressure side of the pipe, a feature that is in sharp contrast to that of a non-rotating pipe flow. It is clear that the mechanism of budget terms of $\left\langle u_{z}^{\prime} u_{z}^{\prime}\right\rangle$ is invariant in the inner region of the pressure side for all wavelengths; and the values of budget spectra decrease as rotation number increases from $R o_{\tau}=0.2$. On the one hand, the dissipation $\varepsilon_{z z}$ and pressure-strain $-\Pi_{z z}$ terms promote the turbulent isotropy at small wavelengths. On the other hand, the diffusion $-D_{z z}$, Coriolis production $-C_{z z}$ and production $-P_{z z}$ terms, together with $\varepsilon_{z z}$, maintain the budget balance at large wavelengths. In addition, It is also interesting to be observed that the turbulent pipe flow starts the laminrization from small wavelengths.

For the non-rotating $\left(R o_{\tau}=0.0\right)$ and rotating flows at a low rotation number $\left(R o_{\tau}=0.2\right)$, complete hairpin structures are observed. In response to the bursting behaviour, two counter-rotating streamwise vortices form the arch head of a hairpin structure in the outer region through ejection and sweep events along the curved pipe wall. However, as the rotation number further increases to $R o_{\tau}=0.8$, the bursting behaviour is broken preventing the occurrences of ejection and sweep events in the near-wall region of the pressure side; as a result, the arch heads of the hairpin structures cannot be formed effectively. On the other hand, the radial system rotation 
directly accelerates the bursting behaviour on the suction side. Although this acceleration can promote the formation of arch heads directly through the ejection events of low-momentum streaks, hairpin structures cannot be maintained in this process, and breakdown to finer-scale structures rapidly. At a high rotation number of $R_{o_{\tau}}=0.8$, the flow becomes less turbulence, and as a result, the amount of flow structures are significantly reduced, especially on the suction side of the pipe. From this study, it is confirmed that TGL vortices exist in radially-rotating pipe flows, which are larger in the axial direction than in the radial and azimuthal directions.

\subsection{Future studies}

Three interesting aspects can be further extended from this thesis in the future studies, which are:

- turbulent heat transfer of radially-rotating circular pipe flows. An uniform peripheral wall heat flux boundary condition can be added to the spectral-element code. Through the same mesh and flow conditions, temperature fields will be conducted to post-process two-point auto-correlation and pre-multiplied spectra of temperature fluctuations, followed by instantaneous and mean temperature fields, Nusselt number, first- and second-order thermal statistics, and transport equation of heat fluxes. This research will focus on the effects of Coriolis force on thermal convention in a turbulent circular pipe flow with respect to different rotation numbers.

- transport of velocity-spectrum tensor in radially-rotating turbulent pipe flows. Specifically, this research will mainly focus on the budget balance of Reynolds stresses in the spectral space. In order to analyze the budget terms, three transport equations of Reynolds stresses (i.e., $\left\langle u_{z}^{\prime} u_{z}^{\prime}\right\rangle,\left\langle u_{r}^{\prime} u_{r}^{\prime}\right\rangle$ and $\left.\left\langle u_{z}^{\prime} u_{r}^{\prime}\right\rangle\right)$ can be 
analyzed in the spectral space using the current statistical results. Therefore, the effect of Coriolis forces on the occurrence of secondary flow and on the transport of Reynolds stresses in a radially-rotating turbulent pipe flow can be systemically investigated in spectral space.

- turbulent radially-rotating pipe flow at high Reynolds numbers. This research will investigate the effects of Coriolis forces on the high-Reynolds-number turbulent pipe flows in comparison with the flows at $R e_{\tau}=180$. In order to conduct a thorough comparative study, all simulations will be based on the same mesh and boundary conditions; and two Reynolds numbers will be considered to perform DNS, which are $R e_{\tau}=395$ and 610 . The similarity and difference between turbulent radially-rotating pipe flows at different Reynolds numbers will be investigated following a similar procedure used in this thesis.

To ensure that these three goals can be achieved, the spectral-element code "Semtex" has to be further developed and modified to satisfy my requirements on DNS. For instance, I will need to add an uniform peripheral wall heat flux boundary condition into the code in order to conduct turbulent heat transfer research. Furthermore, I will also need to develop the post-process code in order to explore more interesting flow mechanisms and dynamics. Two major issues have to be concerned in order to conduct these studies. First, the computational resources could be so limited and expensive. I will have to efficiently allocate the computational resources on simulations. Moreover, the space of data storage can be another problem. In the current thesis, data in each simulation takes up to 310 GB. For example, if I simulate one case at $R e_{\tau}=395$, I would need another 1TB storage space to store its instantaneous 3 -D velocity and temperature fields. Therefore, in the future, how to reasonably use computational resources and to improve storage efficiency will be very important in order to successfully conduct these studies. 


\section{References}

[1] L. Belhoucine, M. Deville, A. R. Elazehari, and M. O. Bensalah, "Explicit algebraic reynolds stress model of incompressible turbulent flow in rotating square duct," Comput. Fluids, vol. 33, pp. 179-199, 2004.

[2] R. Kristoffersen and H. I. Andersson, "Direct numerical simulation of lowreynolds-number turbulent flow in a rotating channel," J. Fluid Mech., vol. 256, pp. 163-197, 1993.

[3] O. Grundestam, S. Wallin, and A. V. Johansson, "Direct numerical simulation of rotating turbulent channel flow," J. Fluid Mech., vol. 598, pp. 177-199, 2008.

[4] S. Wallin, O. Grundestam, and A. V. Johansson, "Laminarization mechanisms and extreme-amplitude states in rapidly rotating plane channel flow," J. Fluid Mech., vol. 730, pp. 193-219, 2013.

[5] Z.-H. Xia, Y.-P. Shi, and S.-Y. Chen, "Direct numerical simulation of turbulent channel flow with spanwise rotation," J. Fluid Mech., vol. 788, pp. 42-56, 2016.

[6] J. Pallares and L. Davidson, "Large-eddy simulations of turbulent flow in a rotating square duct," Phys. Fluids, vol. 12, no. 11, pp. 2878-2894, 2000. 
REFERENCES

[7] Y.-J. Dai, W.-X. Huang, C.-X. Xu, and G.-X. Cui, "Direct numerical simulation of turbulent flow in a rotating square duct," Phys. Fluids, vol. 27, p. 065104, 2015 .

[8] X.-J. Fang, Z.-X. Yang, B.-C. Wang, and D. J. Bergstrom, "Direct numerical simulation of turbulent flow in a spanwise rotating square duct at high rotation numbers," Int. J. Heat Fluid Flow, vol. 63, pp. 88-98, 2017.

[9] J. Eggels, F. Unger, M. Weiss, J. Westerweel, R. Adrian, R. Friedrich, and F. Nieuwstadt, "Fully developed turbulent pipe flow: A comparison between direct numerical simulation and experiment," J. Fluid Mech., vol. 268, pp. 175210, 1994.

[10] X.-H. Wu and P. Moin, "A direct numerical simulation study on the mean velocity characteristics in turbulent pipe flow," J. Fluid Mech., vol. 608, pp. 81-112, 2008 .

[11] C. Chin, A. S. H. Ooi, I. Marusic, and H. M. Blackburn, "The influence of pipe length on turbulence statistics computed from direct numerical simulation data," Phys. Fluids, vol. 22, p. 115107, 2010.

[12] X.-H. Wu, J. R. Baltzer, and R. J. Adrian, "Direct numerical simulation of a 30R long turbulent pipe flow at $R^{+}=685$ : large- and very large-scale motions," J. Fluid Mech., vol. 698, pp. 235-281, 2012.

[13] S. N. Barua, "Secondary flow in a rotating straight pipe," Proc. R. Soc. Lond. A, vol. 227, pp. 133-139, 1954.

[14] H. Ishigaki, "Analogy between laminar flows in curved pipes and orthogonally rotating pipes," J. Fluid Mech., vol. 268, pp. 133-145, 1994. 
REFERENCES

[15] H. Ishigaki, "Analogy between turbulent flows in curved pipes and orthogonally rotating pipes," J. Fluid Mech., vol. 307, pp. 1-10, 1996.

[16] U. Lei, M. J. Lin, H. J. Sheen, and C. M. Lin, "Velocity measurements of the laminar flow through a rotating straight pipe," Phys. Fluids, vol. 6, no. 6, pp. 1972 1982, 1994.

[17] H. M. Blackburn and S. J. Sherwin, "Formulation of a galerkin spectral elementFourier method for three-dimensional incompressible flows in cylindrical geometries," J. Comp. Phys., vol. 197, pp. 759-778, 2004.

[18] G. E. Karniadakis and S. J. Sherwin, Spectral/hp Element Methods for Computational Fluid Dynamics. New York. United States: Oxford University Press, 2 ed., 2005.

[19] R. K. Sharma and K. Nandakumar, "Multiple, two-dimensional solutions in a rotating straight pipe," Phys. Fluids, vol. 7, no. 7, pp. 1568-1575, 1995.

[20] G. K. El Khoury, P. Schlatter, A. Noorani, P. F. Fischer, G. Brethouwer, and A. V. Johansson, "Direct numerical simulation of turbulent pipe flow at moderately high reynolds numbers," Flow, Turbul. Combust., vol. 91, no. 3, pp. 475$495,2013$.

[21] M. Di Liberto, I. Di Piazza, and M. Ciofalo, "Turbulence structure and budgets in curved pipes," Comput. Fluids, vol. 88, pp. 452-472, 2013.

[22] A. Bolis, C. D. Cantwell, D. Moxey, D. Serson, and S. J. Sherwin, "An adaptable parallel algorithm for the direct numerical simulation of incompressible turbulent flows using a fourier spectral/hp element method and mpi virtual topologies," Comput. Phys. Commun., vol. 206, pp. 17-25, 2016. 
REFERENCES

[23] X.-J. Fang and B.-C. Wang, "On the turbulent heat transfer in a square duct subjected to spanwise system rotation," Int. J. Heat Fluid Flow, vol. 71, pp. 220$230,2018$.

[24] C. Wagner, T. J. Hüttl, and R. Friedrich, "Low-reynolds-number effects derived from direct numerical simulation of turbulent pipe flow," Comput. Fluids, vol. 30, pp. 581-590, 2001.

[25] C. Chin, J. P. Monty, and A. Ooi, "Reynolds number effects in dns of pipe flow and comparison with channels and boundary layers," Int. J. Heat Fluid Flow, vol. 45, pp. 33-40, 2014.

[26] K. C. Kim and R. J. Adrian, "Very large-scale motion in the outer layer," Phys. Fluids, vol. 11, no. 2, pp. 417-422, 1999.

[27] J. Kim, P. Moin, and R. Moser, "Turbulence statistics in fully developed channel flow at low reynolds number," J. Fluid Mech., vol. 177, pp. 133-166, 1987.

[28] Z. Yang and B.-C. Wang, "Capturing taylor-görtler vortices in a streamwiserotating channel at very high rotation numbers," J. Fluid Mech., vol. 838, pp. 658-689, 2018.

[29] J. P. Johnston, R. M. Halleen, and D. K. Lezius, "Effects of spanwise rotation on the structure of two-dimensional fully developed turbulent channel flow," $J$. Fluid Mech., vol. 56, no. 3, pp. 533-557, 1972.

[30] J. Zhou, R. J. Adrian, S. Balachandar, and T. M. Kendall, "Mechanisms for generating coherent packets of hairpin vortices in channel flow," J. Fluid Mech., vol. 387, pp. 353-396, 1999.

[31] C. G. Speziale, "Numerical study of viscous flow in rotating rectangular ducts," J. Fluid Mech., vol. 122, pp. 251-271, 1982. 
[32] T. A. Zang, "On the rotation and skew-symmetric forms for incompressible flow simulations," Appl. Numer. Math., vol. 7, no. 1, pp. 27-40, 1991. 


\section{Appendix A}

\section{Spectral-Element Method}

In this thesis, all direct numerical simulations (DNS) are conducted using a spectralelement method (SEM) code "Semtex" primarily written with $\mathrm{C}++$ and FORTRAN programming languages. The SEM code is based on a high-order finite element technique and is capable of offering geometric flexibility of finite elements while maintaining a high spectral accuracy. Prior to the use of the code, the underlying algorithm needs to be studied. After getting familiar with Semtex and its mesh requirements, I developed a quadrilateral-structural grid generator with MATLAB using Transfinite Interpolation (TFI) method in order to deal with the circular pipe geometry. In simulations, the function of Semtex is to conduct the instantaneous files containing instantaneous flow field in a binary form. Therefore, to ensure that the instantaneous flow field data can be used in post-processing, several standalone post-processing codes have been developed using the $\mathrm{C}++$ and Linux scripting programming languages to calculate turbulence statistics of instantaneous flows, including the mean velocity field, turbulence intensities (RMS), two point auto-correlation coefficients, pre-multiplied energy spectra, TKE, Reynolds stresses, and $\lambda_{c i}$ criterion for flow structure visualizations. Furthermore, the budget terms of transport equations in 
both physical and spectral space for the Reynolds stresses need to be calculated. This appendix describes the temporal and spatial discretizations in details for Semtex code.

\section{A.1 Time-splitting Algorithm}

The governing equations containing the continuity and incompressible Navier-Stokes equations can be written as

$$
\begin{gathered}
\nabla \cdot \mathbf{u}=0 \\
\frac{\partial \mathbf{u}}{\partial t}+\mathbf{N}(\mathbf{u})=-\frac{1}{\rho} \nabla p+\nu \nabla^{2} \mathbf{u}+\mathbf{f}
\end{gathered}
$$

where $\mathbf{N}(\mathbf{u})$ is the nonlinear convective term and can be represented in several different forms as follows

$$
\begin{gathered}
\mathbf{N}(\mathbf{u})=\mathbf{u} \cdot \nabla \mathbf{u} \\
\mathbf{N}(\mathbf{u})=\nabla \cdot(\mathbf{u u}) \\
\mathbf{N}(\mathbf{u})=\frac{1}{2}[\mathbf{u} \cdot \nabla \mathbf{u}+\nabla \cdot(\mathbf{u u})], \\
\mathbf{N}(\mathbf{u})=(\nabla \times \mathbf{u}) \times \mathbf{u}+\frac{1}{2} \nabla(\mathbf{u} \cdot \mathbf{u}),
\end{gathered}
$$

which in order are the convective, divergence, skew-symmetric and rotational forms. Although those forms of $\mathbf{N}(\mathbf{u})$ are identical in the continuous mathematics, they possess significantly different properties after discretization. For a low-order spatial scheme, such as the finite volume (FV) and finite difference (FD) methods, the divergence form is widely used to satisfy the momentum conservation. For a spectral accurate spatial scheme, all four schemes can conduct almost identical results after employing the de-aliasing technique. Nevertheless, de-aliasing can be expensive to implement and is commonly deactivated for the fine enough grid resolution. In com- 
parison with other forms of the nonlinear term, although the skew-symmetric form is more expensive in computation, it is also more tolerable in deal with the aliasing error. In order to maintain a balance between stability and computational cost, the SEM code in this thesis employed the convective and divergence forms to calculate the nonlinear term alternatively [32].

A "stiffly stable" integrating method used for the temporal discretization reads

$$
\frac{\partial \mathbf{u}^{(n+1)}}{\partial t}=\frac{1}{\Delta t} \sum_{q=0}^{J_{e}} \alpha_{q} \mathbf{u}^{(n+1-q)}
$$

where $\alpha_{q}$ are some chosen weights for $J_{e}$-order scheme. In this method, the region of stability shrinks as $J_{e}$ increases. More specifically, Eq. (A.7) represents backward Euler equation at $J_{e}=1$. As $J_{e}$ reaches 2 , it is knows as A-stable method; and it has a good balance between CFL stability and spatio-temporal accuracy.

Three sub-steps of a time-splitting scheme for decoupling the velocity and pressure can be read as follows

$$
\begin{gathered}
\frac{\mathbf{u}^{*}-\mathbf{u}^{n}}{\Delta t}=\sum_{q=0}^{J_{e}-1} \beta_{q}\left[\mathbf{N}\left(\mathbf{u}^{n-q}\right)+\mathbf{f}^{n-q}\right], \\
\frac{\mathbf{u}^{* *}-\mathbf{u}^{*}}{\Delta t}=-\frac{1}{\rho} \nabla p^{n+1} \\
\frac{\alpha_{0} \mathbf{u}^{n+1}-\mathbf{u}^{* *}}{\Delta t}=\nu \nabla^{2} \mathbf{u}^{n+1}
\end{gathered}
$$

where $\beta_{q}$ are some chosen weights for $J_{e}$-order scheme. In Eqs. (A.8) and (A.9), $\mathbf{u}^{*}$ and $\mathbf{u}^{* *}$ are defined as intermediate velocity fields. No boundary conditions are employed in the first sub-step. To ensure that $\mathbf{u}^{* *}$ satisfies the incompressible constraint in the 
second sub-step, it has to be solved by following equation

$$
\frac{1}{\rho} \nabla^{2} p^{n+1}=\frac{\nabla \cdot \mathbf{u}^{*}}{\Delta t}
$$

with subjected velocity boundary condition

$$
\frac{1}{\rho} \frac{\partial p^{n+1}}{\partial n}=\mathbf{n} \cdot \sum_{q=0}^{J_{e}-1} \beta_{q}\left[\mathbf{N}\left(\mathbf{u}^{n-q}\right)-\nu \nabla \times\left(\nabla \times \mathbf{u}^{n-q}\right)\right] .
$$

For the third sub-step as Eq. (A.10), the prescribed boundary conditions are imposed.

\section{A.2 Spatial discretization}

\section{A.2.1 Weighted residual method}

In a 3D simulation, Semtex transforms all physical quantities into a Fourier space in $z$-direction (assumed as homogenous direction). Meanwhile, it interpolates the quantities with the quadrilateral spectral-element method based on Gauss-LobattoLegendre (GLL) Lagrange polynomial in $x$ - and $y$-directions. Semtex also uses the scheme called "iso-parametric projection" to map every quadrilateral element into the canonical domain $(\xi, \gamma) \in[-1,1] \times[-1,1]$. Therefore, Eqs. (A.11) and (A.10) can be further expressed respectively as

$$
\begin{aligned}
& \frac{1}{\rho}\left[\nabla_{x y}^{2} \hat{p}^{n+1}-k_{z}^{2} \hat{p}^{n+1}\right]=\nabla \cdot\left(\frac{\hat{\mathbf{u}}^{*}}{\Delta t}\right), \\
& \nabla_{x y}^{2} \hat{\mathbf{u}}^{n+1}-\left(\frac{1}{\nu \Delta t}+k_{z}^{2}\right) \hat{\mathbf{u}}^{n+1}=-\frac{\hat{\mathbf{u}}^{* *}}{\nu \Delta t},
\end{aligned}
$$

where $\nabla_{x y}^{2}=\partial^{2} / \partial x^{2}+\partial^{2} / \partial y^{2}$ is the Laplace operator in cross-stream directions, $k_{z}$ denotes the wavenumber in $z$-direction and $(\hat{)})$ represents the quantities in Fourier 
space.

In order to simplify above Eqs. (A.14) and (A.13), a two-dimensional Helmholtz equation can be employed in general form as

$$
\nabla^{2} u-\lambda^{2} u=f
$$

where $\lambda$ and $f$ represent real and general constants, respectively. The subscript in $\nabla_{x y}$ is omitted for convenience. In a bounded domain of $\Omega$, Eq. (A.15) is required to be integrated and multiplied by a weight function $v$, i.e.,

$$
\int_{\Omega}\left(\nabla^{2} u-\lambda^{2} u-f\right) v d \Omega=0
$$

The above equation can be further expressed using the integration by parts (IBP) on $\nabla^{2}$ as

$$
\int_{\Omega} \nabla u \nabla v d \Omega+\lambda^{2} \int_{\Omega} u v d \Omega=-\int_{\Omega} f v d \Omega+\oint_{\partial \Omega} h v d(\partial \Omega)
$$

where $h$ represents the Neumann boundary condition defined as $\mathbf{n} \cdot \nabla \mathbf{u}$. In order to partition the whole bounded domain $\Omega$ into sub-domain $\Omega_{e}$ for each element, a mathematical definition has to be introduced, which is a integral of sum equals a sum of integral as $\int_{\Omega}(\cdot) d \Omega=\sum_{e=1}^{N_{e}} \int_{\Omega_{e}}(\cdot) d \Omega_{e}$. Therefore, the above equation can be rearranged as

$$
\sum_{e=1}^{N_{e}}\left[\int_{\Omega_{e}} \nabla u \nabla v d \Omega_{e}+\lambda^{2} \int_{\Omega_{e}} u v d \Omega_{e}+\int_{\Omega_{e}} f v d \Omega_{e}-\oint_{\partial \Omega_{e}} h v d\left(\partial \Omega_{e}\right)\right]=0,
$$

where $N_{e}$ denotes the total number of elements. 
APPENDIX A. SPECTRAL-ELEMENT METHOD

\section{A.2.2 Discretization in a $1 \mathrm{D}$ quadrilateral element}

To ensure that each of the integrations of sub-domains in Eq. (A.18) can be discretized, some important concepts in 1D SEM have to be introduced.

A continuous base function $u(\xi) \in[-1,1]$ in a variational form reads as

$$
u(\xi)=\sum_{i=0}^{N} u_{i} \Psi_{i}(\xi)
$$

where $\Psi_{i}(\xi)$ is defined as the nodal base functions and $u_{i}$ is the coefficients. In the canonical domain, the nodal base function is chosen as Lagrange polynomial satisfying $\Psi_{i}\left(\xi_{j}\right)=\delta_{i j}$ for $\{j=0,1, \cdots, N\}$ where $\xi_{j}$ is the GLL quadrature points, corresponding to the zeros of $\left(1-\xi^{2}\right) L_{N}^{\prime}(\xi)$ with $L_{N}^{\prime}(\xi)$ being the Legendre polynomial of order $N$.

Because any linear operation on continuous base function $u(\xi)$ in Eq. (A.19) can be discretized, the numerical integration on $u(\xi)$ with the Gaussian quadrature rule reads

$$
\int_{-1}^{1} u(\xi) d \xi=\sum_{i=0}^{N} u_{i} \int_{-1}^{1} \Psi_{i}(\xi) d \xi=\sum_{i=0}^{N} w_{i} u_{i}=\hat{\mathbf{w}}^{T} \mathbf{u}
$$

where the quadrature weight for the $i$-th base function is defined as $w_{i}=\int_{-1}^{1} \Psi_{i}(\xi) d \xi$. To simplify the above equation, matrices $\hat{\mathbf{w}}=\left[w_{0}, \cdots, w_{N}\right]^{T}$ and $\mathbf{u}=\left[u_{0}, \cdots, u_{N}\right]^{T}$ are introduced.

The spatial derivative on $u\left(\xi_{j}\right)$ reads

$$
\frac{\partial u\left(\xi_{j}\right)}{\partial \xi}=\sum_{i=0}^{N} u_{i} \frac{\partial \Psi_{i}\left(\xi_{j}\right)}{\partial \xi}=\hat{\mathbf{D}} \mathbf{u}
$$

where $\hat{\mathbf{D}}=\left[\partial \Psi_{i}\left(\xi_{j}\right) / \partial \xi\right]$ represents the differentiation matrix.

In order to employ Galerkin weighted residual method, the nodal base function 
has also been used to represent the weight function, i.e., $v_{j}=\Psi_{j}(\xi)$. As such,

$$
\left(u, v_{j}\right)=\sum_{i=0}^{N} u_{i} \int_{-1}^{1} \Psi_{i}(\xi) \Psi_{j}(\xi) d \xi
$$

By using the Gaussian quadrature rule and $\Psi_{i}\left(\xi_{j}\right)=\delta_{i j}$, which can also be expressed as

$$
\left(u, v_{j}\right)=\sum_{i=0}^{N} u_{i} \sum_{k=0}^{N} w_{k} \Psi_{i}\left(\xi_{k}\right) \Psi_{j}\left(\xi_{k}\right)=\sum_{i=0}^{N} u_{i} \sum_{k=0}^{N} w_{k} \delta_{i k} \delta_{j k}=u_{j} w_{j}=\mathbf{v}_{j}^{T} \hat{\mathbf{W}} \mathbf{u}
$$

Here, the mass matrix is defined as $\hat{\mathbf{W}}=\hat{\mathbf{I}} \hat{\mathbf{w}}$ where $\hat{\mathbf{I}}$ is the identical matrix of rank $N+1 . \mathbf{v}_{j}$ is the $j$-th weight function in the variational form of Eq. (A.19). Because only the $j$-th row is identical in $\mathbf{v}_{j}$, i.e., $\mathbf{v}_{j}=[0, \cdots, 1, \cdots, 0]^{T}$. Therefore, Eq. (A.22) can be further expanded for all weight functions with a simplified matrix notation as

$$
(u, v)=\hat{\mathbf{W}} \mathbf{u}
$$

where $\hat{\mathbf{I}}$ is related with the weight functions as $\hat{\mathbf{I}}=\left[\hat{\mathbf{v}}_{0}, \cdots, \hat{\mathbf{v}}_{N}\right]^{T}$.

Moreover, in order to discretize $(\nabla u, \nabla v)$ of Eq. (A.18) in a $1 \mathrm{D}$ quadrilateral element, $(\nabla u, \nabla v)$ can be expressed as

$$
\left(\frac{\partial u}{\partial \xi}, \frac{\partial v_{j}}{\partial \xi}\right)=\left(\hat{\mathbf{D}} \mathbf{u}, \hat{\mathbf{D}} \mathbf{v}_{j}\right)=\left(\hat{\mathbf{D}} \mathbf{v}_{j}\right)^{T} \hat{\mathbf{W}} \hat{\mathbf{D}} \mathbf{u}=\mathbf{v}_{j}^{T} \hat{\mathbf{D}}^{T} \hat{\mathbf{W}} \hat{\mathbf{D}} \mathbf{u}
$$

Thus, for all weight function, the discretization of $(\nabla u, \nabla v)$ can be further derived as follows,

$$
\left(\frac{\partial u}{\partial \xi}, \frac{\partial v}{\partial \xi}\right)=\hat{\mathbf{D}}^{T} \hat{\mathbf{W}} \hat{\mathbf{D}} \mathbf{u}
$$


Meanwhile, the last term in Eq. (A.18) in 1D quadrilateral element can be derived as

$$
\int_{\partial \Omega_{e}} h v_{j} d \tau=\int_{-1}^{1} h v_{j} \frac{\partial \tau}{\partial \xi} d \xi=\sum_{i=0}^{N} w_{i} h_{i} \Psi_{j}\left(\xi_{i}\right) \frac{\partial \tau}{\partial \xi}\left(\xi_{i}\right)=w_{j} h_{j} \frac{\partial \tau}{\partial \xi}\left(\xi_{j}\right)
$$

where $\tau$ denotes the tangential direction. Therefore, for all weight functions, the last component of Eq. (A.18) reads

$$
\int_{\partial \Omega_{e}} h v d \tau=\mathbf{B h}
$$

where $\mathbf{B}=\operatorname{diag}\left(w_{0} \frac{\partial \tau}{\partial \xi}\left(\xi_{0}\right), \cdots, w_{N} \frac{\partial \tau}{\partial \xi}\left(\xi_{N}\right)\right)$ and $\mathbf{h}=\left[h_{0}, h_{1}, \cdots, h_{N}\right]^{T}$. Among these two parts, $\partial \tau / \partial \xi$ can be determined with

$$
\frac{\partial \tau}{\partial \xi}=\sqrt{\left(\frac{\partial x}{\partial \xi}\right)^{2}+\left(\frac{\partial y}{\partial \xi}\right)^{2}}
$$

\section{A.2.3 Discretization in a 2D quadrilateral element}

For a 2D quadrilateral spectral-element, a similar method mentioned in subsection A.2.2 has been used for mapping $u(x, y)$. A continuous base function $u\left(\xi_{1}, \xi_{2}\right) \in$ $[-1,1] \times[-1,1]$ can be expressed as follows

$$
u\left(\xi_{1}, \xi_{2}\right)=\sum_{i=0}^{N} \sum_{j=0}^{N} u_{i j} \Psi_{i}\left(\xi_{1}\right) \Psi_{j}\left(\xi_{2}\right)
$$

where $\Psi_{i}(\xi)$ are the nodal base functions using GLL interpolants. And then, the spatial derivatives of $u\left(\xi_{1}, \xi_{2}\right)$ can be derived as

$$
\frac{\partial u\left(\xi_{1}, \xi_{2}\right)}{\partial \xi_{1}}=\sum_{i=0}^{N} \sum_{j=0}^{N} u_{i j} \frac{\partial \Psi_{i}\left(\xi_{1}\right)}{\partial \xi_{1}} \Psi_{j}\left(\xi_{2}\right)=\hat{\mathbf{D}} \otimes \hat{\mathbf{I}} \mathbf{u}=\mathbf{D}_{1} \mathbf{u}
$$




$$
\frac{\partial u\left(\xi_{1}, \xi_{2}\right)}{\partial \xi_{2}}=\sum_{i=0}^{N} \sum_{j=0}^{N} u_{i j} \Psi_{i}\left(\xi_{1}\right) \frac{\partial \Psi_{j}\left(\xi_{2}\right)}{\partial \xi_{2}}=\hat{\mathbf{I}} \otimes \hat{\mathbf{D}} \mathbf{u}=\mathbf{D}_{2} \mathbf{u}
$$

Here, the symbol $\otimes$ represents the Kronecker product operator. $\mathbf{u}$ is defined as $\mathbf{u}=\left[u_{00}, u_{10}, \cdots, u_{N 0}, u_{01}, u_{11}, \cdots, u_{N N}\right]^{T}$.

In order to determine the spatial derivatives of a function $u(x, y)$, the employed chain rule is presented as

$$
\begin{gathered}
\frac{\partial}{\partial x}=\frac{\partial \xi_{1}}{\partial x} \frac{\partial}{\partial \xi_{1}}+\frac{\partial \xi_{2}}{\partial x} \frac{\partial}{\partial \xi_{2}} \\
\frac{\partial}{\partial y}=\frac{\partial \xi_{1}}{\partial y} \frac{\partial}{\partial \xi_{1}}+\frac{\partial \xi_{2}}{\partial y} \frac{\partial}{\partial \xi_{2}}
\end{gathered}
$$

Meanwhile, the transformation tensor can be expressed as

$$
\left[\begin{array}{cc}
\frac{\partial \xi_{1}}{\partial x} & \frac{\partial \xi_{1}}{\partial y} \\
\frac{\partial \xi_{2}}{\partial x} & \frac{\partial \xi_{2}}{\partial y}
\end{array}\right]=\left[\begin{array}{cc}
\frac{\partial x}{\partial \xi_{1}} & \frac{\partial x}{\partial \xi_{2}} \\
\frac{\partial y}{\partial \xi_{1}} & \frac{\partial y}{\partial \xi_{2}}
\end{array}\right]^{-1}=\frac{1}{J}\left[\begin{array}{cc}
\frac{\partial y}{\partial \xi_{2}} & \frac{\partial y}{\partial \xi_{1}} \\
\frac{\partial x}{\partial \xi_{2}} & \frac{\partial x}{\partial \xi_{1}}
\end{array}\right]
$$

where the transformation Jacobian is defined as $J=\partial x / \partial \xi_{1} \partial y / \partial \xi_{2}-\partial x / \partial \xi_{2} \partial y / \partial \xi_{1}$. Therefore, the spatial derivatives as a function of $x$ and $y$ read

$$
\begin{aligned}
& \mathbf{D}_{x}=\Lambda\left(\frac{\partial \xi_{1}}{\partial x}\right) \mathbf{D}_{1}+\Lambda\left(\frac{\partial \xi_{2}}{\partial x}\right) \mathbf{D}_{2}, \\
& \mathbf{D}_{y}=\Lambda\left(\frac{\partial \xi_{1}}{\partial y}\right) \mathbf{D}_{1}+\Lambda\left(\frac{\partial \xi_{2}}{\partial y}\right) \mathbf{D}_{2},
\end{aligned}
$$

where function $\Lambda\left(f_{i j}\right)$ returns a matrix of rank $(N+1)^{2}$ with the diagonal components being the vector $f_{i j}$.

By employing the transformation Jacobian $J$, the integration in a 2D quadrilateral 
element can be transformed into the canonical domain $\Omega_{s t} \in[-1,1] \times[-1,1]$ as follows

$$
\begin{aligned}
& \int_{\Omega} u(x, y) d x d y=\int_{\Omega_{s t}} u\left(\xi_{1}, \xi_{2}\right) J d \xi_{1} d \xi_{2} \\
& =\sum_{i=0}^{N} \sum_{j=0}^{N} u_{i j} \int_{-1}^{1} \int_{-1}^{1} J\left(\xi_{1}, \xi_{2}\right) \Psi_{i}\left(\xi_{1}\right) \Psi_{j}\left(\xi_{2}\right) d \xi_{1} d \xi_{2} \\
& =\sum_{i=0}^{N} \sum_{j=0}^{N} u_{i j} \sum_{p=0}^{N} w_{p} \int_{-1}^{1} J\left(\xi_{1 p}, \xi_{2}\right) \Psi_{i}\left(\xi_{1 p}\right) \Psi_{j}\left(\xi_{2}\right) d \xi_{2} \\
& =\sum_{i=0}^{N} \sum_{j=0}^{N} u_{i j} \sum_{p=0}^{N} w_{p} \sum_{q=0}^{N} w_{q} J\left(\xi_{1 p}, \xi_{2 q}\right) \Psi_{i}\left(\xi_{1 p}\right) \Psi_{j}\left(\xi_{2 q}\right) \\
& =\sum_{i=0}^{N} \sum_{j=0}^{N} u_{i j} \sum_{p=0}^{N} w_{p} \sum_{q=0}^{N} w_{q} J\left(\xi_{1 p}, \xi_{2 q}\right) \delta_{i p} \delta_{j q} \\
& =\sum_{i=0}^{N} \sum_{j=0}^{N} u_{i j} w_{i} w_{j} J_{i j} \\
& =\mathbf{w}^{T} \mathbf{u}
\end{aligned}
$$

where $\mathbf{w}=\left[w_{0} w_{0} J_{00}, w_{1} w_{0} J_{10}, \cdots, w_{N} w_{N} J_{N N}\right]^{T}$.

The weight functions in a 2D quadrilateral element are also defined with the nodal base functions, which is as same as the method in subsection A.2.2, i.e.,

$$
v_{m n}=\Psi_{m}\left(\xi_{1}\right) \Psi_{n}\left(\xi_{2}\right)
$$

where $m, n=0,1,2, \cdots N$. As such,

$$
\begin{aligned}
\left(u, v_{m n}\right) & =\sum_{i=0}^{N} \sum_{j=0}^{N} u_{i j} \int_{-1}^{1} \int_{-1}^{1} J\left(\xi_{1}, \xi_{2}\right) \Psi_{i}\left(\xi_{1}\right) \Psi_{j}\left(\xi_{2}\right) \Psi_{m}\left(\xi_{1}\right) \Psi_{n}\left(\xi_{2}\right) d \xi_{1} d \xi_{2} \\
& =\sum_{i=0}^{N} \sum_{j=0}^{N} u_{i j} \sum_{p=0}^{N} \sum_{q=0}^{N} J\left(\xi_{1 p}, \xi_{2 q}\right) w_{p} w_{q} \delta_{i p} \delta_{m p} \delta_{j q} \delta_{n q} \\
& =\mathbf{v}_{p q}^{T} \mathbf{W u} .
\end{aligned}
$$

Here, $\mathbf{W}=\mathbf{I} \mathbf{w}$ where $\mathbf{I}$ is the identical matrix of $\operatorname{rank}(N+1)^{2}$. The value in vector $\mathbf{v}_{p q}$ is one at the $p q$-th component and zero at any other components. Therefore, for all weight functions in a $2 \mathrm{D}$ quadrilateral element, the above expression can be further expanded into a matrix form as follows

$$
(u, v)=\mathbf{W u}
$$


The discretization of $(\nabla u, \nabla v)$ in a 2D quadrilateral element for Eq. (A.18) can be generally written as

$$
\begin{aligned}
(\nabla u, \nabla v) & =\left(\frac{\partial u}{\partial x}, \frac{\partial v}{\partial x}\right)+\left(\frac{\partial u}{\partial y}, \frac{\partial v}{\partial y}\right) \\
& =\left(\mathbf{D}_{x} \mathbf{u}, \mathbf{D}_{x} \mathbf{v}\right)+\left(\mathbf{D}_{y} \mathbf{u}, \mathbf{D}_{y} \mathbf{v}\right) \\
& =\mathbf{D}_{x}^{T} \mathbf{W} \mathbf{D}_{x} \mathbf{u}+\mathbf{D}_{y}^{T} \mathbf{W} \mathbf{D}_{y} \mathbf{u} \\
& =\left(\mathbf{D}_{x}^{T} \mathbf{W} \mathbf{D}_{x}+\mathbf{D}_{y}^{T} \mathbf{W} \mathbf{D}_{y}\right) \mathbf{u}
\end{aligned}
$$

Therefore, the local elemental linear operator (the first two-components of Eq. (A.18)) can be presented as

$$
\int_{\Omega_{e}} \nabla u \nabla v d \Omega_{e}+\lambda^{2} \int_{\Omega_{e}} u v d \Omega_{e}=\left(\mathbf{D}_{x}^{T} \mathbf{W} \mathbf{D}_{x}+\mathbf{D}_{y}^{T} \mathbf{W} \mathbf{D}_{y}+\lambda \mathbf{W}\right) \mathbf{u} \equiv \mathbf{A}_{e} \mathbf{u}
$$

where $\mathbf{D}_{x}^{T} \mathbf{W D}_{x}+\mathbf{D}_{y}^{T} \mathbf{W} \mathbf{D}_{y}$ and $\mathbf{W}$ represent stiffness and mass matrices, respectively.

\section{A.2.4 Global operations}

In order to express the local $\mathbf{u}_{l}$ using the global degree of freedom $\mathbf{u}_{g}$, an assembly matrix $\mathbf{G}$ has to be introduced, which reads

$$
\mathbf{u}_{l}=\mathbf{G u}_{g}
$$

Therefore, each component of Eq. (A.17) can be discretized as

$$
\begin{gathered}
\int_{\Omega} \nabla u \nabla v d \Omega+\lambda^{2} \int_{\Omega} u v d \Omega=\mathbf{G}^{T}\left(\mathbf{A}_{e} \otimes \mathbf{I}_{e}\right) \mathbf{G u}_{g} \\
-\int_{\Omega} f v d \Omega=\mathbf{G}^{T}\left(\mathbf{W}_{e} \otimes \mathbf{I}_{e}\right) \mathbf{f}_{e}
\end{gathered}
$$


and

$$
\oint_{\partial \Omega} h v d(\partial \Omega)=\mathbf{G}^{T}\left(\mathbf{B}_{e} \otimes \mathbf{I}_{e}\right) \mathbf{h}_{e}
$$

respectively, where $\mathbf{I}_{e}$ is an identical matrix of rank as same as the total element number. Therefore, Eq. (A.17) becomes

$$
\mathbf{G}^{T}\left(\mathbf{A}_{e} \otimes \mathbf{I}_{e}\right) \mathbf{G} \mathbf{u}_{g}=\mathbf{G}^{T}\left(\mathbf{W}_{e} \otimes \mathbf{I}_{e}\right) \mathbf{f}_{e}+\mathbf{G}^{T}\left(\mathbf{B}_{e} \otimes \mathbf{I}_{e}\right) \mathbf{h}_{e}
$$

then it can be expressed as $\mathbf{A}_{g} \mathbf{u}_{g}=\mathbf{b}_{g}$.

The final expression can also be further presented employing the Dirichlet boundary condition, $\mathbf{u}_{g}=\left[\mathbf{u}_{g}^{u}, \mathbf{u}_{g}^{D}\right]^{T}$, where the superscripts $(\cdot)^{u}$ and $(\cdot)^{D}$ are the unknown and Dirichlet degree of freedom (DOF), respectively, as follows

$$
\left[\begin{array}{cc}
\mathbf{A} & \mathbf{A}^{D} \\
0 & \mathbf{I}^{D}
\end{array}\right]\left[\begin{array}{c}
\mathbf{u}_{g}^{u} \\
\mathbf{u}_{g}^{D}
\end{array}\right]=\left[\begin{array}{c}
\mathbf{b}_{g}^{u} \\
\mathbf{b}_{g}^{D}
\end{array}\right] .
$$

Therefore, the linear equation must be solved with

$$
\mathbf{A} \mathbf{u}_{g}^{u}=\mathbf{b}_{g}^{u}-\mathbf{A}^{D} \mathbf{u}_{g}^{D} \equiv \mathbf{b}
$$

\section{A.3 Linear solver}

The above 2D Helmholtz equation (Eq. (A.50)) needs to be employed to solve both the pressure and viscous substeps at each wavenumber. Particularly, for the viscous substep, a large value of $1 /(\nu \Delta t)$ results in a strong domination on the diagonal components of the matrix $\mathbf{A}$, indicating that a Jacobian preconditioned-conjugategradient $(\mathrm{PCG})$ solver is enough for solving the equation. In contrast, for the pressure substep, due to a poor Jacobian-preconditioned effect on the matrix $\mathbf{A}$, the $2 \mathrm{D}$ 
Helmholtz equation has to be calculated by a multigrid solver. It is noticed that Semtex allows the parallel computation in the homogeneous (or Fourier) direction, and each 2D Helmholtz equation has to be solved in a sequence. A direct solver is possibly required to avoid the complex multigrid implementation in the pressure substep.

\section{A.3.1 Iterative solver for the viscous substep}

In order to solve $2 \mathrm{D}$ Helmholtz equation for the viscous substep, such as $\mathbf{A} \mathbf{u}^{u}=\mathbf{b}$, a basic iterative solver can be presented as follows

$$
\mathbf{u}_{k+1}^{u}=\mathbf{u}_{k}^{u}+\mathbf{P}^{-1} r_{n} \quad \text { for } k=0,1,2, \cdots,
$$

where $\mathbf{P}$ is a preconditioning matrix for relaxation, and $r_{n}$ is a residual matrix defined as $r_{n}=\mathbf{b}-\mathbf{A} \mathbf{u}_{k}^{u}$. To avoid a slow convergence rate, $\mathbf{P}$ needs to satisfy a requirement that it has to be close to $\mathbf{A}$ as much as possible. Therefore, a Jacobian preconditioner is introduced in the viscous substep, i.e. $\mathbf{P} \equiv \operatorname{diag}(\mathbf{A})$.

The PCG solver begins with an initial guess $\mathbf{u}_{0}^{u}$ and performs the following iterating steps: 


$$
\begin{aligned}
& r_{0}=\mathbf{b}-\mathbf{A} \mathbf{u}_{0}^{u}, z_{0}=\mathbf{P}^{-1} r_{0} \\
& w_{0}=z_{0} \text { and } j=0
\end{aligned}
$$

Loop $j$ :

$$
\begin{aligned}
\alpha_{j} & =\left(r_{j}, z_{j}\right) /\left(\mathbf{A} w_{j}, w_{j}\right) \\
\mathbf{u}_{j+1}^{u} & =\mathbf{u}_{j}^{u}+\alpha_{j} w_{j} \\
r_{j+1} & =r_{j}-\alpha_{j} \mathbf{A} w_{j} \\
z_{j+1} & =\mathbf{P}^{-1} r_{j+1} \\
\beta_{j} & =\left(r_{j+1}, z_{j+1}\right) /\left(r_{j}, z_{j}\right) \\
w_{j+1} & =z_{j+1}+\beta_{j} w_{j}
\end{aligned}
$$

break if converged

\section{End}

In fact, $\mathbf{A} w_{j}$ is solved employing a local elemental matrix $\mathbf{A}_{e}$ and globally assembled afterward in the PCG solver.

\section{A.3.2 Direct solver for the pressure substep}

For the pressure substep, a direct solver can avoid a complication in use of multigrid solver. However, the inverse matrix $\mathbf{A}^{-1}$ from direct solver results in a huge cost on computational resources. Therefore, a so-called static condensation technique is involved in order to avoid this situation. The function of the static condensation is to split $\mathbf{u}^{u}$ into element-interior DOF $\mathbf{u}_{i}^{u}$ and element-boundary DOF $\mathbf{u}_{b}^{u}$. Therefore, $\mathbf{A} \mathbf{u}^{u}=\mathbf{b}$ can be expressed as follows

$$
\left[\begin{array}{ll}
\mathbf{A}_{b} & \mathbf{A}_{c} \\
\mathbf{A}_{c}^{T} & \mathbf{A}_{i}
\end{array}\right]\left[\begin{array}{l}
\mathbf{u}_{b}^{u} \\
\mathbf{u}_{i}^{u}
\end{array}\right]=\left[\begin{array}{l}
\mathbf{b}_{b} \\
\mathbf{b}_{i}
\end{array}\right]
$$


By simple manipulation, the above expression can be further written as

$$
\left[\begin{array}{cc}
\mathbf{A}_{b}-\mathbf{A}_{c} \mathbf{A}_{i}^{-1} \mathbf{A}_{c}^{T} & 0 \\
\mathbf{A}_{c}^{T} & \mathbf{A}_{i}
\end{array}\right]\left[\begin{array}{c}
\mathbf{u}_{b}^{u} \\
\mathbf{u}_{i}^{u}
\end{array}\right]=\left[\begin{array}{c}
\mathbf{b}_{b}-\mathbf{A}_{c} \mathbf{A}_{i}^{-1} \mathbf{b}_{i} \\
\mathbf{b}_{i}
\end{array}\right]
$$

From above equation, it is straightforward to determine boundary unknowns with

$$
\mathbf{u}_{b}^{u}=\left(\mathbf{A}_{b}-\mathbf{A}_{c} \mathbf{A}_{i}^{-1} \mathbf{A}_{c}^{T}\right)^{-1}\left(\mathbf{b}_{b}-\mathbf{A}_{c} \mathbf{A}_{i}^{-1} \mathbf{b}_{i}\right)
$$

Here, the rank of $\left(\mathbf{A}_{b}-\mathbf{A}_{c} \mathbf{A}_{i}^{-1} \mathbf{A}_{c}^{T}\right)^{-1}$ is significantly lower than it of $\mathbf{A}^{-1}$, indicating that the usage of memory for the element-boundary matrix is not much expensive. Moreover, the interior unknowns can be determine directly by

$$
\mathbf{u}_{i}^{u}=\mathbf{A}_{i}^{-1} \mathbf{b}_{i}-\mathbf{A}_{i}^{-1} \mathbf{A}_{c}^{T} \mathbf{u}_{b}^{u}
$$




\section{Appendix B}

\section{Transport equations of Reynolds}

\section{stresses}

The transport equations of Reynolds stresses $\left\langle u_{i}^{\prime} u_{j}^{\prime}\right\rangle$ in a radially-rotating reference frame can be expressed as

$$
H_{i j}-P_{i j}-\Pi_{i j}+\varepsilon_{i j}-D_{i j}-C_{i j}=0
$$

where $H_{i j}, P_{i j}, \Pi_{i j}, \varepsilon_{i j}, D_{i j}$ and $C_{i j}$ represent the convection, production, pressurestrain, viscous dissipation, diffusion (consisting of turbulent, pressure and viscous diffusion effects) and Coriolis production terms, respectively.

In this appendix, only the transport equations of four non-trivial Reynolds stresses are provided in a cylindrical coordinate system, which are $\left\langle u_{z}^{\prime} u_{z}^{\prime}\right\rangle,\left\langle u_{r}^{\prime} u_{r}^{\prime}\right\rangle,\left\langle u_{z}^{\prime} u_{r}^{\prime}\right\rangle$ and $\left\langle u_{\theta}^{\prime} u_{\theta}^{\prime}\right\rangle$. Based on a literature study, it is concluded that there is a necessity to present these transport equations here, as the exact forms of these equations are not readily available in the literature. 
The transport equation of $\left\langle u_{z}^{\prime} u_{z}^{\prime}\right\rangle$ reads

$$
\begin{aligned}
& \underbrace{\left\langle u_{r}\right\rangle \frac{\partial\left\langle u_{z}^{\prime} u_{z}^{\prime}\right\rangle}{\partial r}+\frac{\left\langle u_{\theta}\right\rangle}{r} \frac{\partial\left\langle u_{z}^{\prime} u_{z}^{\prime}\right\rangle}{\partial \theta}+\left\langle u_{z}\right\rangle \frac{\partial\left\langle u_{z}^{\prime} u_{z}^{\prime}\right\rangle}{\partial z}}_{H_{z z}} \\
& -(\underbrace{-2\left\langle u_{r}^{\prime} u_{z}^{\prime}\right\rangle \frac{\partial\left\langle u_{z}\right\rangle}{\partial r}-2 \frac{\left\langle u_{\theta}^{\prime} u_{z}^{\prime}\right\rangle}{r} \frac{\partial\left\langle u_{z}\right\rangle}{\partial \theta}-2\left\langle u_{z}^{\prime} u_{z}^{\prime}\right\rangle \frac{\partial\left\langle u_{z}\right\rangle}{\partial z}}_{P_{z z}})-\underbrace{\frac{2}{\rho}\left\langle p^{\prime} \frac{\partial u_{z}^{\prime}}{\partial z}\right\rangle}_{\Pi_{z z}} \\
& +\underbrace{2 \nu\left[\left\langle\frac{\partial u_{z}^{\prime}}{\partial r} \frac{\partial u_{z}^{\prime}}{\partial r}\right\rangle+\frac{1}{r^{2}}\left\langle\frac{\partial u_{z}^{\prime}}{\partial \theta} \frac{\partial u_{z}^{\prime}}{\partial \theta}\right\rangle+\left\langle\frac{\partial u_{z}^{\prime}}{\partial z} \frac{\partial u_{z}^{\prime}}{\partial z}\right\rangle\right]}_{\varepsilon_{z z}} \\
& -\{\begin{array}{l}
-\frac{1}{r} \frac{\partial r\left\langle u_{r}^{\prime} u_{z}^{\prime} u_{z}^{\prime}\right\rangle}{\partial r}-\frac{1}{r} \frac{\partial\left\langle u_{\theta}^{\prime} u_{z}^{\prime} u_{z}^{\prime}\right\rangle}{\partial \theta}-\frac{\partial\left\langle u_{z}^{\prime} u_{z}^{\prime} u_{z}^{\prime}\right\rangle}{\partial z}-\frac{2}{\rho} \frac{\partial\left\langle u_{z}^{\prime} p^{\prime}\right\rangle}{\partial z} \\
+\nu\left[\frac{1}{r} \frac{\partial}{\partial r}\left(r \frac{\partial\left\langle u_{z}^{\prime} u_{z}^{\prime}\right\rangle}{\partial r}\right)+\frac{1}{r^{2}} \frac{\partial^{2}\left\langle u_{z}^{\prime} u_{z}^{\prime}\right\rangle}{\partial \theta^{2}}+\frac{\partial^{2}\left\langle u_{z}^{\prime} u_{z}^{\prime}\right\rangle}{\partial z^{2}}\right]
\end{array} \underbrace{}_{D_{z z}}\} \\
& -[\underbrace{-4 \Omega\left(\left\langle u_{r}^{\prime} u_{z}^{\prime}\right\rangle \sin \theta+\left\langle u_{\theta}^{\prime} u_{z}^{\prime}\right\rangle \cos \theta\right)}_{C_{z z}}]=0 \text {. }
\end{aligned}
$$


The transport equation of $\left\langle u_{r}^{\prime} u_{r}^{\prime}\right\rangle$ is

$$
\begin{aligned}
& \underbrace{\left\langle u_{r}\right\rangle \frac{\partial\left\langle u_{r}^{\prime} u_{r}^{\prime}\right\rangle}{\partial r}+\frac{\left\langle u_{\theta}\right\rangle}{r} \frac{\partial\left\langle u_{r}^{\prime} u_{r}^{\prime}\right\rangle}{\partial \theta}+\left\langle u_{z}\right\rangle \frac{\partial\left\langle u_{r}^{\prime} u_{r}^{\prime}\right\rangle}{\partial z}-2\left\langle u_{\theta}\right\rangle \frac{\left\langle u_{r}^{\prime} u_{\theta}^{\prime}\right\rangle}{r}}_{H_{r r}} \\
& -(\underbrace{2\left\langle u_{r}^{\prime} u_{\theta}^{\prime}\right\rangle \frac{\left\langle u_{\theta}\right\rangle}{r}-2\left\langle u_{r}^{\prime} u_{r}^{\prime}\right\rangle \frac{\partial\left\langle u_{r}\right\rangle}{\partial r}-2 \frac{\left\langle u_{r}^{\prime} u_{\theta}^{\prime}\right\rangle}{r} \frac{\partial\left\langle u_{r}\right\rangle}{\partial \theta}-2\left\langle u_{r}^{\prime} u_{z}^{\prime}\right\rangle \frac{\partial\left\langle u_{r}\right\rangle}{\partial z}}_{P_{r r}})-\underbrace{\frac{2}{\rho}\left\langle p^{\prime} \frac{\partial u_{r}^{\prime}}{\partial r}\right\rangle}_{\Pi_{r r}} \\
& +\underbrace{2 \nu\left[\left\langle\frac{\partial u_{r}^{\prime}}{\partial r} \frac{\partial u_{r}^{\prime}}{\partial r}\right\rangle+\frac{1}{r^{2}}\left\langle\left(\frac{\partial u_{r}^{\prime}}{\partial \theta}-u_{\theta}^{\prime}\right)^{2}\right\rangle+\left\langle\frac{\partial u_{r}^{\prime}}{\partial z} \frac{\partial u_{r}^{\prime}}{\partial z}\right\rangle-\frac{2}{r^{2}} \frac{\partial\left\langle u_{r}^{\prime} u_{\theta}^{\prime}\right\rangle}{\partial \theta}\right]}_{\varepsilon_{r r}} \\
& -\left\{\begin{array}{l}
2 \frac{\left\langle u_{r}^{\prime} u_{\theta}^{\prime} u_{\theta}^{\prime}\right\rangle}{r}-\frac{1}{r} \frac{\partial r\left\langle u_{r}^{\prime} u_{r}^{\prime} u_{r}^{\prime}\right\rangle}{\partial r}-\frac{1}{r} \frac{\partial\left\langle u_{r}^{\prime} u_{r}^{\prime} u_{\theta}^{\prime}\right\rangle}{\partial \theta}-\frac{\partial\left\langle u_{r}^{\prime} u_{r}^{\prime} u_{z}^{\prime}\right\rangle}{\partial z}-\frac{2}{\rho} \frac{\partial\left\langle u_{r}^{\prime} p^{\prime}\right\rangle}{\partial r} \\
+\nu\left[\frac{1}{r} \frac{\partial}{\partial r}\left(r \frac{\partial\left\langle u_{r}^{\prime} u_{r}^{\prime}\right\rangle}{\partial r}\right)+\frac{1}{r^{2}} \frac{\partial^{2}\left\langle u_{r}^{\prime} u_{r}^{\prime}\right\rangle}{\partial \theta^{2}}-\frac{2}{r^{2}}\left(\left\langle u_{r}^{\prime} u_{r}^{\prime}\right\rangle-\left\langle u_{\theta}^{\prime} u_{\theta}^{\prime}\right\rangle\right)+\frac{\partial^{2}\left\langle u_{r}^{\prime} u_{r}^{\prime}\right\rangle}{\partial z^{2}}\right]
\end{array}\right\} \\
& -(\underbrace{4 \Omega\left\langle u_{r}^{\prime} u_{z}^{\prime}\right\rangle \sin \theta}_{C_{r r}})=0 \text {. }
\end{aligned}
$$


The transport equation of $\left\langle u_{z}^{\prime} u_{r}^{\prime}\right\rangle$ reads

$$
\begin{aligned}
& \underbrace{\left\langle u_{r}\right\rangle \frac{\partial\left\langle u_{r}^{\prime} u_{z}^{\prime}\right\rangle}{\partial r}+\frac{\left\langle u_{\theta}\right\rangle}{r} \frac{\partial\left\langle u_{r}^{\prime} u_{z}^{\prime}\right\rangle}{\partial \theta}+\left\langle u_{z}\right\rangle \frac{\partial\left\langle u_{r}^{\prime} u_{z}^{\prime}\right\rangle}{\partial z}-\left\langle u_{\theta}\right\rangle \frac{\left\langle u_{\theta}^{\prime} u_{z}^{\prime}\right\rangle}{r}}_{H_{z r}} \\
& -\left(\begin{array}{l}
\left\langle u_{\theta}^{\prime} u_{z}^{\prime}\right\rangle \frac{\left\langle u_{\theta}\right\rangle}{r}-\left\langle u_{r}^{\prime} u_{z}^{\prime}\right\rangle \frac{\partial\left\langle u_{r}\right\rangle}{\partial r}-\left\langle u_{r}^{\prime} u_{r}^{\prime}\right\rangle \frac{\partial\left\langle u_{z}\right\rangle}{\partial r}-\frac{\left\langle u_{\theta}^{\prime} u_{z}^{\prime}\right\rangle}{r} \frac{\partial\left\langle u_{r}\right\rangle}{\partial \theta} \\
-\frac{\left\langle u_{r}^{\prime} u_{\theta}^{\prime}\right\rangle}{r} \frac{\left.\partial u_{z}\right\rangle}{\partial \theta}-\left\langle u_{z}^{\prime} u_{z}^{\prime}\right\rangle \frac{\partial\left\langle u_{r}\right\rangle}{\partial z}-\left\langle u_{r}^{\prime} u_{z}^{\prime}\right\rangle \frac{\partial\left\langle u_{z}\right\rangle}{\partial z}
\end{array}\right) \\
& -\underbrace{\frac{1}{\rho}\left(\left\langle p^{\prime} \frac{\partial u_{r}^{\prime}}{\partial z}\right\rangle+\left\langle p^{\prime} \frac{\partial u_{z}^{\prime}}{\partial r}\right\rangle\right)}_{\Pi_{z r}} \\
& +\underbrace{2 \nu\left[\left\langle\frac{\partial u_{r}^{\prime}}{\partial r} \frac{\partial u_{z}^{\prime}}{\partial r}\right\rangle+\frac{1}{r^{2}}\left\langle\left(\frac{\partial u_{r}^{\prime}}{\partial \theta}-u_{\theta}^{\prime}\right) \frac{\partial u_{z}^{\prime}}{\partial \theta}\right\rangle+\left\langle\frac{\partial u_{r}^{\prime}}{\partial z} \frac{\partial u_{z}^{\prime}}{\partial z}\right\rangle+\frac{1}{r^{2}} \frac{\partial\left\langle u_{\theta}^{\prime} u_{z}^{\prime}\right\rangle}{\partial \theta}\right]}_{\varepsilon_{z r}} \\
& -\left\{\begin{array}{l}
\frac{\left\langle u_{\theta}^{\prime} u_{\theta}^{\prime} u_{z}^{\prime}\right\rangle}{r}-\frac{1}{r} \frac{\partial r\left\langle u_{r}^{\prime} u_{r}^{\prime} u_{z}^{\prime}\right\rangle}{\partial r}-\frac{1}{r} \frac{\partial\left\langle u_{r}^{\prime} u_{\theta}^{\prime} u_{z}^{\prime}\right\rangle}{\partial \theta}-\frac{\partial\left\langle u_{r}^{\prime} u_{z}^{\prime} u_{z}^{\prime}\right\rangle}{\partial z} \\
-\frac{1}{\rho}\left(\frac{\partial\left\langle u_{z}^{\prime} p^{\prime}\right\rangle}{\partial r}+\frac{\partial\left\langle u_{r}^{\prime} p^{\prime}\right\rangle}{\partial z}\right) \\
\underbrace{+\nu\left[\frac{1}{r} \frac{\partial}{\partial r}\left(r \frac{\partial\left\langle u_{r}^{\prime} u_{z}^{\prime}\right\rangle}{\partial r}\right)+\frac{1}{r^{2}} \frac{\partial^{2}\left\langle u_{r}^{\prime} u_{z}^{\prime}\right\rangle}{\partial \theta^{2}}-\frac{\left\langle u_{r}^{\prime} u_{z}^{\prime}\right\rangle}{r^{2}}+\frac{\partial^{2}\left\langle u_{r}^{\prime} u_{z}^{\prime}\right\rangle}{\partial z^{2}}\right]}_{D_{z r}}
\end{array}\right\} \\
& -[\underbrace{2 \Omega\left(\left\langle u_{z}^{\prime} u_{z}^{\prime}\right\rangle \sin \theta-\left\langle u_{r}^{\prime} u_{r}^{\prime}\right\rangle \sin \theta-\left\langle u_{r}^{\prime} u_{\theta}^{\prime}\right\rangle \cos \theta\right)}_{C_{z r}}]=0 \text {. }
\end{aligned}
$$


The transport equation of $\left\langle u_{\theta}^{\prime} u_{\theta}^{\prime}\right\rangle$ is

$$
\begin{aligned}
& \underbrace{\left\langle u_{r}\right\rangle \frac{\partial\left\langle u_{\theta}^{\prime} u_{\theta}^{\prime}\right\rangle}{\partial r}+\frac{\left\langle u_{\theta}\right\rangle}{r} \frac{\partial\left\langle u_{\theta}^{\prime} u_{\theta}^{\prime}\right\rangle}{\partial \theta}+\left\langle u_{z}\right\rangle \frac{\partial\left\langle u_{\theta}^{\prime} u_{\theta}^{\prime}\right\rangle}{\partial z}+2\left\langle u_{\theta}\right\rangle \frac{\left\langle u_{\theta}^{\prime} u_{\theta}^{\prime}\right\rangle}{r}}_{H_{\theta \theta}} \\
& -(\underbrace{-2\left\langle u_{r}^{\prime} u_{\theta}^{\prime}\right\rangle \frac{\left\langle u_{\theta}\right\rangle}{r}-2\left\langle u_{r}^{\prime} u_{\theta}^{\prime}\right\rangle \frac{\partial\left\langle u_{\theta}\right\rangle}{\partial r}-2 \frac{\left\langle u_{\theta}^{\prime} u_{\theta}^{\prime}\right\rangle}{r} \frac{\partial\left\langle u_{\theta}\right\rangle}{\partial \theta}-2\left\langle u_{\theta}^{\prime} u_{z}^{\prime}\right\rangle \frac{\partial\left\langle u_{\theta}\right\rangle}{\partial z}}_{P_{\theta \theta}}) \\
& -\underbrace{\frac{2}{\rho r}\left\langle p^{\prime} \frac{\partial u_{\theta}^{\prime}}{\partial \theta}\right\rangle}_{\Pi_{\theta \theta}} \\
& +\underbrace{2 \nu\left[\left\langle\frac{\partial u_{\theta}^{\prime}}{\partial r} \frac{\partial u_{\theta}^{\prime}}{\partial r}\right\rangle+\frac{1}{r^{2}}\left\langle\left(\frac{\partial u_{\theta}^{\prime}}{\partial \theta}+u_{r}^{\prime}\right)^{2}\right\rangle+\left\langle\frac{\partial u_{\theta}^{\prime}}{\partial z} \frac{\partial u_{\theta}^{\prime}}{\partial z}\right\rangle-\frac{2}{r^{2}} \frac{\partial\left\langle u_{r}^{\prime} u_{\theta}^{\prime}\right\rangle}{\partial \theta}\right]}_{\varepsilon_{\theta \theta}} \\
& -\left\{\begin{array}{l}
-2 \frac{\left\langle u_{r}^{\prime} u_{\theta}^{\prime} u_{\theta}^{\prime}\right\rangle}{r}-\frac{1}{r} \frac{\partial r\left\langle u_{r}^{\prime} u_{\theta}^{\prime} u_{\theta}^{\prime}\right\rangle}{\partial r}-\frac{1}{r} \frac{\partial\left\langle u_{\theta}^{\prime} u_{\theta}^{\prime} u_{\theta}^{\prime}\right\rangle}{\partial \theta}-\frac{\partial\left\langle u_{\theta}^{\prime} u_{\theta}^{\prime} u_{z}^{\prime}\right\rangle}{\partial z}-\frac{2}{\rho r} \frac{\partial\left\langle u_{\theta}^{\prime} p^{\prime}\right\rangle}{\partial \theta} \\
+\nu\left[\frac{1}{r} \frac{\partial}{\partial r}\left(r \frac{\partial\left\langle u_{\theta}^{\prime} u_{\theta}^{\prime}\right\rangle}{\partial r}\right)+\frac{1}{r^{2}} \frac{\partial^{2}\left\langle u_{\theta}^{\prime} u_{\theta}^{\prime}\right\rangle}{\partial \theta^{2}}+\frac{2}{r^{2}}\left(\left\langle u_{r}^{\prime} u_{r}^{\prime}\right\rangle-\left\langle u_{\theta}^{\prime} u_{\theta}^{\prime}\right\rangle\right)+\frac{\partial^{2}\left\langle u_{\theta}^{\prime} u_{\theta}^{\prime}\right\rangle}{\partial z^{2}}\right]
\end{array}\right\} \\
& -(\underbrace{4 \Omega\left\langle u_{\theta}^{\prime} u_{z}^{\prime}\right\rangle \cos \theta}_{C_{\theta \theta}})=0 \text {. }
\end{aligned}
$$

